EFFECTS OF CLIMATE VARIABILITY AND HUMAN ACTIVITY ON TERRESTRIAL WATER STORAGE CHANGES AT BASIN SCALE: A CASE STUDY OF THE YANGTZE RIVER BASIN 


\section{Promotion Committee:}

Chairman: $\quad$ Prof. dr. G. P. M. R. Dewulf

Secretary: $\quad$ Prof. dr. G. P. M. R. Dewulf University of Twente

Supervisors: Prof. dr. ir. A. Y. Hoekstra University of Twente Prof. dr. Z. Su University of Twente

Co-supervisor: Dr. ir. M. S. Salama University of Twente

Referee: Dr. M.S. Krol University of Twente

Examining

Committee: Prof. dr. ir. J. C. J. Kwadijk University of Twente Prof. ir. E. van Beek University of Twente Prof. dr. Y. Zhou East China Normal University

Prof. dr. M. Mancini Politecnico di Milano

ISBN: 978-94-6233-207-2

Copyright (C) 2015 by Y. Huang

No part of this work may be reproduced by print, photocopy or any other means without prior written permission of the author.

DOI: $10.3990 / 1.9789462332072$

URL: http://dx.doi.org/10.3990/1.9789462332072

Printed by Gildeprint Drukkerijen, Enschede, the Netherlands 


\title{
EFFECTS OF CLIMATE VARIABILITY AND HUMAN ACTIVITY ON TERRESTRIAL WATER STORAGE CHANGES AT BASIN SCALE: A CASE STUDY OF THE YANGTZE RIVER BASIN
}

\section{DISSERTATION}

\author{
to obtain
}

the degree of doctor at the University of Twente,

on the authority of the rector magnificus,

Prof. dr. H. Brinksma,

on account of the decision of the graduation committee,

to be publicly defended

on Wednesday 13 January, 2016 at $14.45 \mathrm{hrs}$

by

\section{Ying Huang}

Born on 05 November 1985

In Fenyi, Jiangxi, China 
This dissertation has been approved by:

Prof. dr. ir. A. Y. Hoekstra Supervisor

Prof. dr. Z. Su

Supervisor

Dr. ir. M. S. Salama

Co-Supervisor

Dr. M. S. Krol

Referee 
Struggle is not a sign of weakness, struggling is a sign of strength. That means you don't give up, you keep trying. You try, you try, until you get it.

努力奋斗并不意味着你软弱, 它反而是 一个有能力的象征。这意味着你不会放 弃, 你会一直努力尝试, 一直努力直到 你获得成功。 

Dedicated to my parents and grandma 献给我的父母亲和奶奶 
Dedicated to Weiqing

献给伟卿 


\section{Table of Contents}

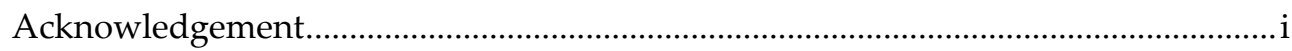

Chapter 1 General introduction ........................................................................... 1

$1.1 \quad$ Scientific background ............................................................................ 1

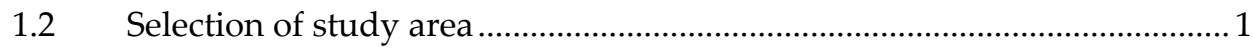

1.3 Focused hydrological component and data availability .............................. 2

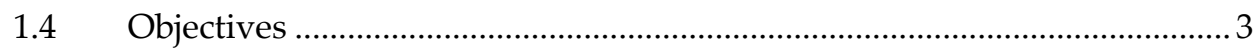

$1.5 \quad$ Dissertation Outline ................................................................................ 4

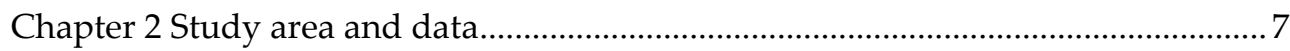

$2.1 \quad$ Study area

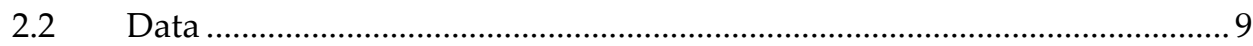

2.2.1 Global data assimilation products........................................................

2.2.2 Data for land surface modeling ……………..................................... 10

2.2.3 Remotely sensed data ..................................................................... 11

2.2.4 Field data ……………………………….................................. 13

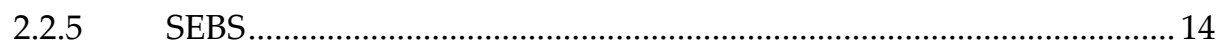

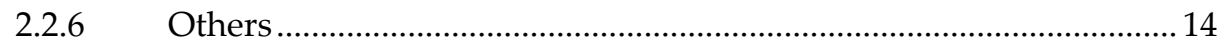

Chapter 3 Evaluation of data assimilation products for terrestrial water storage

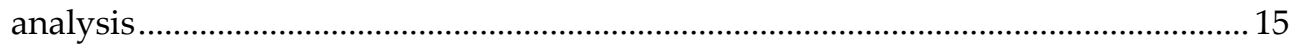

3.1 Abstract............................................................................................... 15

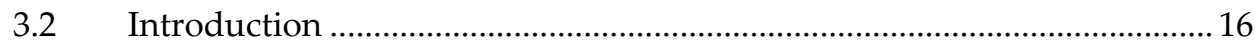

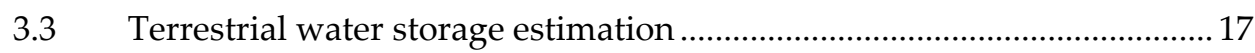

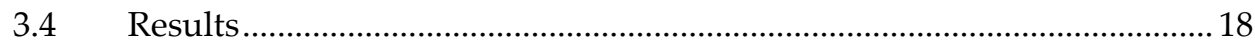

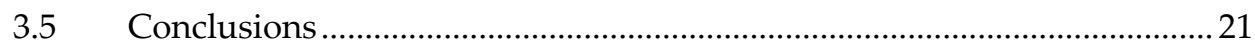


Chapter 4 Effects of roughness length parameterizations on regional scale land surface modelling of alpine grasslands in the Yangtze River basin

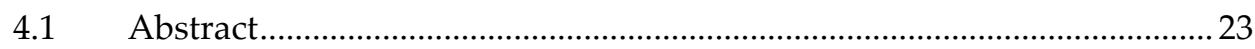

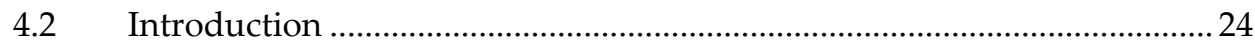

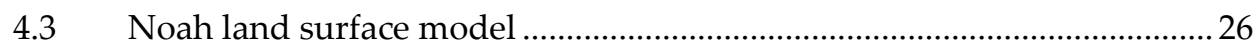

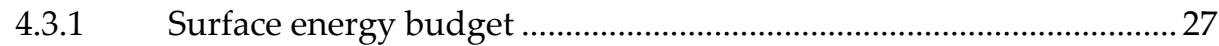

4.3.2 Runoff simulation and water budget................................................29

4.3.3 Roughness length parameterizations for the Noah LSM ...................30

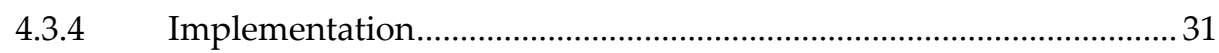

4.4 Study area and data processing .................................................................... 32

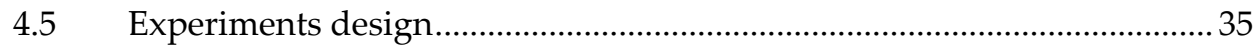

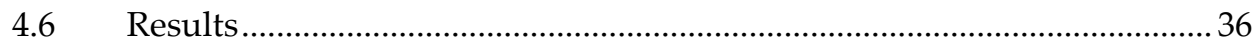

4.6.1 Impacts on $T_{s f c}$ and surface energy budget modelling.........................36

4.6.2 Impacts on water budget modelling ......................................................39

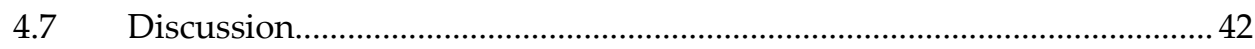

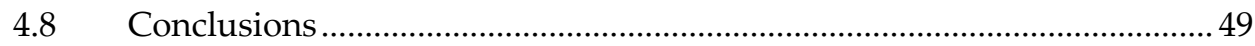

Chapter 5 Reconstruction of the Yangtze River basin water budget through integration of satellite, ground data and Noah-MP model simulations ....................51

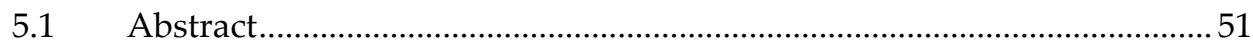

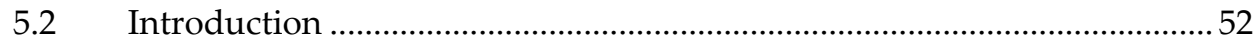

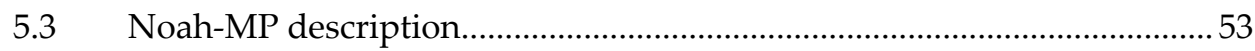

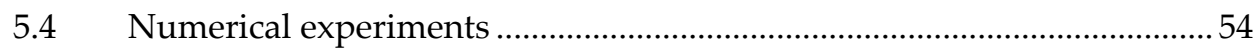

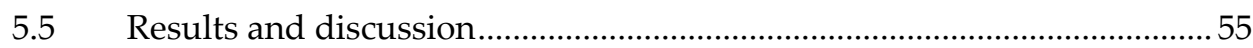

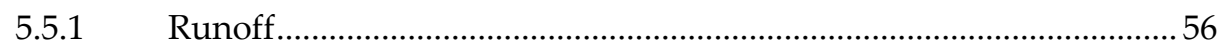

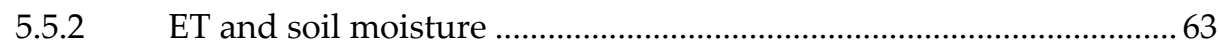

5.5.3 Comparison with GLDAS-Noah …………………….......................... 65

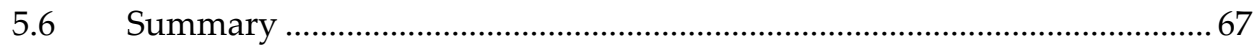


Appendix A: Options for supercooled liquid water and frozen soil scheme in

Table 5.1

Appendix B: Options for runoff and groundwater in Table 5.1

Chapter 6 Analysis of long-term terrestrial water storage variations caused by climate variability in the Yangtze River basin ............................................................. 75

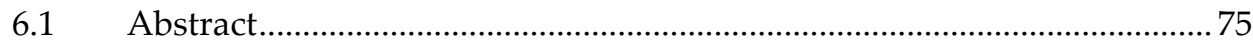

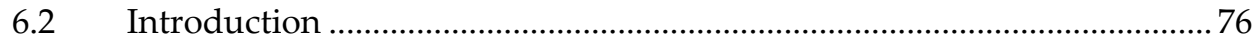

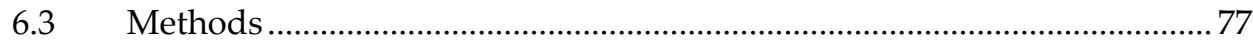

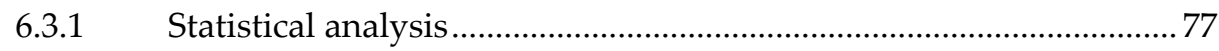

6.3.2 Standardized anomalies .................................................................... 78

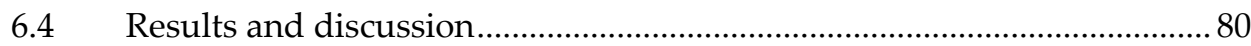

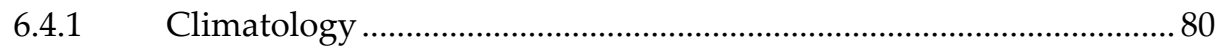

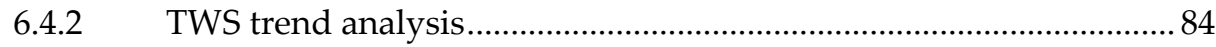

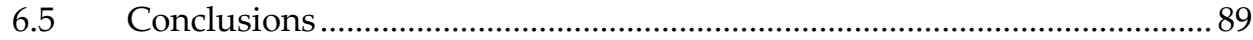

Chapter 7 Human-induced changes in terrestrial water storage of the Yangtze

River basin estimated from GRACE satellite data and land surface model

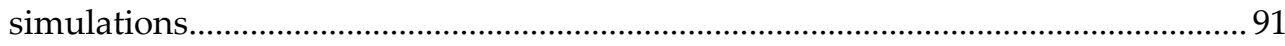

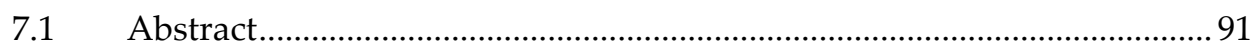

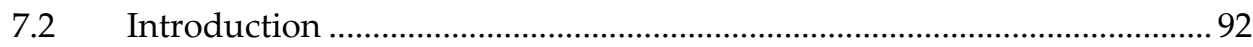

7.3 The framework for detection and attribution of spatial TWS changes.... 96

7.4 Application of the framework to the Yangtze River basin ........................ 100

7.4.1 Preliminary estimates of human-induced TWS variations ............... 100

7.4.2 ROHs and study period selection ……………................................. 102

7.4.3 ROHs classification based on ET drivers ………………………......... 104

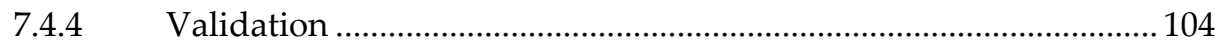

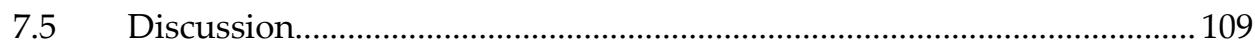

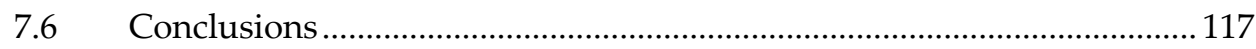

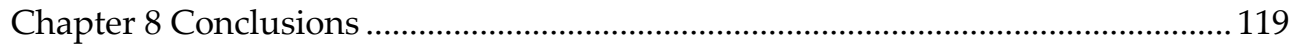


8.1 Climate-related TWS estimation …………............................................119

8.2 TWS variations caused by climate variability ............................................ 121

8.3 Human-induced changes in TWS ........................................................... 121

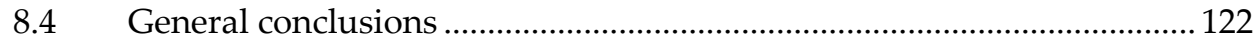

8.5 Suggestions for future research ............................................................ 122

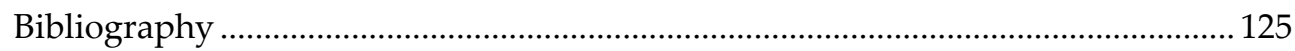

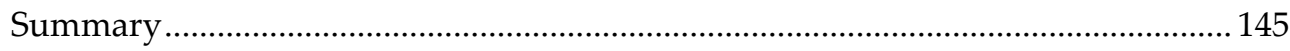

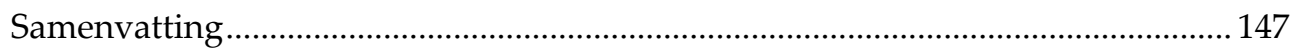

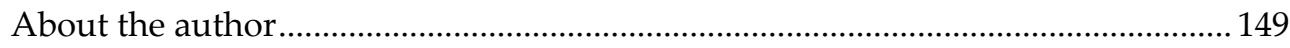




\section{Acknowledgement}

Doing a PhD is a long journey, at least for me. The long distance from my homeland and the culture difference between China and the Netherlands make this journey even more difficult. This PhD thesis cannot be finished without the help and support of many people as well as organizations. Although it is impossible for me to mention all of them here, I greatly appreciate their kindness.

My opportunity to do a PhD at the University of Twente in the Netherlands came from the recommendation of Prof. Yunxuan Zhou and the trust of Prof. Z. (Bob) Su and Prof. Arjen Y. Hoekstra. With the certification of the financial support of China Scholarship Council (CSC), Prof. Z. (Bob) Su and Prof. Arjen Y. Hoekstra gave me the invitation letter to be a PhD candidate at the University of Twente. Therefore, first of all, I would like to thank Prof. Yuanxuan Zhou, Prof. Z. (Bob) Su, Prof. Arjen Y. Hoekstra as well as CSC for offering me this great opportunity.

I came to the Netherlands and started my PhD journey in October 2010. I was very nervous since that was my first time living in a city, which is about $8000 \mathrm{~km}$ away from home. The kindness and patience of Prof. Z. (Bob) Su and Prof. Arjen Y. Hoekstra eased my mind. Their guidance helps me construct a holistic picture of my PhD research. Therefore, I sincerely appreciate their mentorship.

I am very grateful to my daily supervisors, Dr. Mhd. Suhyb Salama and Dr. Maarten S. Krol. Without the large amount of discussion with them, I cannot complete my PhD. Their continuous encouragements, enthusiastic supports and constructive suggestions ensure the success of the PhD project. On top of that, they teach me the scientific way of thinking, which can benefit my future career.

Next, I would like to thank Ms. J. de Koning, Ms. Joke Meijer, and Ms. E. L. Butt. They helped me a lot during my PhD. Their kindness makes my working life in the Netherlands much easier. 
A special thank goes to Dr. Tiejun Wang. He is generous to share his working and life experience, and construct me when I am at a loss. This is very helpful for me to tackle my working and life difficulties. Besides, I would like to thank Ms. Lichun Wang for her concern. She not only gives me suggestions for my research, but also helps for my personal life (e.g. home moving). Moreover, my thank goes to Mr. Willem Nieuwenhuis for his help on my IDL codes when I encounter IDL programming problems. I enjoy the lunch talks with them a lot.

Furthermore, I would like to thank my colleges of Water Resources department of ITC and Water Engineering \& Management Group (WEM). They have created a good atmosphere for working and generously show me their perspectives. Because of them, I have an enjoyable stay in the university. Particularly, I want to mention Dr. Yijian Zeng, Dr. Xuelong Chen, Dr. R. van der Velde, Dr. M. W. Lubczynski, Dr. Martijn Booij, W. J. Timmermans, ing. M. Ucer, Dr. C. van der Tol, La Zhuo, Xu Yuan, E. T. Hondebrink, Dr. Mireia Romaguera, Wenlong Chen, Donghai Zheng, Binbin Wang, Junping Du, Shaoning Lv, Xiaolong Yu, Qiang Wang, Tian Ye, Hongyan Zhu, H. A. Bhatti, Joep Schyns, Rick Hogeboom, Hatem Chouchane, Dr. Guowang Jin, Peiqi Yang, M. Lekula, O.A.A. Mohamed, M. W. Kimani, Dr. W. Chen, Dr. F. Zhao, Dr. M. Meng Dan and Dr. J. Zhang. I enjoyed the discussion and chat with them.

Besides, I would like to thank my friends outside the group. Particularly, I would like to mention Jingwei Zhang, Biao Xiong, Xingwu Sun, Wei Ya, Erwin Vonk, Csaba Daday, Fangyuan Yu, Zhonghua Chen, Honglin Chen, Duan Zheng, Leicheng Guo, Yin Tao, Ying Zhang, Mengmeng Li, Zheng Chen and Jing Liu.

Finally, I would like to express my appreciation to my family, my parents and Weiqing. Without their unconditional support and understanding, my $\mathrm{PhD}$ thesis would not come into being.

Ying Huang

December, 2015 


\section{CHAPTER 1}

\section{General Introduction}





\section{Chapter 1 General introduction}

\subsection{Scientific background}

River basins are substantially impacted by natural climate variability and human activities. With little human activity, hydrological systems are primarily controlled by natural climate variability. As the human population has dramatically increased and many regions involve intensive human activities, human influence can no longer be neglected and should be seriously considered as an important player in the hydrologic cycle (e.g. Savenije et al., 2014).

IPCC (2012) has documented there is evidence from observations gathered since 1950 of change in some extremes of, for instance, precipitation and discharge. There is medium confidence that some regions of the world have experienced more intense and longer droughts, whereas, in some other regions, there have been statistically significant trends in the number of heavy precipitation events. Both natural variability and human influence are important factors for those increased climate extremes which likely trigger disasters in river basins. It therefore requires a better understanding of climate and human impacts on hydrological systems.

Human impacts on the environment both directly and indirectly (Wagener et al., 2010). Indirect human influence on the water cycle refers mainly to the effect of anthropogenic changes in climate. This is, for instance, associated with greenhouse gas emissions. Direct human influence is attributed to human actions on river basins, including but not limited to hydroelectricity generation, irrigation, groundwater abstraction, and land use and cover change (LUCC). Due to the complex interaction of climatic, environmental, and human factors, the effects of human influence and climate cannot simply be added. A major scientific challenge lies in separating human effects on river basins from the climate variability. In order to achieve that, data availability and focused hydrologic component selection are prerequisite.

\subsection{Selection of study area}


In this dissertation, the Yangtze River basin is taken as the study area. This basin has experienced a trend of increasing frequency of extreme events and faces numerous forms of human alterations (IPCC, 2001; Dai et al., 2008; Yang et al., 2010), such as the Three Gorges Dam (TGD) construction and intensive irrigation. Some studies (Dai et al., 2008; Yang et al., 2010; Guo et al., 2012; Wang et al., 2013) have documented that the operation of the TGD could have a direct impact on Yangtze River flow and river-lake interaction in the middle and lower reaches of the Yangtze River basin. Moreover, the Yangtze River basin has been documented as one of areas with the highest irrigation density in the world, which may affect the distribution of water resources in the basin (Siebert et al., 2005). Therefore, the Yangtze River basin is an interesting case study area to investigate the effects of climate variability and human activities on the hydrological system.

\subsection{Focused hydrological component and data availability}

Important variables in the hydrology of catchments include precipitation, evapotranspiration (ET), runoff, and terrestrial water storage (TWS). There have been a considerable number of studies addressing the influence of climate variability and human activities on the water resources of the Yangtze River basin, however, most of them focus on river flows rather than TWS changes (e.g. Dai et al., 2008; Yang et al., 2010). As a key component of terrestrial and global hydrological cycles, TWS strongly influences water, energy, and biogeochemical fluxes, thereby playing a major role in the Earth's climate system (Famiglietti, 2004). It is not only an indicator of the Earth's climate variability, but also affects various components of the Earth's hydrological cycle (Niu and Yang, 2006).

From a historical perspective, there is limited information about the TWS distribution in time and space, as TWS is not routinely assessed like other hydrometeorological variables. Isolated datasets are available for only a few regions and rarely for periods of more than a few years. Moreover, the in situ observations are point measurements, and not always representative for larger spatial domains (Famiglietti et al., 2008; van der Velde et al., 2008). Fortunately, progress in satellite remote sensing and corresponding retrieval techniques enables large scale monitoring of land-surface bio-geophysical properties (e.g. TWS, temperature). Previous researches (e.g. Tapley et al. 2004a, b) have shown that, 
using measurements of the Earth's gravity field, spatial TWS changes can be inferred on a monthly scale. The first space mission that employs this technology is the Gravity Recovery and Climate Experiment (GRACE) launched on 17 March 2002.

Global Data assimilation products such as Interim Reanalysis Data (ERA-Interim) and Global Land Data Assimilation System (GLDAS) combine the virtues of in situ data, remotely sensed observations, and modeling. The models in these systems simulate the main components of TWS and, by fusing these components with other data sources, reduce uncertainties in the hydrological interpretations. These systems have been extensively applied in TWS and related studies, and have, for example, been utilized in analysis of regional, continental, and global TWS variations (Seneviratne et al., 2004; Chen et al., 2005; Syed et al., 2008).

The main components of TWS such as soil moisture (SM) and snow water equivalent (SWE) can also be simulated by land surface models (LSMs). This simulated TWS may have higher accuracy than publicly available global data assimilation products, because of the improved atmospheric forcing data and more realistic representations of physical processes for the study area.

Therefore, it is interesting, promising, and important to focus on spatial and temporal TWS variations and, by using remote sensing and land surface modeling techniques and compiling data from various sources, explore the impacts of climate variability and human activities on the hydrological system of the Yangtze River basin.

\subsection{Objectives}

The overall objective of this $\mathrm{PhD}$ research is to investigate the spatiotemporal effects of climate variability and human activities on the TWS of the Yangtze River basin. In order to achieve this, the specific objectives are designed and listed as follows:

1) To obtain the reliable TWS estimates by evaluating data assimilation products and/or reconstructing the water budget from LSMs for the study area. 
2) To investigate the effects of climate variability on the TWS variations based on the reliable TWS estimates over the past three decades.

3) To investigate the spatial human effects on the TWS variations, by combing the reliable TWS estimates, earth observation and field measurements.

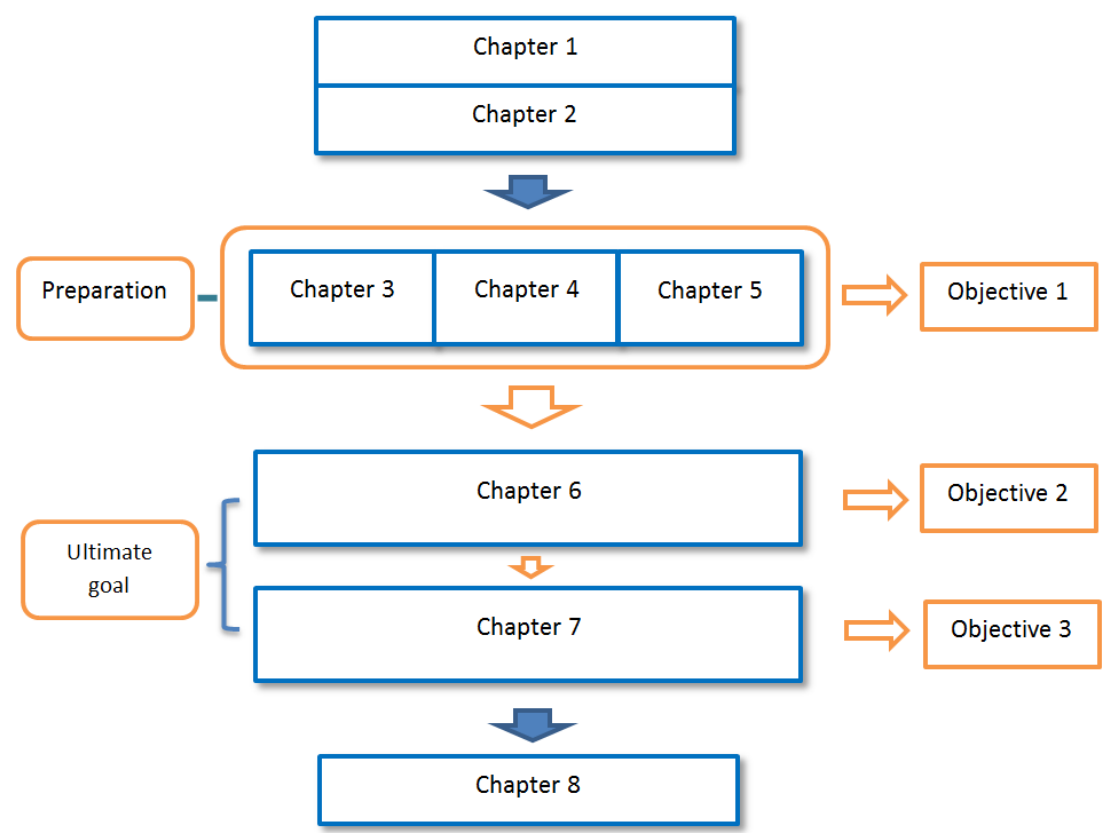

Figure 1.1. Flowchart of research and dissertation structure

\subsection{Dissertation Outline}

Figure 1.1 shows the structure of this $\mathrm{PhD}$ research and how it is related to the objectives. Chapter 2 introduces the study area and data used in this dissertation. Chapters 3, 4, and 5 are the preparation for the ultimate goal, and aim to obtain reliable TWS estimates by evaluating the data assimilation products, the Interim Reanalysis Data (ERA-Interim) and Global Land Data Assimilation System (GLDAS), and/or reconstructing the water budget from LSMs of the study area. More specifically, Chapter 3 evaluates the public data assimilation products in the 
study area for the data reliability on further investigation. Chapters 4 and 5 focus on improving the physical processes of LSMs and reconstructing the water budget of the study area, in order to obtain reliable TWS estimates. Based on the knowledge of Chapters 3-5, Chapters 6 and 7 select the most reliable TWS estimates, concentrate on the ultimate goal of this thesis and represent the main scientific contributions. Chapter 8 summarizes this $\mathrm{PhD}$ research, draws final conclusions, and gives an outlook for the future. 


\section{CHAPTER 2}

Study Area and Data 



\section{Chapter 2 Study area and data}

\section{$2.1 \quad$ Study area}

The Yangtze River, the longest river in Asia and the third longest river in the world, forms a basin of 1.8 million square kilometers $\left(\mathrm{km}^{2}\right)$, which is one-fifth of the land area of the People's Republic of China and home to one-third of the China's population. The river originates in the Qinghai-Tibetan Plateau and flows $6300 \mathrm{~km}$ eastwards to the sea. The upper Yangtze reaches, the headwaters, extend from the westernmost point, at Tuotuohe, to Yichang. The middle reaches extend from Yichang to Hukou, and the lower reaches extend from Hukou to the river mouth near Shanghai (Figure 2.1). The climate in the Yangtze River basin is governed by the monsoon, and different climatic systems control the upper and the lower Yangtze River. The amount of annual precipitation (rainfall and snowfall) within the basin tends to decrease inland. Precipitation at the headstream is less than 40 $\mathrm{cm} \mathrm{yr}^{-1}$, whereas the lower reaches receive $160 \mathrm{~cm} \mathrm{yr}^{-1}$. The wet season from April to October forms a specific weather phenomenon of the middle and lower reaches, and $85 \%$ of the annual precipitation occurs during this period.

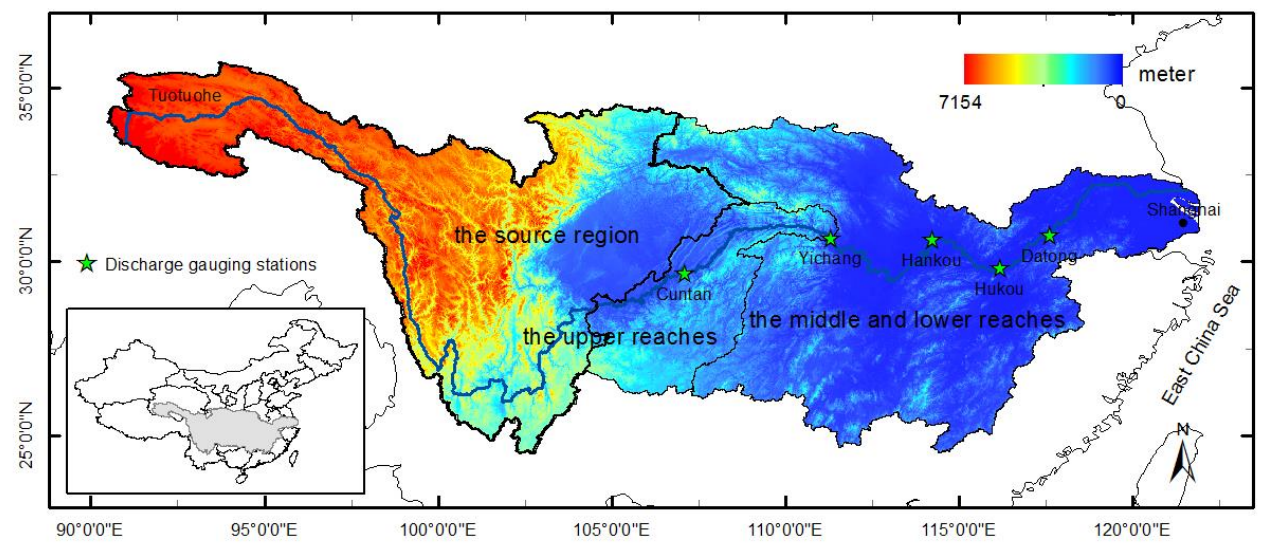

Figure 2.1.Elevation map of the study area, the Yangtze River basin. The green stars denote the locations of the main gauging stations. 
As also shown in Figure 2.1, Cuntan, Yichang, Hankou, and Datong are four main hydrological gauging stations located along the mainstream of the Yangtze, receiving discharge from catchment area of $0.86,1.01,1.49$, and $1.80 \times 10^{6} \mathrm{~km}^{2}$, respectively. Cuntan forms the entrance to TGR, which extends more than $600 \mathrm{~km}$ along the mainstream of the Yangtze River. Yichang is located $37 \mathrm{~km}$ downstream from TGD, and Hankou is located in the middle reaches of the river. Datong is the gauging station before the river flows into the sea, and it is used to represent the runoff change in the entire Yangtze River basin. The three largest natural lakes in the Yangtze River basins are Dongting Lake, Poyang Lake and Tai Lake (Figure 2.2).

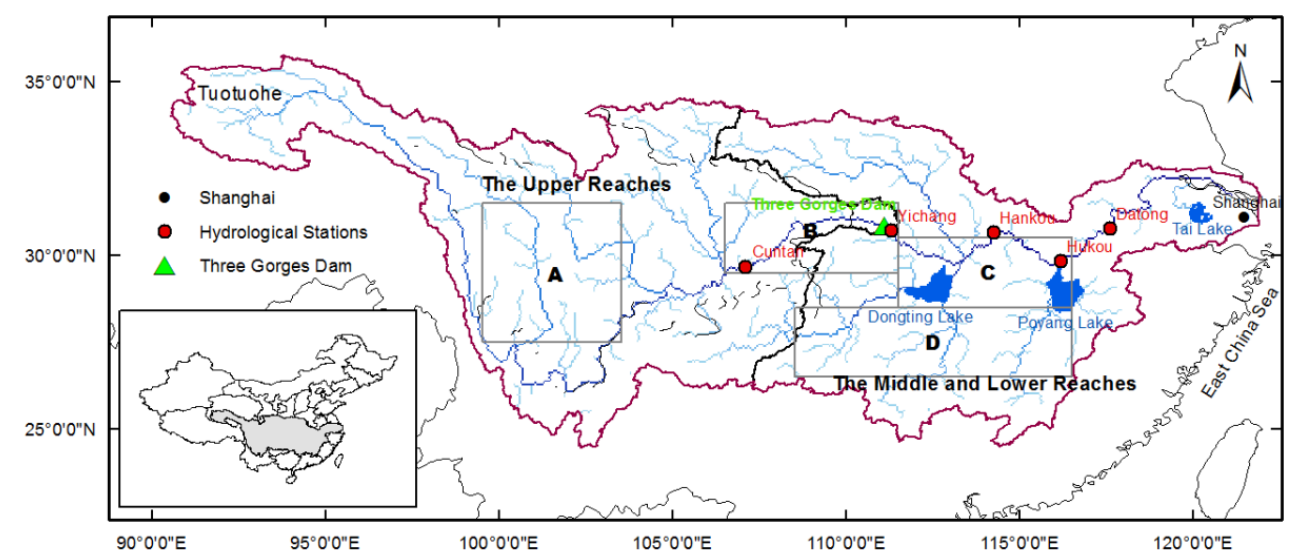

Figure 2.2.The blue polygons represent the three largest natural lakes (Dongting Lake, Poyang Lake, and Tai Lake). Rivers are delineated in blue, and the Yangtze River basin boundary in purple. The position of the Three Gorges Dam is depicted by a green triangle. Squares A, B, C and D represent the selected regions in Chapter 7 .

As the largest hydrological system in China, the Yangtze River is historically, economically and culturally important to the country. The Yangtze River is a major water resource for households, industry, and agriculture of the basin, and the prosperous Yangtze River Delta generates roughly 20\% of the gross domestic product of China. Moreover, water from the Yangtze River has enormous potential for generation of hydroelectricity, and it is transferred to the Yellow River basin in arid northern China, to alleviate water scarcity in that basin. Thus, the hydrological situation of the basin is profoundly important for the people living there, and 
sustainable water management is indispensable. However, using water resources sustainably is challenging because of the many factors involved, including climate change, the natural variability of the resource, as well as pressures due to human activities.

\subsection{Data}

\subsubsection{Global data assimilation products}

\subsubsection{ERA-Interim}

ERA-Interim reanalysis dataset, produced by European Centre for Medium-Range Weather Forecasts (ECMWF), contains physical data of atmosphere and surface analyses covering the period from 1979 to the present based on the ECMWF Integrated Forecast System (IFS) release Cy31r2 (Berrisford et al., 2011; Simmons et al., 2006). The reanalysis incorporates a forecast model with three fully coupled components for atmosphere, land surface and ocean waves, and assimilates various types of observations, including satellite and ground based measurements. It uses the Tiled ECMWF Scheme for Surface Exchanges over Land (Viterbo et al., 1995) to simulate heat and water exchanges between land and atmosphere. The TESSEL model structure includes four soil layers (0-7, 7-28, 28-100, and 100-289 cm) for each type of vegetation scheme and each type of snow scheme. As the latest global atmospheric reanalysis produced by ECMWF, it has been confirmed that the performance of this system is substantially improved in certain key aspects (the representation of the hydrological cycle, the quality of the stratospheric circulation, and the consistency in time of the reanalyzed fields) compared to ERA-40 (Dee et al., 2011).

\subsubsection{ERA-Interim/Land}

The ERA-Interim/Land dataset, produced by the European Centre for MediumRange Weather Forecasts (ECMWF), describes the evolution of the soil (moisture and temperature) and snowpack covering the period from 1979 to 2010 (Balsamo et al., 2015). It is based on the latest ECMWF land surface model, HTESSEL, driven by meteorological forcing from the ERA-Interim atmospheric reanalysis and precipitation adjustments based on the Global Precipitation Climatology Project 
(GPCP) v2.1. ERA-Interim uses the Tiled ECMWF Scheme for Surface Exchange over land (Viterbo et al., 1995; van den Hurk et al., 2000) to simulate heat and water exchanges between land and atmosphere. This system has been confirmed to perform well in certain key aspects (the representation of the hydrological cycle, the quality of the stratospheric circulation, and the consistency in time of the reanalyzed fields) (Dee et al., 2011). ERA-Interim/Land preserves closure of the water balance and includes a number of parameterizations improvements in the land surface scheme with respect to the original ERA-Interim dataset (Balsamo et al., 2015), which makes it suitable for this study. Moreover, Balsamo et al. (2015) showed the quality of ERA-Interim/Land through a comparison with groundbased and remote sensing observations. The ERA-Interim/Land reanalysis data can be freely downloaded from the website http://apps.ecmwf.int/datasets/data/interim full daily/.

\subsubsection{GLDAS-Noah}

The Global Land Data Assimilation System (GLDAS) supplies users with a model output of state-of-the-art land surface schemes created with atmospheric variables that originate from various data sources. The model has been forced by multiple datasets: bias-corrected ECMWF Reanalysis data for the time period 1979-1993, bias-corrected National Center for Atmospheric Research (NCAR) Reanalysis data for 1994-1999, NOAA/GDAS atmospheric analysis fields for 2000 and a combination of NOAA/GDAS atmospheric analysis fields, spatially and temporally disaggregated NOAA Climate Prediction Center Merged Analysis of Precipitation (CMAP) fields, and observation-based downward shortwave and longwave radiation fields, using the method of the Air Force Weather Agency's AGRicultural METeorological modeling system (AGRMET), for the period 2001 to the present (Rui, 2011).

\subsubsection{Data for land surface modeling}

\subsubsection{Atmospheric forcing}

The Institute of Tibetan Plateau Research, Chinese Academy of Sciences (hereafter ITPCAS) provides an atmospheric forcing data set for China (He, 2010). The ITPCAS forcing data merged the observations collected at 740 operational stations 
of the China Meteorological Administration (CMA) to the corresponding Princeton meteorological forcing data (Sheffield et al., 2006) producing near-surface air temperature, pressure, wind speed, and specific humidity. The precipitation field has been produced by combining three precipitation data sets, including precipitation observations from 740 operational stations, the Tropical Rainfall Measuring Mission (TRMM) 3B42 precipitation products (Huffman et al., 2007), and GLDAS precipitation data. The GLDAS precipitation data were only used to replace TRMM 3B42 data that are not available beyond $40^{\circ} \mathrm{N}$. The Global Energy and Water Cycle Experiment - Surface Radiation Budget (GEWEX-SRB) shortwave radiation data and Princeton forcing data were been combined and corrected by radiation estimates from CMA station data using a hybrid radiation model (Yang et al., 2006), to produce downward shortwave radiation. The downward longwave radiation is calculated by the model of Crawford and Duchon (1999) based on the produced near-surface air temperature, pressure, specific humidity, and downward shortwave radiation. The temporal and spatial resolutions of the ITPCAS forcing data are 3 hourly and $0.1^{\circ}$, respectively. This dataset can be obtained at http://westdc.westgis.ac.cn/data/7a35329c-c53f-4267-aa07-e0037d913a21.

\subsubsection{Vegetation and soil data}

The United States Geological Survey (USGS) 30 arc-second global 24-category vegetation type (land-use), the hybrid State Soil Geographic Database (STATSGO) Food and Agriculture Organization (FAO) soil texture data sets, and a monthly green vegetation fraction (GVF) database based on the 5-yr (1985-90) Advanced Very High Resolution Radiometer (AVHRR) the Normalized Difference Vegetation Index (NDVI) data are used as model input. The annual mean deep soil temperate data are used in the model as bottom boundary layer conditions for soil models. All the static data we used are provided by National Center for Atmospheric Research (NCAR), and can be downloaded at http://www2.mmm.ucar.edu/wrf/users/download/get sources wps geog.html.

\subsubsection{Remotely sensed data}

\subsubsection{GRACE}


The GRACE Tellus land products, providing monthly terrestrial water storage (TWS) variations with spatial sampling of $1^{\circ}$, have been processed by the Center for Space Research (CSR, University of Texas, USA), Jet Propulsion Laboratory (JPL, NASA, USA) and German Research Centre for Geosciences (GFZ, Potsdam, Germany), and are freely available at the website ftp://podaacftp.jpl.nasa.gov/allData/tellus/L3/land mass/RL05/netcdf/. The data are based on the RL05 spherical harmonics from CSR, JPL and GFZ, and have additional post processing steps, summarized online at $\underline{\mathrm{ftp}: / / \text { podaac- }}$ ftp.jpl.nasa.gov/allData/tellus/L3/land mass/RL05/netcdf/. We used 96 months, from January 2003 to December 2010, of the GRACE Tellus land data computed by CSR. Due to the post-processing of GRACE observations, surface mass variations at small spatial scales tend to be attenuated. Therefore, it is necessary to multiply those GRACE Tellus land data by the scaling grid provided by JPL. The scaling grid is a set of scaling coefficients, one for each 1 degree bin of the land grids, and is intended to restore much of the signal removed by the post processing steps, such as destriping, filtering, and truncation described in Landerer and Swenson (2012).

It should be noted that, in Chapter 5, GRACE data are not used to evaluate the simulated TWS, but the simulated evapotranspiration (ET) through a water balanced approach (Rodell et al., 2004). The GRACE based ET has been proved to be valuable for assessing modeled ET (Rodell et al., 2004), and can be used to examine from the Surface Energy Balance System (SEBS) modelled ET output.

\subsubsection{GPCC}

Precipitation data from the Global Precipitation Climatology Centre (GPCC) were used to further support the results. GPCC offers a gauged-based, gridded, monthly precipitation dataset for the global surface for the period 1901-2010. It is based on about 67200 stations with at least 10 years of data, and recommended for use in global and regional water balance studies, the calibration/validation of remote sensing based rainfall estimates, and the verification of numerical models (Schneider et al., 2011). In this study, we used the GPCC Full Data Reanalysis Version 6 with a spatial resolution of $1.0^{\circ}$. 


\subsubsection{Water level of lakes}

The monthly water level data for the three largest natural lakes (Dongting Lake, Poyang Lake and Tai Lake) (Figure 2.2) in the study area were obtained from the web database (HYDROWEB: http://www.legos.obsmip.fr/en/soa/hydrologiehydroweb/), developed by LEGOS (Laboratoire d'Etude en Ge'ophysique et Oce'anographie Spatiale). This database is based on multi-satellite altimetry measurements and freely available for the study period (Crétaux et al., 2011). As the time series is not complete for the study period, we used linear interpolation to fill the missing months.

\subsubsection{MODIS land surface temperature}

Moderate Resolution Imaging Spectroradiometer (MODIS) land surface temperature $\left(T_{s f c}\right)$ products were used as the ground 'reference' to evaluate the $T_{s f c}$ modelling. The MODIS/Terra $T_{\text {sfc }}$ and Emissivity Daily L3 Global 0.05Deg CMG (MOD11C1) products provide $T_{\text {sfc }}$ two times per day, daytime around 10:30 A.M and nighttime around 10:30 P.M (local solar time). In Chapter 4, we selected 312 daytime cloud free MODIS images from the MOD11C1 $T_{\text {sf }}$ product archive, covering the period 2005-2010. Our choice of daytime $T_{s f c}$ product is supported by the fact that parameterization of roughness lengths plays a more important role in the daytime $T_{\text {sfc }}$ simulation than in the nighttime one (Chen et al., 2011). MODIS data are freely available on this website: https://lpdaac.usgs.gov/products/modis products table.

\subsubsection{MODIS NDVI}

The Moderate Resolution Imaging Spectroradiometer (MODIS) derived the NDVI product is designed to provide consistent spatial and temporal comparisons of vegetation conditions. The monthly MODIS NDVI during the study period 20032010, with $0.05^{\circ}$ of spatial resolution, was used as a surrogate of vegetation coverage in Chapter 7.

\subsubsection{Field data}

\subsubsection{Discharge}


The observed discharge data of the main hydrological gauging stations (Cuntan, Yichang, and Datong) in the Yangtze River basin were provided by Bureau of Hydrology, Changjiang (also called Yangtze) Water Resources Commission.

\subsubsection{Water supply and use}

The yearly water supply data from surface and groundwater, and the consumption data for living, industry, agriculture, and biology in this basin are obtained from the Ministry of Water Resources of China.

\subsubsection{SEBS}

We used monthly ET estimated from the Surface Energy Balance System (SEBS) to evaluate the Noah and Noah-MP simulations in Chapter 5. This simulation is forced by ITPCAS meteorological data, and has been validated by 11 China flux stations, including bare soil, alpine meadow, forest, cropland, orchard, grassland, and wetland land covers (Chen et al., 2014). It is of interest to note that GRACEbased ET and SEBS-estimated ET are fully independent of each other, as the former is estimated through the water balance, whereas the latter is based on the energy balance.

\subsubsection{Others}

The Global Map of Irrigation Areas (GMIA) version 5, provided by the global water information system (AQUASTAT) of the Food and Agriculture Organization (FAO), was used to calculate the irrigated area and expressed in hectares per cell (Siebert et al., 2013).

Drainage fraction $f_{d}$ was derived from global-scale information on drainage in rain fed and irrigated agriculture as compiled by Feick et al. (2005).

The in situ measurements of TGR water volume changes was obtained from the China Three Gorges Corporation (http://www.ctg.com.cn), as used by Wang et al. (2011). 


\section{CHAPTER 3}

\section{Evaluation of Data Assimilation Products for Terrestrial Water Storage Analysis}





\section{Chapter 3 Evaluation of data assimilation products for terrestrial water storage analysis}

This chapter is based on:

Huang, Y., M. S. Salama, M. S. Krol, R. van der Velde, A. Y. Hoekstra, Y. Zhou, and $\mathrm{Z}$. Su, 2013: Analysis of long-term terrestrial water storage variations in the Yangtze River basin. Hydrol. Earth Syst. Sci., 17, 1985-2000.

\subsection{Abstract}

This chapter evaluates the reliability of the data assimilation products, Interim Reanalysis Data (ERA-Interim) and Global Land Data Assimilation System (GLDAS), for studying the natural terrestrial water storage (TWS) of the Yangtze River basin. The accuracy of these datasets is validated using 26 years (1979-2004) of runoff data from the Yichang gauging station and compared with 32 years of independent precipitation data obtained from the Global Precipitation Climatology Centre Full Data Reanalysis Version 6 (GPCC) and NOAA's PRECipitation Reconstruction over Land (PREC/L). In addition, in order to assess the impact of the lack of some components such as surface water and groundwater on the matchup, we compared TWS derived from ERA-Interim to those derived from GRACE observations for a 7-year period at the basin scale. 


\subsection{Introduction}

Terrestrial water storage (TWS) is determined by all physical phases of water stored above and below the surface of the Earth, including soil moisture, snow and ice, canopy water storage, groundwater, etc. From a historical perspective, there is limited information about the TWS distribution in time and space, as TWS is not routinely assessed like other hydrometeorological variables. Isolated datasets are available for only a few regions and rarely for periods of more than a few years. Moreover, the in situ observations are point measurements, and not always representative for larger spatial domains (Famiglietti et al., 2008; van der Velde et al., 2008). Fortunately, progress in satellite remote sensing and corresponding retrieval techniques enables large scale monitoring of land-surface bio-geophysical properties (e.g. TWS, temperature). This may potentially improve our understanding of the spatially heterogeneous hydrometeorological processes.

Advances in microwave remote sensing have demonstrated their use in providing large-scale soil moisture information, resulting in satellite missions specifically dedicated to soil moisture (Entekhabi et al., 2010). Microwave observations can, however, only provide information on the top few centimeters of the soil. In addition, Tapley et al. (2004a, b) and others have shown that, using measurements of the Earth's gravity field, terrestrial water storage change (TWSC) may be inferred on a monthly scale. The first space mission that employs this technology is the Gravity Recovery and Climate Experiment (GRACE) launched on 17 March 2002.

Data assimilation products such as Interim Reanalysis Data (ERA-Interim) and Global Land Data Assimilation System (GLDAS) combine the virtues of in situ data, remotely sensed observations, and modeling. The models in these systems simulate the main components of TWS and, by fusing these components with other data sources, reduce uncertainties in the hydrological interpretations. These systems have been extensively applied in TWS and related studies, and have, for example, been utilized in regional, continental, and global TWS variation analysis (Chen et al., 2005; Seneviratne et al., 2004; Syed et al., 2008). As well, these systems 
offer long-term records of data, making them potential for long-term analysis, while remotely sensed data and in situ observations are most likely time limited.

High reliabilities of these data assimilation products are essential for TWS and related studies. In this study, the regional accuracies and reliabilities of the ERAInterim and GLDAS-Noah datasets therefore are assessed in the study area, the Yangtze River basin. The assessed results will be compared to the improved land surface modeling results in Chapters 4 and 5, in order to examine the natural spatial and temporal variation in TWS in Chapters 6 and 7.

\subsection{Terrestrial water storage estimation}

TWS is generally defined as all phases of water stored above and below the surface of the Earth: soil moisture, canopy water storage, snow water equivalent and ground water, surface water storage, etc. Our analysis of storage is, however, limited to the total soil moisture column (TSM) and snow water equivalent (SWE) and does not give a complete description of the lateral and vertical distribution of water storage unless surface and groundwater components are added to the land model used here. We also neglect canopy water storage (CWS), although this is included in the GLDAS-Noah simulation. The reason is that CWS in the Yangtze River basin is negligible in comparison with soil moisture (Zhong et al., 2010). Therefore, TWS is expressed as equation (3.1), where $N$ is an index representing the month of the year.

$$
T W S_{N}=T S M_{N}+S W E_{N}
$$

The monthly change in terrestrial water storage $\left(T W S C_{N}\right)$ can be calculated at each pixel as follows:

$$
T W S C_{N}=\left\{T S M_{N}+S W E_{N}\right\}-\left\{T S M_{N-1}+S W E_{N-1}\right\},
$$

This method elicits promising results and also compares well with the Gravity Recovery and Climate Experiment (GRACE) estimation and the monthly basinscale terrestrial water balance approach from flux variables (Chen et al., 2009; 
Rodell et al., 2004; Syed et al., 2008). The ERA-Interim soil profile includes four layers of 7, 21, 72, and $189 \mathrm{~cm}$ depth (forming a total of $289 \mathrm{~cm}$ ), while the Noah soil profile includes four layers of $10,30,60$, and $100 \mathrm{~cm}$ (200 cm in total). In order to be able to compare the TWS information obtained from both these datasets, we only considered the first $200 \mathrm{~cm}$ of soil in both cases.

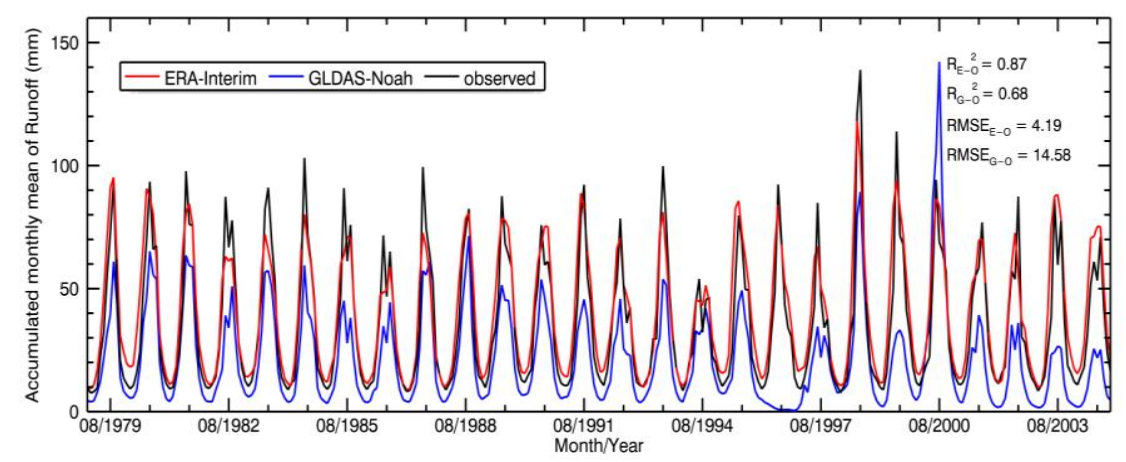

Figure 3.1.Spatially averaged time series of ERA-Interim estimated (red curve), GLDAS-Noah estimated (blue curve) and observed (black curve) runoff of the upper Yangtze reaches between January 1979 and December 2004.

\section{$3.4 \quad$ Results}

The regional accuracies and reliabilities of the ERA-Interim and GLDAS-Noah datasets are assessed by comparing their spatially averaged time series of runoff for the upper Yangtze River, generated by the observed discharge at the Yichang gauging station for the period 1979 to 2004 . This procedure is based on the method of Balsamo et al. (2009) and implemented as follows.

a. ERA-Interim/GLDAS-Noah. Firstly, we computed the accumulated monthly runoff from ERA-Interim/GLDAS-Noah data at each pixel during the period 1979 to 2004 . Secondly, we calculated the spatial-mean of the accumulated monthly runoff $(\mathrm{mm})$ of all pixels located in the upper reaches of the Yangtze River basin.

b. Discharge at the Yichang gauging station. Firstly, we computed the accumulated monthly discharge $\left(\mathrm{m}^{3}\right)$ from the daily discharge data $\left(\mathrm{m}^{3} \mathrm{~s}^{-1}\right)$ of the Yichang station. Secondly, we divided this figure by the area of the 
upper reaches. The second stop is supported by the fact that the Yichang station forms the exit points of the upper reaches of the Yangtze River basin.

Figure 3.1 shows that the ERA-Interim modeled runoff fits the observed values better than the GLDAS-Noah modeled runoff does, for the period between 1979 and 2004. The coefficient of determination (R-squared) and the root mean square error (RMSE) between the modeled and observed values for ERA-Interim (RE-O², RMSEE-O) are 0.87 and $4.19 \mathrm{~mm}$, respectively, while for GLDAS-Noah (RG-O ${ }^{2}$, RMSEG-o), they are 0.68 and $14.58 \mathrm{~mm}$, respectively. Note that the runoff is consistently underestimated by GLDAS-Noah, which is also confirmed by Zaitchik et al. (2010). GLDAS-Noah outputs show errors in 1996 and 1997. Apparently, ERA-Interim datasets show higher accuracy and reliability for the Yangtze River basin.

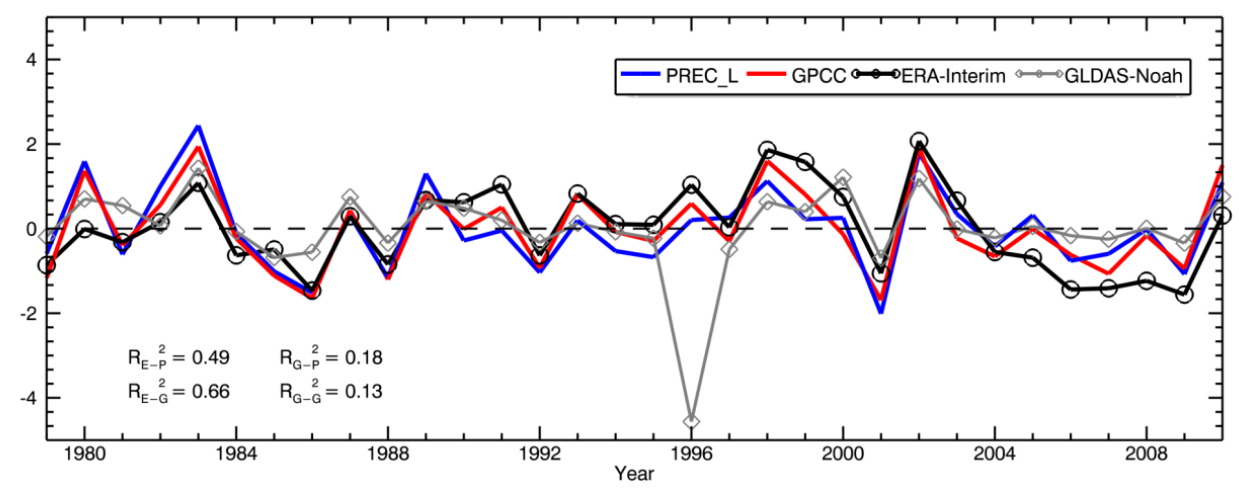

Figure 3.2.Spatially averaged time series of standardized anomalies of the annual precipitation in the middle and lower Yangtze reaches, based on PREC/L (blue curve), GPCC (red curve), ERA-Interim (black circle curve) and GLDAS-Noah (gray diamond curve) data from 1979 to 2010.

To explore the quality of these datasets further and as precipitation arguably forms the most critical input into an accurate TWS, precipitation estimates of ERAInterim and GLDAS-Noah are compared with products from the GPCC and PREC/L, which are derived more directly from observations. The spatially averaged time series of standardized annual anomalies have been computed and compared for these four datasets. The result (see Figure 3.2) shows a notable error in 1996 concerning GLDAS-Noah. GPCC and PREC/L fit very well (their R-squared 
value is 0.86). Generally speaking, ERA-Interim precipitation fits PREC/L and GPCC better than GLDAS-Noah does. The R-squared between ERA-Interim and PREC/L (RE-P $\left.{ }^{2}\right)$ and between ERA-Interim and GPCC (RE-G $\left.{ }^{2}\right)$ are 0.49 and 0.66, respectively, while the R-squared between GLDAS-Noah and PREC/L (RG-P2) and between GLDAS-Noah and GPCC ( $\left.\mathrm{RG}_{\mathrm{G}}{ }^{2}\right)$ are 0.18 and 0.13 , respectively. ERAInterim generally shows good agreement with GPCC and PREC/L.

In situ measurements of soil moisture are invaluable for the calibration and validation of a land surface model and satellite-based soil moisture retrieval. Unfortunately, there is a very low sampling rate with only 1 sample being available in the Yangtze River basin from the International Soil Moisture Network (ISMN) (Dorigo et al., 2011). However, the error structures of the ERA-Interim and GLDAS-Noah soil moisture products have been estimated using the triple collocation technique by Dorigo et al. (2010) and Scipal et al. (2008). ERA-Interim reanalyzed soil moisture is characterized by a relatively low mean global error of $0.018 \mathrm{~m}^{3} \mathrm{~m}^{-3}$ (Dorigo et al., 2010), which is fairly consistent with the average error (a mean global error of $0.020 \mathrm{~m}^{3} \mathrm{~m}^{-3}$ ) obtained by Scipal et al. (2008) by applying the triple collocation model to three satellite-based and model-based soil moisture products. It is found that the errors of soil moisture estimates in the Yangtze River basin are at an intermediate level. This can also be confirmed by the high correlation with ASCAT retrievals for the years 2007 and 2008 (Dorigo et al., 2010) and ERS-2 retrievals for the years 1998, 1999, and 2000 (Scipal et al., 2008). In addition, Liu et al. (2011) has shown that there is a high correlation coefficient (R) between GLDAS-Noah and ASCAT retrievals for the Yangtze River basin in 2007. It has been firmly proven that active microwave satellite-based (e.g. ASCAT) retrievals result in smaller errors in moderately to densely vegetated areas (e.g. the Yangtze River basin) than passive microwave products do (Liu et al., 2011). Therefore, the high correlation between ERA-Interim, or GLDAS-Noah, and active microwave satellite-based soil moisture retrievals provides some confidence in the EAR-Interim and GLDAS-Noah soil moisture qualities in the Yangtze River basin.

Other components such as surface water and groundwater form a large proportion of the TWS. To assess their impact on the matchup, we compared TWS products derived from ERA-Interim to those derived from GRACE observations 
(reprocessed Release-05, GRACE RL05) for a 7-year period (2004-2010). Figure 3.3 shows that the magnitude of the spatially averaged TWS anomalies from these two datasets (ERA-Interim and GRACE RL05) is similar and exhibits the same variation, with a coefficient of determination as high as 0.79. This means that the ERAInterim product on TWS over a soil depth of $2 \mathrm{~m}$ is representative for the GRACE observations that are affected by water storage fluctuations in the entire air-land column, including surface water and groundwater.

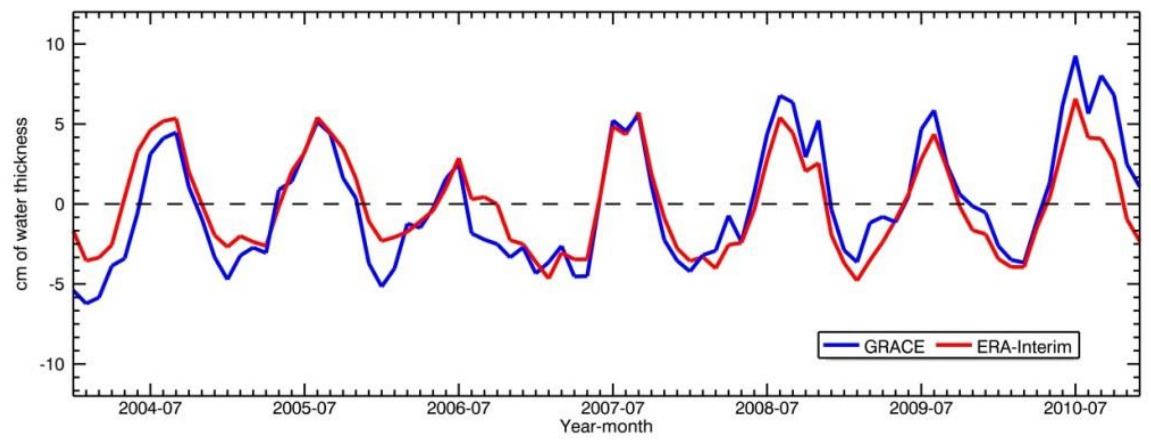

Figure 3.3.TWS anomalies [cm] averaged for a seven-year period (2004-2010) and obtained from ERAInterim (red line) and GRACE RL05 (blue line) datasets for the Yangtze River basin.

\subsection{Conclusions}

In terms of runoff simulation, the ERA-Interim data show higher accuracy and reliability than the GLDAS-Noah data for the upper reaches of the Yangtze River basin. Also, the precipitation of ERA-Interim generally shows good agreement with GPCC and PREC/L. In addition, the TWS estimated from ERA-Interim has good matchup with that from GRACE data at a basin scale. The foregoing indicates that ERA-Interim data perform better than GLDAS-Noah in the Yangtze River basin, and ERA-Interim is therefore more suitable to investigate the natural TWS variations in the study area. 


\title{
CHAPTER 4
}

\author{
Effects of Roughness Length \\ Parameterizations on Regional Scale \\ Land Surface Modelling of Alpine \\ Grasslands in the Yangtze River Basin
}





\section{Chapter 4 Effects of roughness length parameterizations on regional scale land surface modelling of alpine grasslands in the Yangtze River basin}

\section{This chapter is based on:}

Huang, Y., M. S. Salama, Z. Su, R. van der Velde, D. Zheng, M. S. Krol, A. Y. Hoekstra, and Y. Zhou, 'Effects of Roughness Length Parameterizations on Regional Scale Land Surface Modelling of Alpine Grasslands in the Yangtze River Basin.' [Accepted subject to minor revisions at Journal of Hydrometeorology (JHM)]

\subsection{Abstract}

Current land surface models (LSMs) tend to largely underestimate the daytime land surface temperature $\left(T_{s f c}\right)$ for high-altitude regions. This is partly due to underestimation of heat transfer resistance, which may be resolved through adequate parametrization of roughness lengths for momentum $(z 0 m)$ and heat $(z \circ)$ transfer. In this chapter, we address the regional-scale effects of the roughness length parameterizations for alpine grasslands, and assess the performance of the Noah LSM using the updated roughness lengths compared to the original ones. The simulations were verified with various satellite products and validated with ground-based observations. More specifically, we designed four experimental setups using two roughness length schemes with two different parameterizations of $z$ om (original and updated). These experiments were conducted in the source region of the Yangtze River during the period 2005-2010 using the Noah LSM. The results show that the updated parameterizations of roughness lengths reduce the mean biases of the simulated daytime $T_{s f c}$ in spring, autumn and winter by up to $2.7 \mathrm{~K}$, whereas larger warm biases are produced in summer. Moreover, model efficiency coefficients (Nash-Sutcliffe) of the monthly runoff results are improved by up to $26.3 \%$, when using the updated roughness parameterizations. In addition, the spatial effects of the roughness length parametrizations on the $T_{s f c}$ simulations 
are discussed. This study stresses the importance of proper parameterizations of $z z_{m}$ and $z o h$ for LSMs, and highlights the need for regional adaptation of the $z o m$ and $z$ oh values.

\subsection{Introduction}

The Tibetan Plateau is geographically known as roof of the world or third pole of the earth. It not only plays an important role in the formation of the Asian monsoon (Yanai et al., 1992; Yanai and $\mathrm{Wu}, 2006$ ), but also serves as the headwaters of several large rivers in Southeast Asia, such as the Indus, Mekong, Brahmaputra, Yellow and Yangtze Rivers. This area has experienced significant environmental changes, such as increased warming (e.g. Chen et al., 2014), enhanced frequency of drought (e.g. $\mathrm{Ma}$ and $\mathrm{Fu}, 2006$ ), intensified land degradation and desertification (e.g. Fu and Wen, 2002). In addition, Immerzeel et al. (2010) have shown that the hydrologic cycle has changed in recent years, influencing runoff of rivers originating from the region. Reliable hydrometeorological simulations are required assets for understanding land-atmosphere interactions in the Tibetan Plateau and their response to climate change and human activities.

The Tibetan Plateau is an arid and semiarid region mainly characterized by bare soil and grassland. Due to strong solar radiation, low air density and the influence of the Asian monsoon, the Tibetan Plateau has very distinct and complex diurnal and seasonal variations of the surface energy and water budget (Yang et al., 2009). Because of this, current land surface models (LSMs), e.g. Common Land Model (CLM), Simple Biosphere Model, version 2 (SiB2), and Noah LSM, tend to significantly underestimate the daytime land surface temperature $\left(T_{s f c}\right)$ and overestimate sensible heat flux $(H)$ in the Tibetan Plateau, particularly in dry conditions (Yang et al., 2007, 2009; Chen et al., 2011).

In these LSMs, the bulk formulations based on the Monin-Obukhov similarity theory (MOST) have usually been employed to simulate the surface heat fluxes between the land surface and atmosphere (Garratt, 1994; Brutsaert, 1998; Su et al., 2001). Su et al. (2001) have documented that, in order to accurately reproduce $H$ through MOST, the roughness lengths for momentum $\left(z_{0 m}\right)$ and heat $(z \circ h)$ transfer 
must be determined properly. Both parameters cannot be directly measured, but can be ideally determined using the bulk transfer equations from profile measurements of wind and temperature (Sun, 1999; Ma et al., 2002; Yang et al., 2003) and/or from single-level sonic anemometer measurement (Sun, 1999; Martano, 2000; Ma et al., 2008). The importance of $z 0 m$ and $z$ oh to LSMs has been reported by many authors. For instance, LeMone et al. (2008) has pointed out that a proper representation of $z$ oh is helpful to reproduce the observed $T_{\text {sfc }}$ and $H$. Yang et al. (2009) has further confirmed that the underestimation of heat transfer resistances accounts for the daytime $T_{s f c}$ underestimation in current LSMs for the Tibetan plateau. Based on these results, we argue that robust parameterizations of $z o m$ and $z o h$ are imperative for reliable surface heat flux estimates and $T_{s f c}$ calculation in the Tibetan plateau.

There are a number of theoretical and experimental studies on the parameterizations of $z_{0 m}$ and $z o h$ for LSMs. In general, $z 0 m$ is estimated according to surface geometric characteristics, whereas zoh is calculated based on $z$ om through the parameterizations of $k B^{-1}\left(k B^{-1}=\ln (z o m / z o h)\right)$. Brutsaert (1982, hereafter B82) combined the roughness Reynolds number $\mathrm{Re}^{*}$ and vegetation characteristics (e.g. leaf area index, canopy height) to parameterize $k B^{-1}$. Zilitinkevich (1995, hereafter Z95) proposed an empirical coefficient, known as the Zilitinkevich empirical coefficient $\left(C_{z i l}\right)$, to relate the roughness Reynolds number $\operatorname{Re}^{*}$ to $k B^{-1}$, and since Chen et al. (1997), Z95 has been widely used in LSMs. Chen and Zhang (2009, hereafter C09) found that the $z_{0 m}$ and $z$ oh of $Z 95$ tend to be overestimated for short vegetation and underestimated for tall vegetation, and hence, on the basis of Z95, parametrized $z$ om and $z o h$ as functions of canopy height. Similarly, Zheng et al. (2012, hereafter Z12) proposed to utilize green vegetation fraction (GVF) for the modification of Z95. In addition, Yang et al. (2008, hereafter Y08) assessed several schemes, including B82 and Z95, and showed that $z$ om and $z$ oh can be more realistically parameterized by taking into account friction velocity $\left(u_{*}\right)$ and friction temperature $\left(\theta_{*}\right)$ in arid and semiarid regions of China.

For the Tibetan Plateau, on the other hand, the studies on roughness length parameterizations were still very limited until 1998, when intensive field experiments and comprehensive observational networks started to develop (Koike, 2004; Ma et al., 2008; Xu et al., 2008). Since then, a number of progresses have been 
made in the parameterizations of roughness lengths for the Tibetan Plateau. For instance, Chen et al. (2010) showed that Y08 can perform better in the Tibetan Plateau based on extensive evaluation of different roughness length schemes in LSMs. This study is very valuable; however it is limited to 2-month pre-monsoon episodes, and did not consider revising roughness length schemes other than Y08 to operate on the Tibetan Plateau. This kind of revision was performed by Zheng et al. (2014), in which they used field measurements to revise the values of zom for Z95, C09 and Z12 for a Tibetan site in different seasons. Zheng et al. (2014) showed that revising the values for $z o m$ and $z$ oh dramatically improves the performance of the Noah LSM on the simulations of $T_{s f c}$ and surface heat fluxes at a point scale, and suggested using the $\mathrm{C} 09$ with the newly revised zom for actual application, due to its consistent performance in different seasons.

The impacts of roughness length parameterizations on $T_{s f c}$ and surface heat fluxes, as well as water fluxes simulations at a regional scale are yet to be investigated for the Tibetan Plateau. In this study, we extend on the previous study of Zheng et al. (2014) by assessing parameterizations of roughness lengths for $T_{s f c}$ and heat fluxes estimation at a regional scale for the Tibetan Plateau. Furthermore, we explore their effects on the simulations of water fluxes and states. We selected two roughness length parameterization schemes, Z95 and C09, with the original and the Zheng et al. (2014) derived roughness lengths for evaluation. Our selection was based on the common usage of Z95 in LSMs, whereas C09 has better performance than Y08 and Z12 (Zheng et al., 2014). Moreover, the Jinsha, Mintuo and Jialing sub basins (the source region) of the Yangtze River is taken as the study area (Figure 4.1). This is mainly due to the fact that the source region of the Yangtze River has diverse hydro-meteorological conditions, and is relatively less influenced by human activities than the neighboring catchments such as the source region of the Yellow River basin.

\subsection{Noah land surface model}

The Noah LSM has been widely used for surface heat flux and hydrology simulations, and forms the land component of mesoscale and global weather forecasting models for investigating complex interactions between land and atmosphere (Dirmeyer et al., 2006; Zhang et al., 2011). It uses a Penman-based 
approximation for latent heat flux ( $L E)$ to solve surface energy balance (Mahrt and Ek, 1984), a four-layer soil model with thermal conduction equations for simulating the soil heat transport, and the diffusivity form of Richards's equation for soil water movement (Mahrt and Pan, 1984). A simple water balance model (SWB) is used by the Noah LSM to calculate the surface runoff (Schaake et al., 1996).

\subsubsection{Surface energy budget}

In general, the surface energy balance equation can be written as:

$$
\begin{gathered}
R_{n e t}=(1-\alpha) S^{\downarrow}+\varepsilon\left(L^{\downarrow}-\sigma T_{s f c}{ }^{4}\right), \\
R_{n e t}=H+L E+G_{0}, \quad \text { (4.2) }
\end{gathered}
$$

In equation (4.1), $R_{\text {net }}$ is the net radiation $\left(\mathrm{W} \mathrm{m}^{-2}\right), \mathrm{S}^{\downarrow}$ and $\mathrm{L}^{\downarrow}$ are the downward shortwave and longwave radiation $\left(\mathrm{W} \mathrm{m}^{-2}\right)$, respectively. $T_{\text {sfc }}$ is the land surface temperature (K), $\sigma$ is the Stefan-Boltzmann constant $\left(=5.6710^{-8} \mathrm{~W} \mathrm{~m}^{-2} \mathrm{~K}^{-4}\right), \alpha$ is the surface albedo (-), and $\varepsilon$ is the surface emissivity (-). In equation (4.2), $H$ is the sensible heat flux $\left(\mathrm{W} \mathrm{m}^{-2}\right), L E$ is the latent heat flux $\left(\mathrm{W} \mathrm{m}^{-2}\right)$, and $G_{0}$ is the soil heat flux $\left(\mathrm{W} \mathrm{m}^{-2}\right)$.

The sensible heat flux, $H$, is calculated through the bulk heat transfer equation based on the MOST (Garratt, 1994; Brutsaert, 1998):

$$
H=-\rho c_{p} C_{h} u\left[\theta_{\text {air }}-\theta_{s f c}\right]
$$

where $\rho$ is the air density $\left(\mathrm{kg} \mathrm{m}^{-3}\right), c_{p}$ is the specific heat capacity of dry air $\left(=1005 \mathrm{~J} \mathrm{~kg}^{-1} \mathrm{~K}^{-1}\right), C_{h}$ is the land-atmosphere exchange coefficient for heat $(-), u$ is the wind speed $\left(\mathrm{m} \mathrm{s}^{-1}\right), \theta_{\text {air }}$ is the potential air temperature $(\mathrm{K})$, and $\theta_{s f c}$ is the potential temperature at the surface $(\mathrm{K})$. It is worth noting that $C_{h}$ is calculated based on the roughness lengths, which will be introduced later.

In the Noah LSM, the potential evaporation ( $L E P)$ is calculated using a Penmanbased energy balance approach (Mahrt and Ek, 1984). The derivation of LEP imposes a saturated ground surface and zero canopy resistance while combining a 
bulk aerodynamic formulation with a surface energy balance expression to yield a diurnally varying $L E p$. Assuming the surface exchange coefficient for water vapor $\left(C_{q}\right)$, the diurnally dependent $L E p$ can be written as:

$$
L E_{P}=\frac{\Delta\left(R_{n e t}-G_{0}\right)+\rho \lambda C_{q} u\left(q_{s}-q\right)}{1+\Delta},
$$

where $\Delta$ is the slope of the saturated vapor pressure curve $\left(\mathrm{kPa} \mathrm{K}^{-1}\right), \lambda$ is the latent heat of vaporization $\left(\mathrm{J} \mathrm{kg}^{-1}\right) ; C_{q}$ is the exchange coefficient for water vapor; $q_{s}$ and $q$ are the saturation and actual specific humidity $\left(\mathrm{kg} \mathrm{kg}^{-1}\right)$ at the first atmospheric model level, respectively. It is worth noting that $C_{q}$ is assumed to be the same as $C_{h}$ and calculated based on the roughness lengths, which will be introduced later.

The actual evapotranspiration $(E T)$ is calculated as the sum of three components, which are soil evaporation (Edir), evaporation of intercepted precipitation by the canopy $\left(E_{c}\right)$, and transpiration through the stomata of the vegetation $\left(E_{t}\right)$. The soil evaporation extracted from the top soil layer is calculated as:

$$
E_{d i r}=\left(1-f_{c}\right)\left(\frac{\theta_{1}-\theta_{w}}{\theta_{s}-\theta_{w}}\right)^{f x} L E_{P},
$$

where $f_{c}$ is the fractional vegetation cover, $f x$ is an empirical constant taken equal to 2.0, $\theta_{s}$ is the saturated soil moisture content, $\theta_{w}$ is the soil moisture content at wilting point, and $\theta_{1}$ is the soil moisture content in the first soil layer (all in $\mathrm{m}^{3} \mathrm{~m}^{-3}$ ).

The direct evaporation of rain intercepted by the canopy is calculated as,

$$
E_{c}=f_{c} L E_{P}\left(\frac{c m c}{c m c_{\max }}\right)^{0.5},
$$

where $c m c$ and $c m c_{\text {max }}$ are the actual and maximum canopy moisture contents $\left(\mathrm{kg} \mathrm{m}^{-2}\right)$.

Moreover, the evaporation from the root zone through the stomata, often referred to as transpiration, is determined following, 


$$
E_{t}=f_{c} P_{c} L E_{P}\left[1-\left(\frac{c m c}{c m c_{\max }}\right)^{0.5}\right]
$$

where $P_{c}$ is the plant coefficient.

The soil heat flux, $G_{0}$, is calculated following Fourier's Law using the temperature gradient between the surface and the mid-point of the first soil-layer:

$$
G_{0}=K_{h}(\theta) \frac{\partial(T)}{\partial(Z)}=K_{h}(\theta) \frac{T_{s f c}-T_{s 1}}{d z},
$$

where $T_{s 1}$ is the temperature at the mid-point of the first soil layer (K), and $K_{h}$ is the soil thermal conductivity $\left(\mathrm{W} \mathrm{m}^{-1} \mathrm{~K}^{-1}\right)$ that is a function of soil water content $(\theta)$ and soil properties.

\subsubsection{Runoff simulation and water budget}

The Noah surface infiltration scheme follows a simple water balance model (Schaake et al., 1996) for its treatment of the subgrid variability of precipitation and soil moisture. Surface water is generated when the rain intensity exceeds the infiltration capacity and is calculated as:

$$
R_{\text {surf }}=P-I_{\max }
$$

where $R_{\text {surf }}$ is the surface runoff $\left(\mathrm{m} \mathrm{s}^{-1}\right), P$ is the rain intensity $\left(\mathrm{m} \mathrm{s}^{-1}\right)$, and $I_{\max }$ is the maximum infiltration capacity $\left(\mathrm{m} \mathrm{s}^{-1}\right)$.

$I_{\max }$ can be written as:

$$
I_{\max }=P \frac{D_{b}[1-\exp (k d t)]}{P+D_{b}[1-\exp (k d t)]}
$$

where $D_{b}$ is the total soil moisture deficit in the soil column $\left(\mathrm{m}^{3} \mathrm{~m}^{-3}\right)$ and $k d t$ is a constant (-) defined by, 


$$
k d t=k d t_{\text {ref }} \frac{K_{s}}{K_{r e f}}
$$

where $K_{s}$ is the saturated hydraulic conductivity $\left(\mathrm{m} \mathrm{s}^{-1}\right), k d t_{r e f}$ and $K_{\text {ref }}$ are experimentally determined parameters set to $3.0(-)$ and $2.0 \times 10^{-6}\left(\mathrm{~m} \mathrm{~s}^{-1}\right)$ for largescale simulations, respectively.

The base flow is calculated as follows:

$$
R_{\text {base }}=\operatorname{SLOPE} \cdot K\left(\theta_{4}\right)
$$

where $K$ is the hydraulic conductivity $\left(\mathrm{m} \mathrm{s}^{-1}\right), \theta_{4}$ is the moisture content in the fourth soil layer, and SLOPE is the slope coefficient (-).

The water balance equation can be written as:

$$
\begin{gathered}
P=Q+E T+\Delta S, \\
Q=R_{\text {surf }}+R_{\text {base }},
\end{gathered}
$$

where $Q$ is the runoff $\left(\mathrm{m} \mathrm{s}^{-1}\right), \quad E T$ is the evapotranspiration $\left(\mathrm{m} \mathrm{s}^{-1}\right)$, and $\Delta S\left(\mathrm{~m} \mathrm{~s}^{-1}\right)$ is the change in water storage.

\subsubsection{Roughness length parameterizations for the Noah LSM}

Z95 and C09 are two roughness length schemes which are currently utilized within the Noah LSM, and their formulations are shown in Table 4.1. In the scheme Z95, $z o m$ is defined as a function of land cover, and the Reynolds number-dependent formulation proposed by Zilitinkevich (1995) is implemented for the zoh calculation. The Zilitinkevich's coefficient $\left(C_{z i l}\right)$ is an empirical constant and currently specified as 0.1 in the Noah LSM based on calibration with field data measured over grassland (Chen et al., 1997). As can be seen from Table 4.1, $C_{z i l}$ is a key parameter for zoh calculation. However, Chen and Zhang (2009) found that the parameterization of $C_{z i l}$ in Z95 is unable to reproduce the seasonal variations of $z$ oh due to plant growth pattern, and proposed to relate $C_{z i l}$ to canopy height. Therefore, 
in the scheme C09, $C_{z i l}$ was calculated based on $z o m\left(C_{z i l}=10^{-0.4 z_{0 m} / 0.07}\right)$, whereas the seasonal values of $z 0 m$ was calculated based on GVF (Table 4.1). More specifically, the values of $z 0 \mathrm{~m}$ for grassland in $\mathrm{C} 09$ are linearly interpolated between a minimum (zom,min, equal to bare soil zom when $\mathrm{GVF}=0)$ and a maximum (zom,max, equal to fully vegetated $z$ om when $G V F=1$ ). This modification is based on a relationship derived from 12 AmeriFlux datasets collected over a variety of land covers and climate regimes.

The surface exchange coefficient for heat $\left(C_{h}\right)$ and water vapor $\left(C_{q}\right)$ transfers are parameterized as functions of roughness lengths by Chen et al. (1997) as follows:

$$
C_{h}=C_{q}=\frac{\kappa^{2} / R}{\left[\ln \left(\frac{z}{z_{0 m}}\right)-\Psi_{m}\left(\frac{z}{L}\right)+\Psi_{m}\left(\frac{z_{0 m}}{L}\right)\right]\left[\ln \left(\frac{z}{z_{0 h}}\right)-\Psi_{h}\left(\frac{z}{L}\right)+\Psi_{h}\left(\frac{z_{0 h}}{L}\right)\right]},
$$

where $z$ om is the roughness length for momentum transport (m), zoh is the roughness length for heat transport, $\Psi_{m}$ and $\Psi_{h}$ are the stability correction function for momentum and sensible heat transfer, respectively; $L$ is the Obukhov length $(\mathrm{m}), z$ is the observation height (m), $\kappa$ is the von Kármán constant (taken as 0.4 ), and $R$ is related to the turbulent Prandtl number (Pr) and taken as 1.0.

\subsubsection{Implementation}

In this study, we employed version 3.4.1 of the Noah LSM, which is freely available at the website http://www.ral.ucar.edu/research/land/technology/lsm.php. The United States Geological Survey (USGS) 30-second global 24category vegetation (land-use) data were used as the land use data. The corresponding vegetation parameters and soil hydraulic and thermal parameters are obtained from the default database of the Noah LSM. The monthly GVF database for the Noah LSM is based on the 5-yr (1985-90) Advanced Very High Resolution Radiometer (AVHRR) the Normalized Difference Vegetation Index (NDVI) products. Four soil layers with thickness of $0.1,0.3,0.6$, and $1.0 \mathrm{~m}$ are prescribed by the application of Noah in a default mode. The spin-up was completed by running the model repeatedly through 2004 until each of the variables, which include $T_{\text {sfe, }}$ runoff and soil moisture, reaches equilibrium, when 
each of the variables for the spin-up in the $n$th year meets the condition of the following equation:

$$
\left|\operatorname{Var}^{n}-\operatorname{Var}^{n-1}\right|<0.001\left|\operatorname{Var}^{n-1}\right|,
$$

where Var stands for each of the variables for the spin-up, $n$ is the year number.

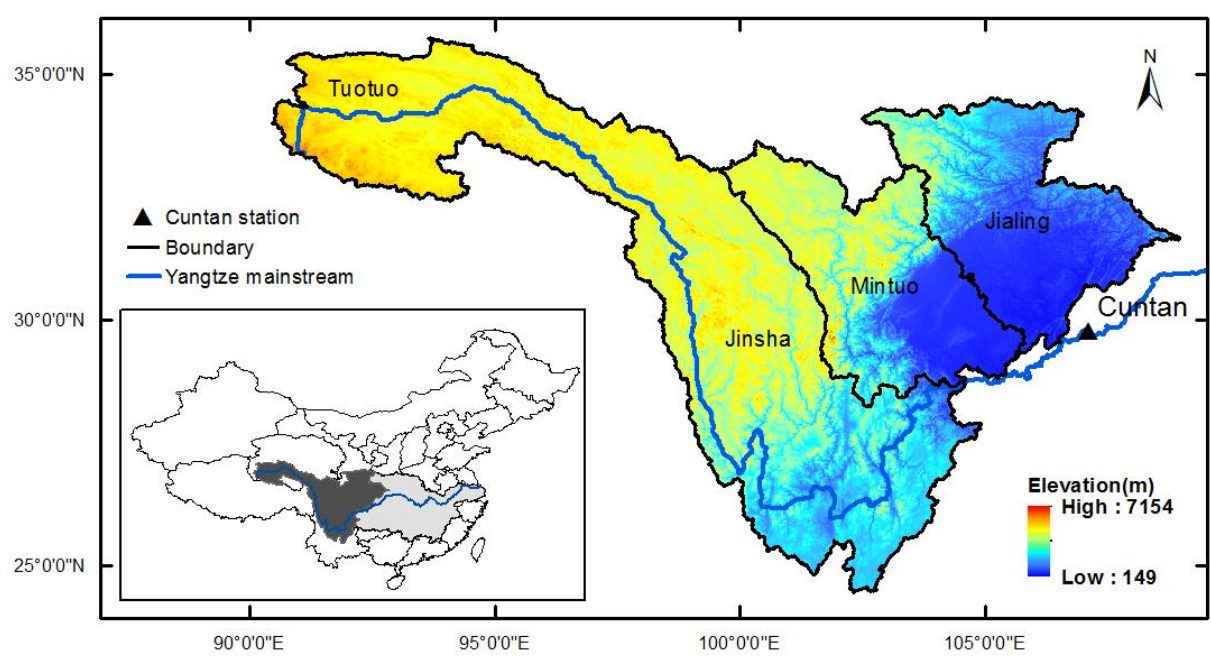

Figure 4.1. Elevation map of the Jinsha, Mintuo and Jialing sub basins located in the upper reaches of the Yangtze. The black triangle represents the Cuntan hydrological station. The main stream of the Yangtze is delineated in blue, and the boundary of the three sub basins in black.

\subsection{Study area and data processing}

The Jinsha, Mintuo and Jialing sub basins in the upper reaches of the Yangtze, located in the central and eastern part of the Tibetan Plateau, are the study area. As shown in Figure 4.1, the source region of the Yangtze River lies in a high-altitude mountainous area. The Tuotuo River is the source of the Yangtze River, and originates from the glaciers of the Jianggendiru Snow Mountains in the Tanggula Mountain Range. 


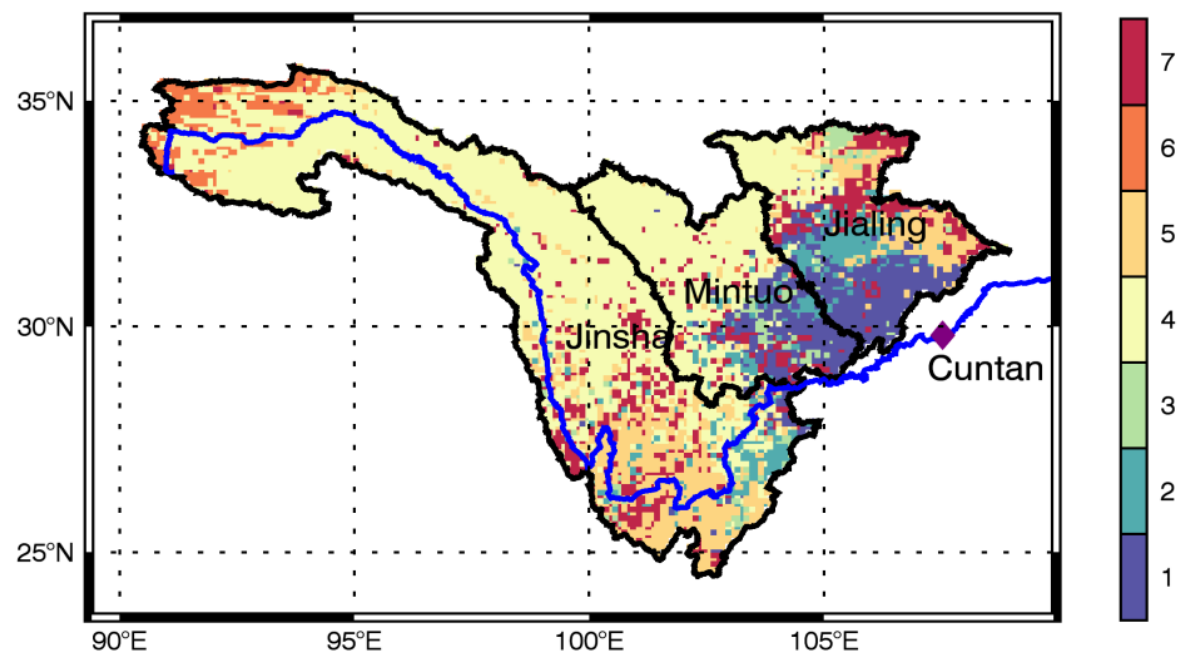

Figure 4.2. The distribution of vegetation based on the United States geological Survey (USGS) land cover classification. The classes 1 to 7 represent the land covers of the dryland cropland and pasture (1), irrigated cropland and pasture (2), cropland/grassland mosaic (3), grassland (4), shrubland (5), mixed shrubland/grassland (6), and others (7), respectively. It is noted that Class 4 represents the land cover of grassland.

The climate in the study area is governed by the East Asian monsoon. As a result, it has a large southeast-northwest precipitation gradient, and the annual precipitation (rainfall and snowfall) amount within the study area tends to decrease inland. The annual precipitation amount is about $400 \mathrm{~mm} \mathrm{yr}^{-1}$, and $85 \%$ of which occurs during the wet/warm season from May to October. This is a specific weather phenomenon of the Yangtze River basin. Under the unique plateau climate, the land cover of the study area mainly consists of grasslands (Figure 4.2). More specifically, these alpine grasslands vary from semi-arid steppe and shrub lands, to alpine steppe and moist alpine meadows, which are closely associated with the precipitation gradient across the plateau. 
Table 4.1. Experiments with different parameterizations of roughness lengths $(\mathrm{m})$ for momentum (zom) and heat $(z \circ h)$ transfer implemented in Noah LSM. $C_{z i l}$ is the Zilitinkevich (1995) empirical coefficient, $\mathrm{Re}^{*}$ is the roughness Reynolds number, $u_{*}$ is the friction velocity $\left(\mathrm{m} \mathrm{s}^{-1}\right), v$ is the kinematic molecular viscosity $\left(\right.$ taken as $\left.1.5 \times 10^{-5} \mathrm{~m}^{2} \mathrm{~s}^{-1}\right)$. GVFnorm $=\left(G V F-G V F_{\min }\right) /\left(\mathrm{GVF}_{\max }-\mathrm{GVF}_{\min }\right)$

\begin{tabular}{|c|c|c|c|}
\hline $\begin{array}{l}\text { Experiment/Pa } \\
\text { rameterization }\end{array}$ & Scheme & Formulation & Reference/Source \\
\hline Z95-original & Z95 & $\begin{array}{l}z_{0 h}=z_{0 m} \exp \left(-\kappa C_{z i l} \sqrt{\operatorname{Re} *}\right) \\
\operatorname{Re} *=u_{*} z_{0 m} / v \\
C_{z i l}=0.1 \\
z 0 m=0.120 \text { for grassland }\end{array}$ & Zilitinkevich(1995) \\
\hline Z95-updated & Z95 & $\begin{array}{l}z_{0 h}=z_{0 m} \exp \left(-\kappa C_{z i l} \sqrt{\operatorname{Re}^{*}}\right) \\
\operatorname{Re} *=u_{*} z_{0 m} / v \\
C_{z i l}=0.1 \\
z 0 m=0.035 \text { for grassland }\end{array}$ & $\begin{array}{l}\text { Zilitinkevich (1995) } \\
\text { Zheng et al. (2014) }\end{array}$ \\
\hline C09-original & $\mathrm{C} 09$ & $\begin{array}{l}z_{0 h}=z_{0 m} \exp \left(-\kappa C_{z i l} \sqrt{\operatorname{Re} *}\right) \\
\operatorname{Re} *=u_{*} z_{0 m} / v \\
C_{z i l}=10^{-0.4 z_{0 m} / 0.07} \\
z_{0 m}=\left(1-G V F_{n o r m}\right) z_{0 m, \text { min }}+G V F_{n o r m} z_{0 m, \text { max }} \\
z_{0 m, \min }=0.100, z 0 m, \max =0.120 \text { for grassland }\end{array}$ & $\begin{array}{l}\text { Chen and Zhang } \\
\text { (2009) }\end{array}$ \\
\hline C09-updated & C09 & $\begin{array}{l}z_{0 h}=z_{0 m} \exp \left(-\kappa C_{z i l} \sqrt{\operatorname{Re} *}\right) \\
\operatorname{Re} *=u_{*} z_{0 m} / v \\
C_{z i l}=10^{-0.4 z_{0 m} / 0.07} \\
z_{0 m}=\left(1-G V F_{n o r m}\right) z_{0 m, \text { min }}+G V F_{n o r m} z_{0 m, \text { max }} \\
z_{0 m, \text { min }}=0.011, z_{0 m, m a x}=0.035 \text { for grassland }\end{array}$ & $\begin{array}{l}\text { Chen and Zhang } \\
\text { (2009) } \\
\text { Zheng et al. (2014) }\end{array}$ \\
\hline
\end{tabular}

The regional accuracy of the Noah runoff output is assessed by comparing the spatially averaged time series of runoff in the study area to the corresponding time series generated from the observed discharge at the Cuntan gauging station for the study period. The procedure of computing the spatially averaged time series of runoff is based on the method of Balsamo et al. (2009) and is implemented as follows. Firstly, the discharge data $\left(\mathrm{m}^{3} \mathrm{~s}^{-1}\right)$ of the Cuntan station is accumulated to monthly discharge $\left(\mathrm{m}^{3}\right.$ month $\left.^{-1}\right)$, and divided by the area of the study area, because 
the Cuntan gauging station is the outlet of the study area. Secondly, the daily runoff data simulated by the Noah LSM are accumulated to monthly values at each pixel during the study period. The spatially averaged time series of Noah runoff is then computed as the spatial-mean of these accumulated monthly values of all pixels located in the study area.

The observed discharge data of the Cuntan station are barely influenced by significant aquifers operation or other human activities, as human activities mainly occur in the middle and lower reaches of the basin. More specifically, the Three Gorges Reservoir (TGR) has little influence on the discharge of the Cuntan station, as Cuntan forms the entrance to the TGR (Yang et al., 2010). According to the Global Map of Irrigation Areas (GMIA) provided by the global water information system (AQUASTAT) of Food and Agriculture Organization (FAO), the minority of irrigation occurs in the source region of the basin. Thus, the observed discharge of the Cuntan station is also not much affected by irrigation. In addition, the population in the source region of the basin is small, and therefore the effects of human water use on the observed discharge of the Cuntan station can be negligible.

\subsection{Experiments design}

To assess the effects of roughness length parameterizations on the Noah LSM simulations, we designed four numerical experiments with different configurations, i.e. Z95-original, Z95-updated, C09-original, and C09-updated (Table 4.1). These four experiments were conducted for the study area during the period 2005-2010. The first two experiments, Z95-original and Z95-updated, employ the Z95 scheme, whereas C09-original and C09-updated use the C09 scheme. The original Noah values of roughness lengths ( $z_{0 m}=0.12 \mathrm{~m}$ for grassland and $z_{0 m}=0.1 \mathrm{~m}$ for bare soil) are used in Z95-original and C09-original, whereas Z95-updated and C09-updated use the revised $z$ m values ( $z_{0 m}=0.035 \mathrm{~m}$ for grassland and $z_{0 m}=0.011 \mathrm{~m}$ for bare soil) by Zheng et al. (2014). The values of zom used in Z95-original and C09-original for the Noah LSM are publicly available in the vegetation parameter table, which are described at the official Noah LSM website (http://www.ral.ucar.edu/research/land/technology/lsm.php). 

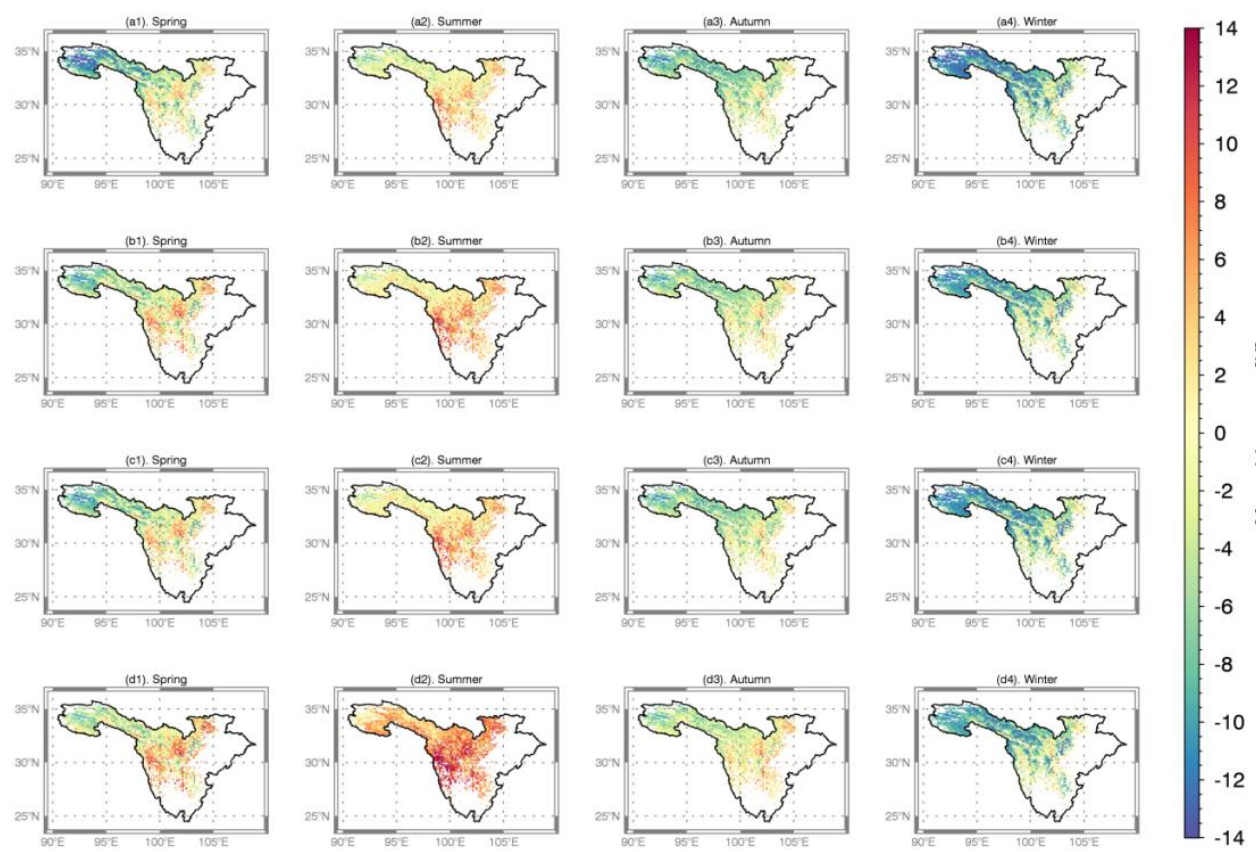

Figure 4.3. The mean bias errors of the Noah simulations at 11 A.M (local solar time) produced by Z95original $\left(\mathrm{a}^{*}\right)$, Z95-updated $\left(\mathrm{b}^{*}\right)$, C09-original $\left(\mathrm{c}^{*}\right)$, and C09-updated $\left(\mathrm{d}^{*}\right)$ during spring $\left({ }^{*} 1\right)$, summer $\left({ }^{*} 2\right)$, autumn ( $\left.{ }^{*} 3\right)$, and winter $\left({ }^{*}\right.$ ) based on 312 daytime images of MOD11C1 $T_{\text {sfc }}$ products (around 10:30 A.M, local solar time) during the period 2005-2010. Units: K.

\subsection{Results}

\subsubsection{Impacts on $T_{s f c}$ and surface energy budget modelling}

The mean bias errors (MBEs) between the Noah LSM simulations at 11 A.M (local solar time) and the selected 312 daytime images of MOD11C1 $T_{s f c}$ in the period 2005-2010 are computed for the different roughness length parameterizations and for four seasons (Figure 4.3). The histograms of the seasonal bias errors for grassland are depicted in Figure 4.4. Figures 4.3 and 4.4 both show that Z95original and C09-original generally underestimate the $T_{s f c}$ in spring, autumn and winter. Especially in winter, the $T_{\text {sf } c}$ is significantly underestimated in Z95-original and C09-original, with MBE of -7.6K (Figure 4.4(a4)) and -6.8K (Figure 4.4(c4)), respectively. These results are consistent with the previous finding that the Noah 
LSM tends to underestimate the $T_{s f c}$ during the daytime when applied to arid and semiarid regions (e.g. Hogue et al., 2005; Yang et al., 2009).
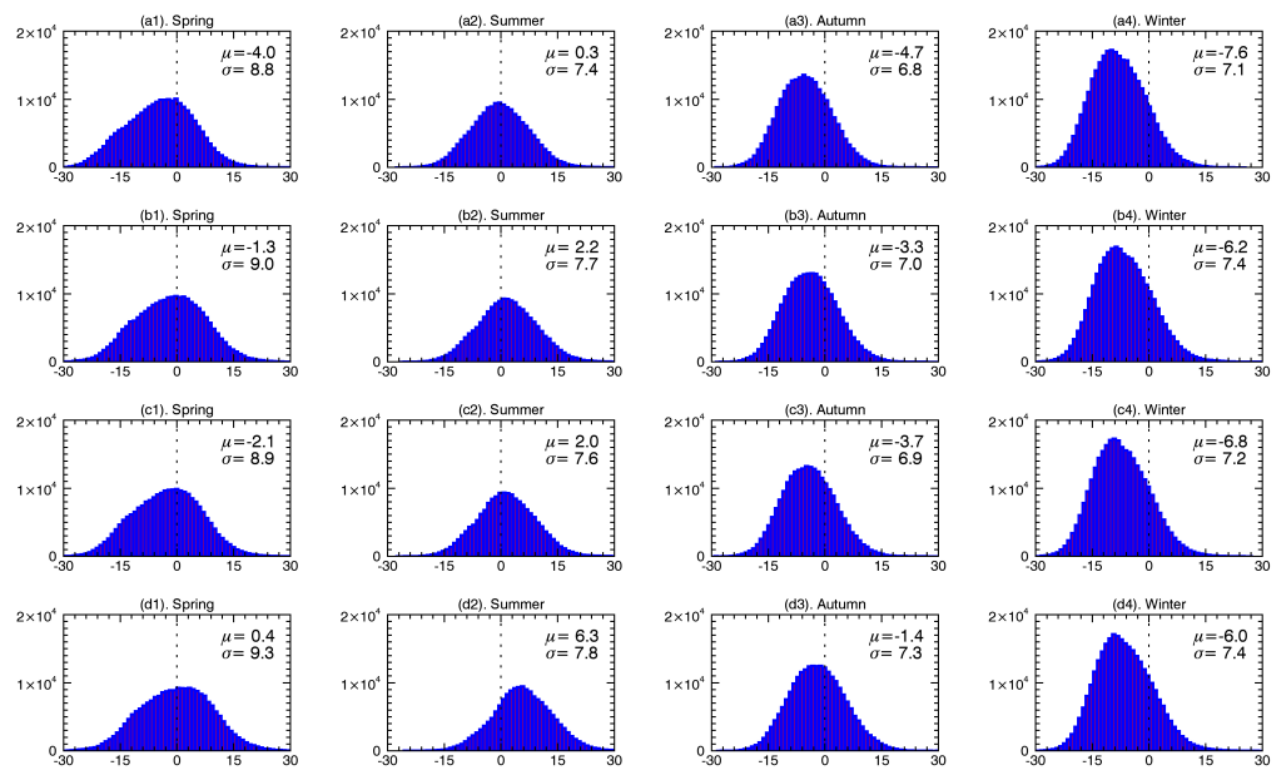

Figure 4.4. The histograms of the bias errors of the Noah simulations at 11 A.M (local solar time) produced by Z95-original $\left(\mathrm{a}^{*}\right)$, Z95-updated $\left(\mathrm{b}^{*}\right)$, C09-original $\left(\mathrm{c}^{*}\right)$, and C09-updated $\left(\mathrm{d}^{*}\right)$ during spring $\left({ }^{*} 1\right)$, summer $\left({ }^{*} 2\right)$, autumn $\left({ }^{*} 3\right)$, and winter $\left({ }^{*} 4\right)$ based on 312 daytime images of MOD11C1 $T_{\text {sfc }}$ products (around 10:30 A.M, local solar time) during the period 2005-2010. $\mu$ and $\sigma$ respectively represent the mean value and the standard deviation of the histograms. Units: $\mathrm{K}$.

On average, Z95-updated reduces the MBEs relative to Z95-original by 2.7, 1.4 and $1.4 \mathrm{~K}$ for spring, autumn and winter, whereas the MBE in summer has increased by $1.9 \mathrm{~K}$. Similarly, C09-updated produces less MBE values (1.7, 2.3 and $0.8 \mathrm{~K}$ ) than C09-original in spring, autumn and winter, whereas larger MBE $(4.3 \mathrm{~K})$ is produced in summer. The use of Z95-updated and C09-updated has improved the spatial representativeness of the Noah LSM simulated $T_{s f c}$ in the relatively highaltitude regions, compared to the underestimated $T_{s f c}$ values when using Z95orignal and C09-original. The relationship between the elevation and the quality of the results on $T_{\text {sfc }}$ will be discussed later. 
Table 4.2. Means $(\mu)$ and standard deviations $(\sigma)$ of $H, R_{n e t}, L E$, and Go simulated by Z95-original, Z95updated, C09-original, and C09-updated which correspond to the evaluated $T_{s f c}$ during different seasons. Units: $\mathrm{W} \mathrm{m}^{-2}$.

\begin{tabular}{|c|c|c|c|c|c|c|c|c|c|}
\hline & \multicolumn{2}{|c|}{ Spring } & \multicolumn{2}{|c|}{ Summer } & \multicolumn{2}{|c|}{ Autumn } & \multicolumn{2}{|c|}{ Winter } \\
\hline & & $\mu$ & $\sigma$ & $\mu$ & $\sigma$ & $\mu$ & $\sigma$ & $\mu$ & $\sigma$ \\
\hline \multirow{4}{*}{$H$} & Z95-original & 159 & 79 & 110 & 62 & 80 & 47 & 105 & 51 \\
\hline & Z95-updated & 103 & 60 & 89 & 50 & 55 & 33 & 59 & 36 \\
\hline & C09-original & 117 & 60 & 92 & 48 & 60 & 34 & 71 & 39 \\
\hline & C09-updated & 70 & 40 & 67 & 33 & 39 & 21 & 35 & 22 \\
\hline \multirow{4}{*}{$R_{n e t}$} & Z95-original & 399 & 127 & 450 & 135 & 288 & 121 & 221 & 79 \\
\hline & Z95-updated & 381 & 128 & 440 & 133 & 279 & 122 & 202 & 82 \\
\hline & C09-original & 387 & 127 & 442 & 133 & 281 & 122 & 208 & 81 \\
\hline & C09-updated & 371 & 125 & 421 & 128 & 272 & 117 & 191 & 79 \\
\hline \multirow{4}{*}{$L E$} & Z95-original & 66 & 62 & 197 & 84 & 87 & 66 & 16 & 19 \\
\hline & Z95-updated & 67 & 57 & 190 & 80 & 84 & 64 & 18 & 19 \\
\hline & C09-original & 70 & 58 & 194 & 81 & 88 & 64 & 20 & 20 \\
\hline & C09-updated & 71 & 51 & 175 & 72 & 81 & 57 & 24 & 19 \\
\hline \multirow{4}{*}{$G_{0}$} & Z95-original & 175 & 74 & 144 & 67 & 121 & 63 & 100 & 45 \\
\hline & Z95-updated & 212 & 90 & 164 & 74 & 140 & 70 & 125 & 60 \\
\hline & C09-original & 200 & 83 & 158 & 71 & 132 & 68 & 117 & 54 \\
\hline & C09-updated & 232 & 94 & 183 & 77 & 152 & 72 & 131 & 63 \\
\hline
\end{tabular}

Table 4.2 lists the means $(\mu)$ and the standard deviations $(\sigma)$ of $H, R_{n e t,} L E$, and $G_{0}$ simulated by the four experiments which correspond to the evaluated $T_{s f c}$ during different seasons. The mean values of $H$ from Z95-original and C09-original are respectively much larger than those from Z95-updated and C09-updated. In other words, Z95-updated and C09-updated notably reduce $H$ values by $21 \sim 56 \mathrm{~W} \mathrm{~m}^{-2}$ compared to Z95-original and C09-original for all seasons. The standard deviations of $H$ indicate that the shapes for $H$ distribution from Z95-updated and C09updated become sharper and narrower than those of Z95-original and C09-original. As can also be seen from Table 4.2, Z95-updated and C09-updated produce averages of $R_{n e t}$ that are respectively about $9 \sim 21 \mathrm{~W} \mathrm{~m}^{-2}$ lower than Z95-original and C09-original, whereas Z95-updated and C09-updated produce averages of Go that 
are 14 37 $\mathrm{W} \mathrm{m}^{-2}$ higher than Z95-original and C09-original. The mean values of $L E$ produced by Z95-updated and C09-updated are very close to those simulated by Z95-original and C09-original, especially during spring and winter, and the differences between the $H$ produced by the four experiments are notably larger than those between the $L E$ and $G$. This is not surprising because in the dry season the conditions (e.g. soil moisture and temperature) for the production of latent heat are not favorable, and the surface energy budget is dominated by $H$ (Chen et al., 2011).

Table 4.3. MBE, R2, RMSE, RE and NSE between the observed and estimated runoff produced by Z95original, Z95-updated, C09-original, and C09-updated for the period 2005-2010.

\begin{tabular}{cccccc}
\hline Experiment & $\begin{array}{c}\text { MBE(mm } \\
\left.\text { month }^{-1}\right)\end{array}$ & $\mathrm{R}^{2}$ & $\begin{array}{c}\text { RMSE(mm } \\
\left.\text { month }^{-1}\right)\end{array}$ & $\begin{array}{c}\text { RE(mm } \\
\left.\text { month }^{-1}\right)\end{array}$ & NSE \\
\hline Z95-original & -14.12 & 0.79 & 18.09 & -0.51 & 0.38 \\
Z95-updated & -12.72 & 0.80 & 16.61 & -0.47 & 0.48 \\
C09-original & -11.06 & 0.82 & 14.96 & -0.42 & 0.58 \\
C09-updated & -8.34 & 0.83 & 12.75 & -0.34 & 0.69 \\
\hline
\end{tabular}

\subsubsection{Impacts on water budget modelling}

The agreement between the simulations produced by numerical experiments and the observations is quantified using the following statistics: MBE, coefficient of determination $\left(\mathrm{R}^{2}\right)$, root-mean-square error (RMSE), relative error (RE) and NashSutcliffe model efficiency coefficient (NSE) (Nash and Sutcliffe, 1970). NSE is commonly used to quantitatively describe the accuracy of hydrological model outputs, with a range from $-\infty$ to 1 , the closer to 1 the more accurate the model prediction (Moriasi et al., 2007). 

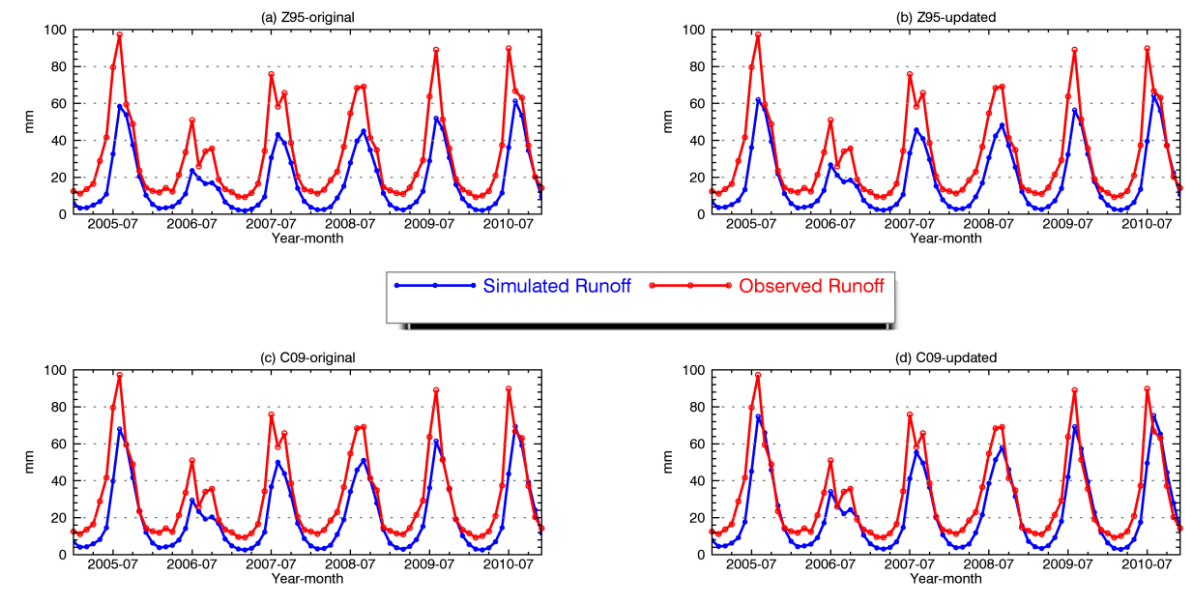

Figure 4.5. Monthly time series of the observed and simulated runoff produced by Z95-original (a), Z95updated (b), C09-original (c), and C09-updated (d) during the period 2005-2010.

Figure 4.5 compares the observed runoff with the simulation produced by all experiments. All designed experiments are capable of capturing the observed temporal pattern of the runoff, most notably the extreme drought in 2006. In comparison to measured values, the Noah LSM with Z95-original and C09-original systematically underestimated runoff, especially during the warm season (MayOctober). However, as can be seen in Figure 4.5 and Table 4.3, the Noah LSM with Z95-updated and C09-updated performs better on monthly runoff simulations than that with Z95-original and C09-original, respectively. We can also see from Figure 4.5 that the Noah LSM using C09 performs better on monthly runoff simulations than using Z95. In terms of NSE, the runoff simulations of the Noah LSM can be improved by $26.3 \%$ and $19.0 \%$ with Z95-updated and C09-updated compared to Z95-original and C09-orginal, and the runoff results produced by C09-updated have $81.6 \%, 43.7 \%$, and $19.0 \%$ improvement on that of Z95-original, Z95-updated, and C09-original, respectively. The results show that the selection of appropriate roughness length parameterizations improves the accuracy of runoff products of the Noah LSM. 

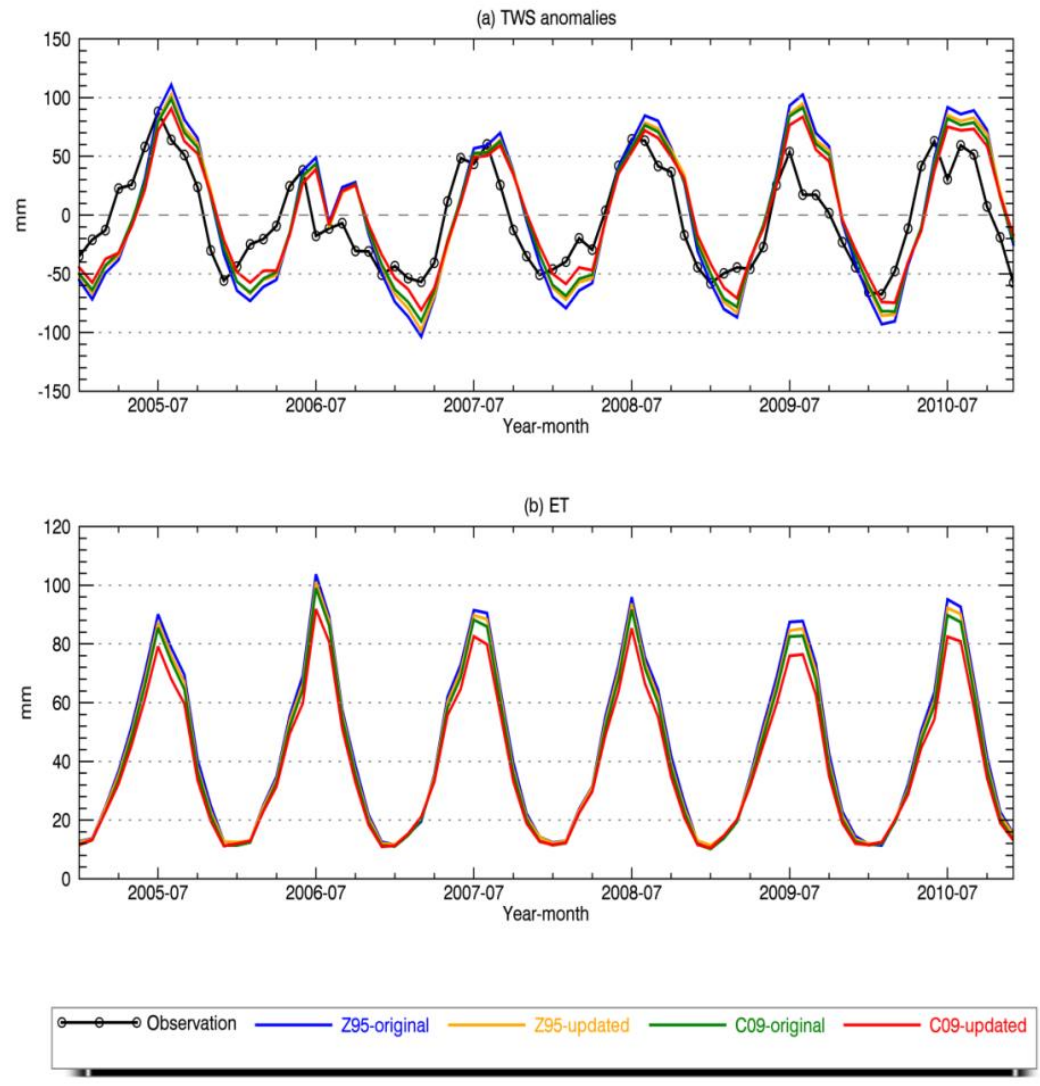

Figure 4.6. Spatially averaged monthly time series of (a) the GRACE-observed TWS anomalies and (b) ET produced by Z95-original (blue), Z95-updated (orange), C09-original (green), and C09-updated (red) during the period 2005-2010.

We further verify the TWS anomalies simulated by the different experiments with the GRACE-observed TWS anomalies. As shown in Figure 4.6(a) and Table 4.4, the seasonal variations of the TWS from Z95-updated and C09-updated better match GRACE-observed TWS variations than those obtained from Z95-original and C09orginal in terms of NSE. Similar to runoff, the simulations of TWS anomalies are improved by implementing the scheme C09 in the Noah LSM in comparison with Z95. This further demonstrates the importance of adequate roughness length parameterizations for modelling the water budget. 
Table 4.4. MBE, R², RMSE, RE and NSE between the GRACE-observed and estimated TWS anomalies produced by Z95-original, Z95-updated, C09-original, and C09-updated for the period 2005-2010.

\begin{tabular}{lccccc}
\hline Experiments & $\begin{array}{c}\text { MBE(mm } \\
\text { month-1) }\end{array}$ & $\mathrm{R}^{2}$ & $\begin{array}{c}\text { RMSE(mm } \\
\left.\text { month }^{-1}\right)\end{array}$ & $\begin{array}{c}\text { RE(mm } \\
\left.\text { month }^{-1}\right)\end{array}$ & NSE \\
\hline Z95-original & 2.38 & 0.66 & 36.19 & 0.72 & 0.23 \\
Z95-updated & 3.01 & 0.63 & 33.91 & 0.57 & 0.32 \\
C09-original & 3.13 & 0.65 & 31.71 & 0.52 & 0.41 \\
C09-updated & 3.88 & 0.63 & 30.03 & 0.33 & 0.47 \\
\hline
\end{tabular}

\subsection{Discussion}

Equation (4.15) predicts that the values of $z o m$ and $z$ oh determine the surface exchange coefficient for heat transfer, $C_{h}$, which governs the total surface heat fluxes and affects $T_{s f c}$ simulations. In the Z95 scheme, $z$ om and $C_{z i l}$ are set to constant values, whereas zoh was described as a function of $z o m$ and atmospheric conditions (e.g. wind speed). On the other hand, the $\mathrm{C} 09$ scheme estimates $z 0 m$ as a function of GVF, and both $C_{z i l}$ and zoh vary with zom. This means that the dynamic of GVF greatly affects surface heat fluxes and $T_{\text {sfc }}$ simulations when using the $\mathrm{C} 09$ scheme. The decrease of $z$ om leads to the increase of $C_{z i l}$, which will further reduce $z$ oh and increase the heat transfer resistance, which is also shown in Table 4.5.

Table 4.5. Averaged $z$ m, zoh and $C_{z i l}$ produced by Z95-original, Z95-updated, C09-original, and C09updated which correspond to the evaluated $T_{s f c}$ during different seasons.

\begin{tabular}{|c|c|c|c|c|c|c|c|c|c|c|c|c|}
\hline & \multicolumn{3}{|c|}{ Spring } & \multicolumn{3}{|c|}{ Summer } & \multicolumn{3}{|c|}{ Autumn } & \multicolumn{3}{|c|}{ Winter } \\
\hline & $z 0 m$ & $z 0 h$ & $C_{z i l}$ & $z 0 m$ & $z 0 h$ & $C_{z i l}$ & $z 0 m$ & $z 0 h$ & $C_{z i l}$ & $z 0 m$ & $z 0 h$ & $C_{z i l}$ \\
\hline Z95-original & 0.12 & 0.10 & 0.10 & 0.12 & 0.12 & 0.10 & 0.12 & 0.11 & 0.10 & 0.12 & 0.10 & 0.10 \\
\hline Z95-updated & 0.04 & 0.01 & 0.10 & 0.04 & 0.03 & 0.10 & 0.04 & 0.02 & 0.10 & 0.04 & 0.01 & 0.10 \\
\hline C09-original & 0.10 & 0.10 & 0.26 & 0.10 & 0.10 & 0.26 & 0.11 & 0.11 & 0.24 & 0.10 & 0.10 & 0.26 \\
\hline C09-updated & 0.01 & 0.01 & 0.84 & 0.03 & 0.03 & 0.67 & 0.02 & 0.02 & 0.76 & 0.01 & 0.01 & 0.85 \\
\hline
\end{tabular}



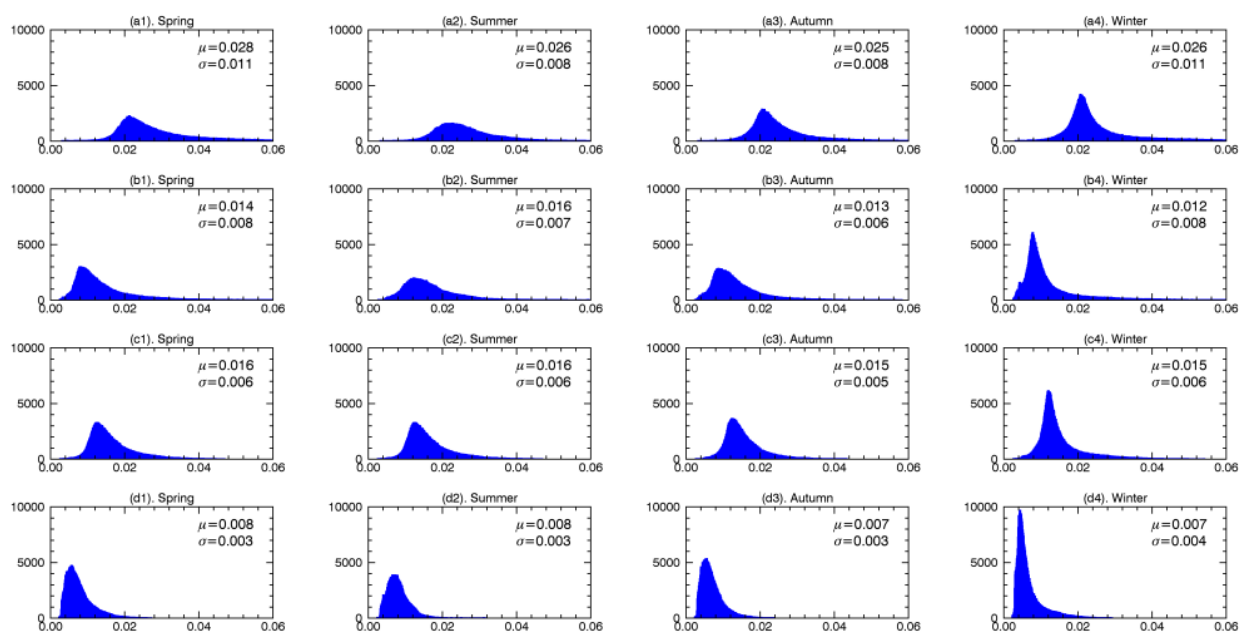

Figure 4.7. The histograms of $C_{h}$ corresponding to the evaluated $T_{\text {sfc }}$ simulated by Z95-original $\left(\mathrm{a}^{*}\right)$, Z95updated $\left(\mathrm{b}^{*}\right)$, C09-original $\left(\mathrm{c}^{*}\right)$, and C09-updated $\left(\mathrm{d}^{*}\right)$ during spring $\left({ }^{*} 1\right)$, summer $\left({ }^{*} 2\right)$, autumn $\left({ }^{*} 3\right)$, and winter $\left({ }^{*}\right)$.

The differences between the $C_{h}$ produced by the four experiments in Figure 4.7 are consistent with that of the simulated $T_{\text {sfc }}$ as shown in Figures 4.3 and 4.4, and surface heat fluxes $\left(H, R_{n e t}, L E, G\right)$ as listed in Table 4.2. More specifically, the usage of Z95-original and C09-original in the Noah LSM generally underestimates the $T_{s f c}$ for alpine grasslands in the study area in spring, autumn and winter. This indicates that the coupling of heat from the grasslands to the atmosphere is too strong, i.e. the values of $C_{h}$ are overestimated. The overestimation of $C_{h}$ will pump more $H$ to heat the atmosphere and cool down the land surface. The end result of this cooling leads to lower upward longwave radiation (thus larger $R_{n e t}$ ) and a lower soil temperature gradient (thus lower Go). On the other hand, using Z95updated/C09-updated in the Noah LSM produces smaller values of $C_{h}$ (Figure 4.7), and therefore simulates higher $T_{\text {sf }}$ compared with using Z95-original/C09-original. As a result, the performance of the Noah LSM can be generally improved during spring, autumn, and winter. In contrast, using Z95-updated/C09-updated in the Noah LSM increases the warm biases in summer, when the $T_{\text {sfc }}$ simulation results from the Noah LSM with Z95-original/C09-orignal match well with the $T_{\text {sfc }}$ of MODIS. 

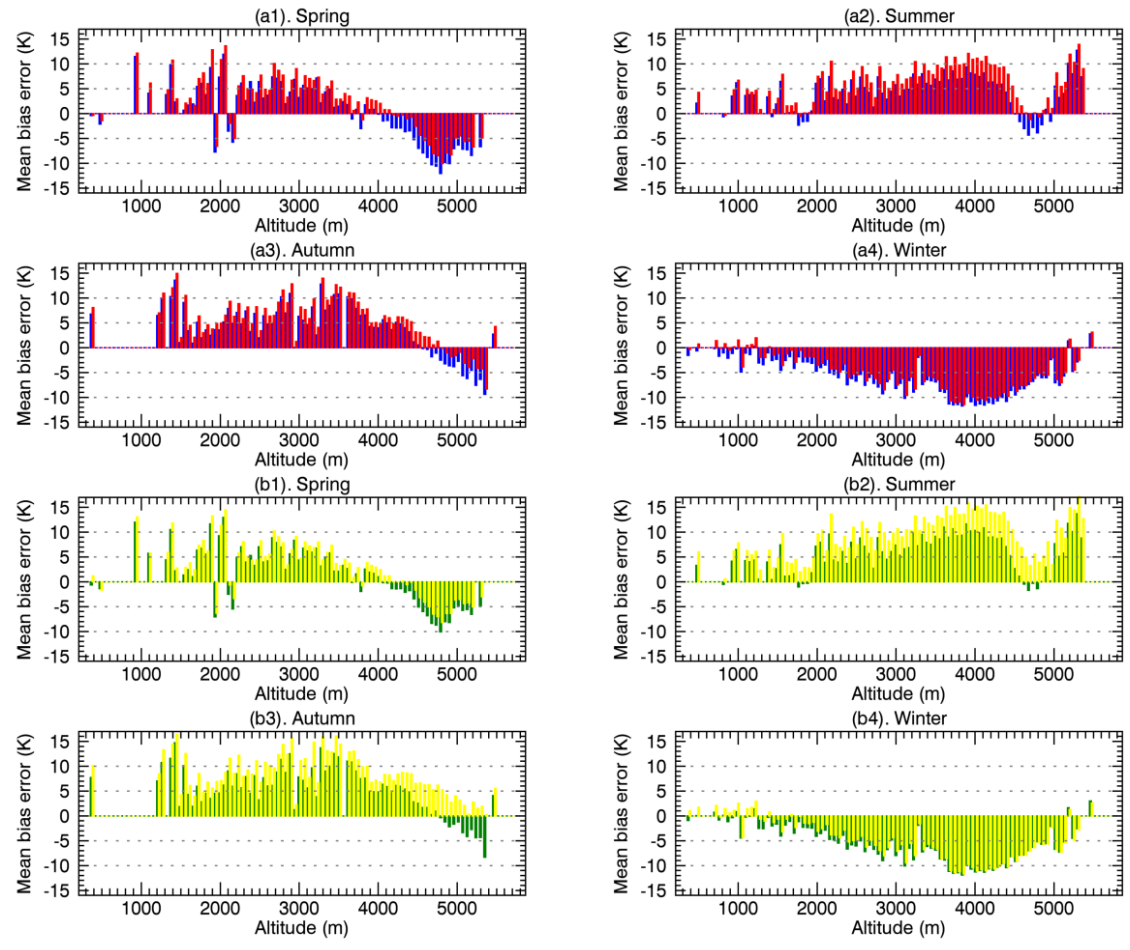

Z95-original

Z95-updated

C09-original Co9-updated

Figure 4.8. The mean bias errors of the Noah simulations for different altitudes at 11 A.M (local solar time) produced by Z95-original (blue), Z95-updated (red), C09-original (green), and C09-updated (yellow) during spring $\left({ }^{*} 1\right)$, summer $\left({ }^{*}\right)$, autumn $\left({ }^{*} 3\right)$, and winter $\left({ }^{*} 4\right)$ based on 312 daytime images of MOD11C1 $T_{s f c}$ products (around 10:30 A.M, local solar time) during the period 2005-2010. Units: K.

The reason that the Noah LSM performs differently in summer is the distinct seasonal march of the surface water and energy budget in the central and eastern Tibetan Plateau. During the monsoon period (usually from June to September), the dry land surface becomes wet due to frequent rainfall events, and hence LE dominates the energy budget instead of $H$ (Yang et al., 2008). This demonstrates that the Noah LSM with Z95-orignal/C09-orignal can perform well on $T_{\text {sfc }}$ simulations for alpine grasslands during wet monsoon seasons, which is consistent with the assessment of the Noah LSM on the $T_{\text {sf }}$ simulations using observations in the Tibetan Plateau (Yang et al., 2008). This also implies that the schemes Z95 and C09 do not produce the seasonal variations of the $T_{s f}$ simulations in the Tibetan Plateau. We can also see from Figures 4.3 and 4.4 that the Noah LSM using the C09 
scheme generally performs better for grasslands than that using the scheme Z95. This is because the scheme Z95 tends to largely underestimate the heat transfer resistances for short vegetation (grassland, crops, shrub) in dry conditions (e.g. Chen et al., 2011), and relating $C_{h}$ to the GVF dynamic can more realistically represent surface exchange processes in LSMs (Chen and Zhang, 2009).

As shown in Figure 4.8, the Noah LSM with Z95-updated/C09-updated improves the $T_{s f c}$ simulation performance in the relatively high-altitude regions (above 4000 $\mathrm{m})$, which may suggest a relation between the Noah LSM of $T_{\text {sf }}$ and the elevation. This relationship was also confirmed by, for instance, Salama et al. (2012). This dependency is mainly attributed to the fact that the climatology and land cover conditions of the higher altitude central Tibetan Plateau are different from the lower elevation southeastern Tibetan Plateau (Figure 4.1). More specifically, due to the large southeast-northwest precipitation gradient of the Tibetan Plateau (ranging from 100 to $700 \mathrm{~mm}$ in mean annual precipitation), the southeastern plateau is relatively wetter than the central part (Yang et al., 2008). As a result, the distribution of the Tibetan grasslands is closely associated with the precipitation gradient across the plateau. The central plateau mainly consists of semi-arid steppe, whereas moist alpine meadow occurs in the relatively mesic southeastern plateau (Yang et al., 2010; Su et al., 2013). This further adds weight to the notion that the usage of Z95-updated and C09-updated is more suitable under dry conditions. It also indicates that the roughness length schemes Z95 and C09 have difficulties in estimating the reliable spatial distribution of daytime $T_{s f c}$ for a region that has diverse climate and land surface conditions. Besides, this suggests that the vegetation types presented on the land surface in the Noah LSM should be further specified with respect to hydro-meteorological conditions, and the roughness length parameterizations should be selected and, if needed, modified according to these specified vegetation types. The grassland in the Tibetan Plateau, for instance, can be classified into arid alpine steppe, semi-arid alpine steppe, moist alpine meadows, etc.

The standard deviations of the four experiments for the same season (shown in Figure 4.4) are very close, indicating that changing the roughness lengths only changes MBE without affecting the shape of the probability distribution of the errors. On the one hand, it means that the MBE can be used as the single indicator 
for the evaluation of the simulated $T_{\text {sf. }}$. On the other hand, our results show that it is possible to model the probability distribution of the errors between Noah simulation and MODIS satellite observation, which can be used for data assimilation.

Besides the $T_{s f c}$ and surface heat fluxes simulations, the roughness length parameterizations have large effects on the simulations of the water budget components. As described in section 4.6, Z95-updated/C09-updated improves the monthly runoff and TWS simulations (Figures 4.5 and 4.6), which indicates that a more realistic representation of the heat transfer resistance can increase the Noah LSM simulation accuracy of the water fluxes and states. The improvement in runoff simulation is attributed to the increased amount of monthly runoff (Figure 4.5 and Table 4.3), predominantly the increase of base flow (Figure 4.9).

The fact that the Noah LSM with Z95-updated/C09-updated increases the monthly amount of base flow and benefits the TWS anomalies can be explained as follows. The higher $T_{\text {sf }}$ simulated by the Noah LSM with Z95-updated/C09-updated, due to the decreased $C_{q}$, will reduce the $R_{\text {net }}$ (equation (4.1)), lead to more $G_{0}$ by raising the ground-soil temperature gradient (equation (4.8)), and consequently reduce the LEP (equation (4.4)). Moreover, the lower zom means the decreased $C_{q}$ (equation (4.15)), which will weaken the land-atmospheric coupling strength for momentum transfer and reduce the $L E P$ (equation (4.4)). This decreased $L E P$ could reduce the actual $E T$ (equations (4.5) - (4.7)), which is consistent with that, as shown in Figure 4.6(b), the ET results of Z95-updated/C09-updated during the warm season are lower than that of Z95-original/C09-original. As ET decreases, less water is extracted from soil for $E T$, and more water is retained in the soil columns. As a result, the soil moisture of Z95-updated/C09-updated is higher than that of Z95-original/C09-original. The increased soil moisture favors the runoff generation, leading to an improved runoff simulation for Z95-updated/C09-updated. More specifically, the increased liquid soil moisture raises hydraulic conductivity (Campbell, 1974) and increases the soil drainage, leading to more base flow generation (equation (4.12)). 
(a) Surface runoff

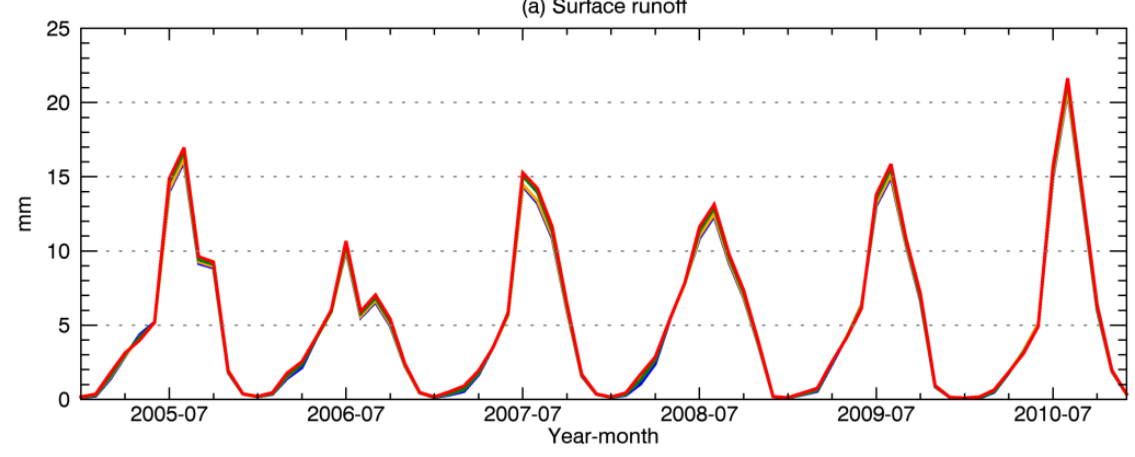

(b) Baseflow

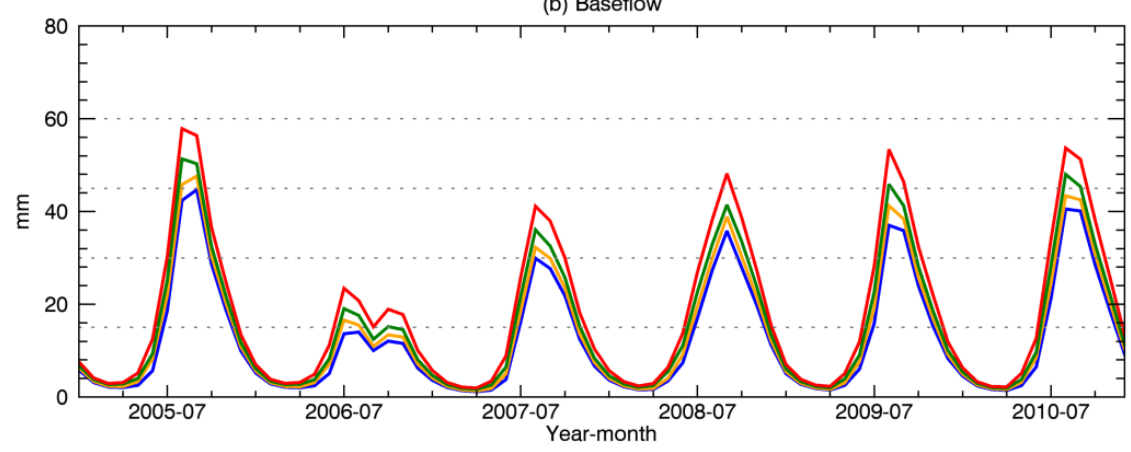

Figure 4.9. Monthly time series of (a) the surface runoff and (b) the base flow produced by Z95-original (blue), Z95-updated (orange), C09-original (green), and C09-updated (red) during the period 2005-2010.

On the other hand, we can see from Figure 4.9(a) that the increased soil moisture content has a minor effect on the simulated surface runoff. This can be attributed to the fact that the moisture deficits in the soil columns are usually small during the wet monsoon period (Schaake et al., 1996), and hence the maximum infiltration capacities calculated based on equation (4.10) for the four experiments are very close. Consequently, the surface runoff simulation, which is determined by the rain intensity and the maximum infiltration capacity as shown in equation (4.9), does not vary largely. Likewise, the difference between the amounts of runoff from Z95 
and from C09 is attributed to the difference between the values of $C_{h}\left(C_{q}\right)$ from Z95 and from $\mathrm{C} 09$. This demonstrates that the roughness length parameterizations, which determine $C_{h}$ and $C_{q}$, play an important role in controlling the total amount of runoff in the LSM simulation for high-altitude catchments.

It should be noted that the improvement in runoff simulation mainly occurs in the warm season (May-October). This is due to the fact that, during the cold season (November-April), the production of $L E$ is very low due to the limitations of, for instance, soil moisture and temperature, and therefore the roughness lengths have a negligible effect on the ET simulation. It should be noted that snow cover is limited because of strong wind and little precipitation in the study area during the cold season (Malik et al., 2014), and hence the runoff production is little influenced by the snowpack. Moreover, it may be unfair to compare the Noah LSM simulated runoff directly (without river routing) with the discharge at the gauging station. However, we compare the runoff with the observation at a monthly scale rather than an hourly or a daily scale, thus the influence of the river routing on the comparison is minor, which is supported by Yang et al. (2011) and Cai et al. (2014).

Although Z95-updated/C09-updated improves the Noah LSM simulations, the $T_{\text {sfc }}$ and monthly runoff are still largely underestimated. This can be explained by the following reasons. The first one is the imperfect roughness length schemes for high-altitude regions under frozen soil conditions. When the water in the soil is frozen, the land surfaces tend to be smoother (Zheng et al., 2014). As such, the values of $z$ oh and $z$ om are still overestimated within the Noah LSM leading to only a slight improvement in $T_{s f c}$ simulation for the winter period even when using the Z95-updated/C09-updated parameterizations (Figures 4.3 and 4.4).

The second explanation forms the inherent uncertainties associated with the simulation of the soil water flow and heat transport and their impact of the computed surface energy and water budgets. For instance, Yang et al. $(2005,2009)$ and Chen et al. (2013) have demonstrated that the absence of vertical soil heterogeneity in the model structure causes difficulties in the simulation of soil moisture and temperature profiles across the Tibetan Plateau by the Noah LSM. Furthermore, Su et al. (2013) pointed out that the simulation of freeze-thaw transitions in the soil play an important role in reliability of the modelled soil 
moisture and temperature profiles. In addition, the soil column adopted by the Noah LSM has only a $2 \mathrm{~m}$ depth, and a free gravitational drainage is employed for the base flow production. The disadvantage of this setup is the inability to redistribute water across the soil column via the capillary rise and deeper layers including groundwater (Gulden et al., 2007; Niu et al., 2011). This can lead to dryer soils, and consequently an underestimation of the runoff as Niu et al. (2005) and Yang et al. (2011) have previously reported.

The third reason is the forcing data. Inaccuracies existing in the forcing data may have substantial impacts on the land surface simulations. Chen et al. (2011) compared the daily averaged radiation fluxes of ITPCAS forcing data against that from field measurements at three dry-land sites in the Tibetan Plateau, and the results have shown that the ITPCAS data are dramatically improved in terms of radiation in comparison with the widely used GLDAS dataset, however the daily averaged longwave radiation of the ITPCAS forcing data is still notably underestimated. As incoming radiation fluxes play a key role for simulating surface energy partitioning and $T_{s f}$, the underestimated incoming radiation can be one of the reasons that cause the underestimation of $T_{\text {sff. }}$.

\subsection{Conclusions}

In this chapter, we have addressed the regional scale effects of the roughness length parameterizations for grasslands on the Tibetan Plateau in the Noah LSM. Four numerical experiments with two different roughness schemes were conducted during the period 2005-2010 for the high-altitude hydrological catchment, the source region of the Yangtze basin in China. The experimental setups were based on physical process knowledge, verified with various satellite products and validated with ground-based observations. This study highlights the need for regional adaptation of the $z_{0 m}$ and $z$ oh values, and provides insight into the regional scale land surface modelling of high-altitude catchments. The main findings of this work are listed as follows:

1). The usage of Z95-updated/C09-updated improves, validated by the MODIS products, the regional scale predictions of the Noah LSM with Z95-original/C09original on $T_{s f c}$ for a high-altitude basin in spring, autumn and winter, but larger 
warm biases are produced in summer. This implies that the roughness length schemes, Z95 and C09, cannot satisfactorily capture the seasonal variations of the $T_{\text {sfc }}$ simulations in the Tibetan Plateau, and Z95-updated and C09-updated are more suitable in relatively dry conditions.

2). Z95-updated/C09-updated improves the $T_{s f c}$ simulation performance in the relatively high-altitude regions (above $4000 \mathrm{~m}$ ), whereas larger warm biases are produced in low-altitude regions of the Tibetan Plateau. This indicates that the roughness length schemes, Z95 and C09, have difficulties in estimating the reliable spatial distribution of daytime $T_{s f c}$ for a region that has diverse climate and land surface conditions. One feasible way to address this issue is the grasslands in the Tibetan Plateau are further classified into arid alpine steppe, semi-arid alpine steppe, moist alpine meadows, etc., and the roughness length parameterizations are selected and, if needed, modified according to these further specified vegetation types.

3). The Noah LSM with Z95-original/C09-original largely underestimates the monthly runoff in the source region of the Yangtze River. However, by implementing Z95-updated/C09-updated, the monthly amount of runoff can be largely increased. Also, Z95-updated/C09-updated increases the agreement of the Noah TWS simulation with the GRACE-derived TWS. This demonstrates that the roughness length parameterization, in association with surface exchange coefficient for heat $\left(C_{h}\right)$ and moisture $\left(C_{q}\right)$ transfer, plays an important role in modelling water budget components for high-altitude catchments.

4). The Noah LSM using the scheme C09 generally performs better than that using the scheme Z95 on the simulations of regional scale $T_{\text {sfc }}$ and water budget components. It is therefore recommended to use C09-updated for LSM simulations in the central and eastern part of the Tibetan Plateau, particularly in dry conditions. 


\section{CHAPTER 5}

Reconstruction of the Yangtze River Basin Water Budget through Integration of Satellite, Ground data and Noah-MP Model Simulation 



\section{Chapter 5 Reconstruction of the Yangtze River basin water budget through integration of satellite, ground data and Noah-MP model simulations}

\section{This chapter is based on:}

Huang, Y., M. S. Salama, M. S. Krol, X. Chen, Z. Su, and A. Y. Hoekstra, 'Reconstruction of the Yangtze River basin water budget through integration of satellite, ground data and Noah-MP model simulations', in preparation for submission to Journal of Geophysical Research: Atmospheres (JGR).

\subsection{Abstract}

In this study, we evaluate the regional-scale water budget simulations by the Noah land surface model (LSM) and its augmented version, with multi-parameterization options (Noah-MP). These simulations were performed for the Yangtze River basin, and driven by the high resolution $\left(0.1^{\circ}\right)$ meteorological data developed by the Institute of Tibetan Plateau, Chinese Academy of Sciences (ITPCAS). River discharge observed at the main gauging stations of the Yangtze River is used to assess the simulated runoff. Moreover, the simulated evapotranspiration (ET) is compared to ET derived through (1) water budget closure using GRACE observed terrestrial water storage anomalies and other observations, (2) an energy budget approach using the Surface Energy Balance Systems (SEBS). Results show that a good combination of schemes of the Noah-MP LSM can significantly reduce the mean bias error (MBE) and improve the model efficiency of monthly runoff simulation. This improvement can be mainly attributed to the improved physics of supercooled liquid water, frozen soil permeability and the lower boundary condition of soil moisture. We further compared the simulations to the Global Land Data Assimilation System (GLDAS) outputs, and results indicates that the Noah-MP simulations are superior, which benefits from more accurate atmosphere forcing data and the enhanced physics from the Noah LSM. 


\subsection{Introduction}

The natural water availability can be examined by analyzing the space-time distribution of water fluxes and states, which can be obtained from publicly available global data assimilation products. Global data assimilation products such as Interim Reanalysis Data (ERA-Interim) and Global Land Data Assimilation System (GLDAS) combine the virtues of in situ data, remotely sensed observations, and land surface modeling. These products have been extensively applied in hydrological studies, and are very valuable for water resources research. However, large inaccuracy may exist at basin scales (e.g. Xue et al., 2013), which is mainly attributed to, for instance, sparse observations and model imperfection. Therefore, basin scale land surface model (LSM) simulation is a good alternative to obtain natural variability of water states and fluxes.

The inaccuracies of model simulations can be attributed to at least two main factors. The first is the uncertainties existing in forcing data, which have substantial impacts on the model results. For instance, Xue et al. (2013) evaluated the evapotranspiration (ET) in the upper Yangtze River basin reproduced using atmospheric forcing data adopted from global reanalysis, and reported that the inaccuracy of the ET estimates is mainly due to the errors in the forcing data. Another source of uncertainties is embedded within the physics adopted by the model. Each LSM utilizes a different set of parameterizations to represent the processes that govern the mass and heat exchanges at the land-atmosphere interface. For instance, Chen et al. (2011) and Zheng et al. (2014) have demonstrated, for study areas on the Tibetan Plateau, the range in performance of the Noah LSM produced with different 'state-of-the-art' roughness length parameterizations.

The developers of the Noah LSM with multi-parametrizations options (hereafter Noah-MP, Niu et al., 2011; Yang et al., 2011) acknowledge the limitation of a single parameterization of the model physics, and provide multiple alternatives for each physical process. They tested the Noah-MP at local scale and global scale for the major river basins, and demonstrated that the suit of available parametrizations within the same model framework is an efficient way to quantify the uncertainties 
induced by the model physics. Although the Yangtze River basin was taken as one of the basins for the evaluation of the Noah-MP LSM (Yang et al., 2011), only the monthly runoff climatology modeled by the Noah-MP LSM was compared with observation in terms of hydrological evaluation. Therefore, a more detailed hydrological evaluation of the Noah-MP LSM for the Yangtze River basin is necessary.

In this study, we employ the Noah and Noah-MP LSM in the High-Resolution Land Data Assimilation System (HRLDAS), which are maintained by Research Application Laboratory (RAL) of National Center for Atmospheric Research (NCAR). USA. Both models are driven by a, high resolution meteorological data $\left(0.1^{\circ} \times 0.1^{\circ}, 3\right.$-hourly $)$, which were produced by the Institute of Tibetan Plateau Research, Chinese Academy of Sciences (ITPCAS), to simulate monthly runoff and ET in the Yangtze River basin. The monthly runoff simulation during the period 2005-2010 is validated with observations at main gauging stations in the basin, and the simulated ET is compared with the ET estimates derived through (1) water budget closure using GRACE observed terrestrial water storage anomalies and other observations, (2) an energy budget approach using the Surface Energy Balance Systems (SEBS). Through this evaluation, we can investigate to what extend an appropriate selection of the enhanced physical descriptions of the NoahMP improves the simulations of water fluxes and states the Yangtze River basin.

\subsection{Noah-MP description}

Noah-MP was enhanced from the original Noah LSM through an addition of improved physics and multi-parameterization options (Niu et al., 2011; Yang et al., 2011). It separates the vegetation canopy from the ground surface rather than treating the surface layer as a bulk layer as the Noah LSM does, and introduces a semitile scheme to represent land surface heterogeneity, which computes shortwave radiation transfer through a modified two-stream radiation transfer scheme (Yang and Friedl, 2003; Niu and Yang, 2004) considering the 3-D structure of the canopy. A Ball-Berry type stomatal resistance scheme (Ball et al., 1987; Collatz et al., 1991, 1992; Sellers et al., 1996; Bonan, 1996) that related to photosynthesis and a short-term leaf dynamic model are available in Noah-MP. Moreover, a physically based three-layer snow model (Yang and Niu, 2003), a 
frozen soil scheme that produces a greater soil permeability (Niu and Yang, 2006), and a simple groundwater model with a TOPMODEL-based runoff scheme (Niu et al., 2005, 2007) were integrated into the Noah LSM.

\subsection{Numerical experiments}

As described in Table 5.1, one numerical experiment with the Noah LSM version 3 (hereafter Noah V3) and six experiments with different combinations of optional schemes of the Noah-MP LSM were designed and conducted in the study area (see Appendix A and B for details). We selected the same scheme options for all the six numerical experiments of Noah-MP, which are: (1) using Noah type for soil moisture factor for stomatal resistance, (2) using a modified two-stream approximation for radiation transfer, (3) using CLASS type for ground snow surface albedo, (4) using Jordan's (1991) scheme for partitioning precipitation into rainfall and snowfall, (5) using original Noah type for lower boundary condition of soil temperature, (6) using semi-implicit method for snow/soil temperature time scheme, (7) using the Ball-Berry model for canopy stomatal resistance, (8) using a process-based dynamic leaf model.

All the experiments were run for 21 years (1990-2010) driven by ITPCAS data set at $0.1^{\circ}$ spatial and 3-hourly temporal resolution. All the experiments were initialized by the same GLDAS-Noah LSM L4 3 hourly $0.25 \times 0.25$ degree Version 2.0 data at UTC zero time on 1990-09-01. The model outputs during the period 2005-2010 were selected for analysis and evaluation, in order to match the period over which observational runoff data are available. The period 1990-2004 was used as the spinup time, and the spin-up was completed by running the Noah and Noah-MP LSM repeatedly through this period until each of the variables (i.e. runoff, ET and soil moisture (SM)) reaches equilibrium. The same soil type and vegetation type data sets were used in these experiments. Moreover, all the experiments used the same vegetation parameters and soil hydraulic and thermal parameters, which are obtained from the default database of HRLDAS. 
Table 5.1. Experiments with Different Combinations of Schemes a

\begin{tabular}{lcccc}
\hline & $\begin{array}{c}\text { Supercooled } \\
\text { liquid water }\end{array}$ & $\begin{array}{c}\text { Frozen soil } \\
\text { permeability }\end{array}$ & Runoff & $C_{h}$ \\
\hline Noah V3 & Koren99 & Koren99 & Schaake96 & Chen97 \\
EXP 1 & Koren99 & Koren99 & Schaake96 & Chen97 \\
EXP 2 & NY06 & NY06 & Schaake96 & Chen97 \\
EXP 3 & NY06 & NY06 & Schaake96 & M-O \\
EXP 4 & Koren99 & Koren99 & SIMGM & Chen97 \\
EXP 5 & NY06 & NY06 & SIMGM & Chen97 \\
EXP 6 & NY06 & NY06 & SIMGM & M-O \\
\hline
\end{tabular}

aKoren99, Koren et al. (1999); NY06, Niu and Yang (2006); Chen97, Chen et al. (1997);

Schaake96, Schaake et al. (1996).

${ }^{\mathrm{b}}$ Although using the same selected processes here, EXP1 differs from the Noah LSM in many other aspects, such as radiation schemes, sensible and latent heat flux formulations, the skin temperature solution, and the canopy stomatal resistance calculation.

\subsection{Results and discussion}

The agreement between the simulations produced by numerical experiments and the observations is quantified using the following statistics: the mean bias error (MBE), the coefficient of determination $\left(\mathrm{R}^{2}\right)$, the root mean square error (RMSE), and the Nash-Sutcliffe efficiency (NSE) for runoff (Nash and Sutcliffe, 1970). NSE is commonly used to quantitatively describe the accuracy of hydrological model outputs, with a range from $-\infty$ to 1 , the closer to 1 the more accurate the model prediction (Moriasi et al., 2007). A Taylor diagram is also used to concisely demonstrate how well patterns of observed and modeled runoff and ET match each other in terms of their correlation, their root-mean-square (RMS) difference and the ratio of their variance (Taylor, 2001). The centered RMS difference between the simulated and observed patterns is proportional to the distance to the point on the x-axis identified as 'observed', and the normalized standard deviation of the simulated pattern is proportional to the radial distance from the origin. 

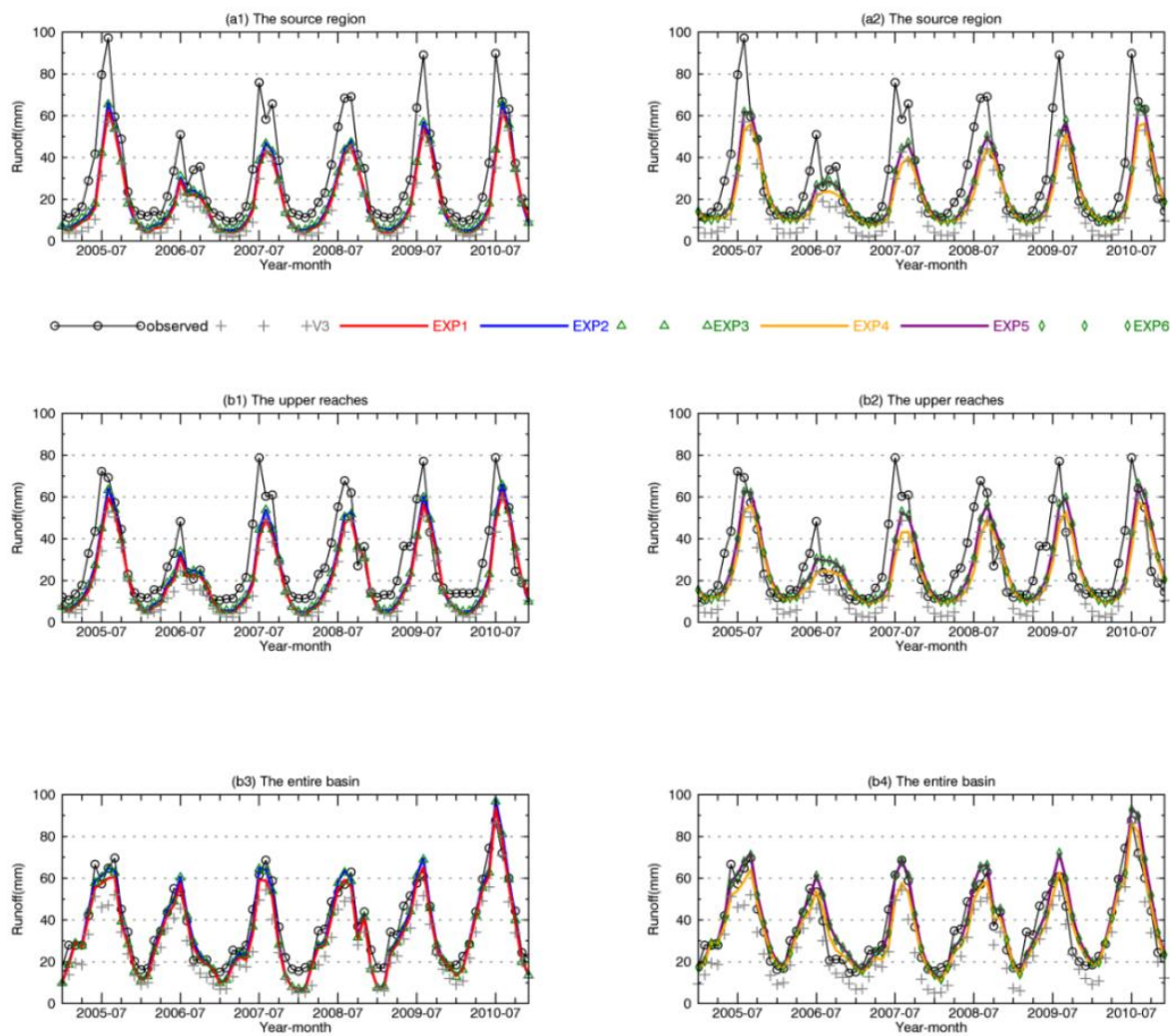

Figure 5.1. Observed, Noah V3 simulated, and EXP1-EXP6 produced monthly runoff during the period 2005-2010 for the source region, upper reaches, and the entire Yangtze River basin, respectively.

\subsubsection{Runoff}

Figure 5.1 compares the observed runoff and simulated runoff produced by the Noah LSM, and the six experiments of the Noah-MP LSM in the source region, the upper reaches, and the entire basin, respectively. The Noah LSM systematically underestimates monthly runoff, especially in the Yangtze source region. EXP1 produced runoff is slightly better than the Noah LSM, indicating that the unselected processes, for which the Noah-MP LSM differs from the Noah LSM, slightly enhance the runoff simulation in the basin. 

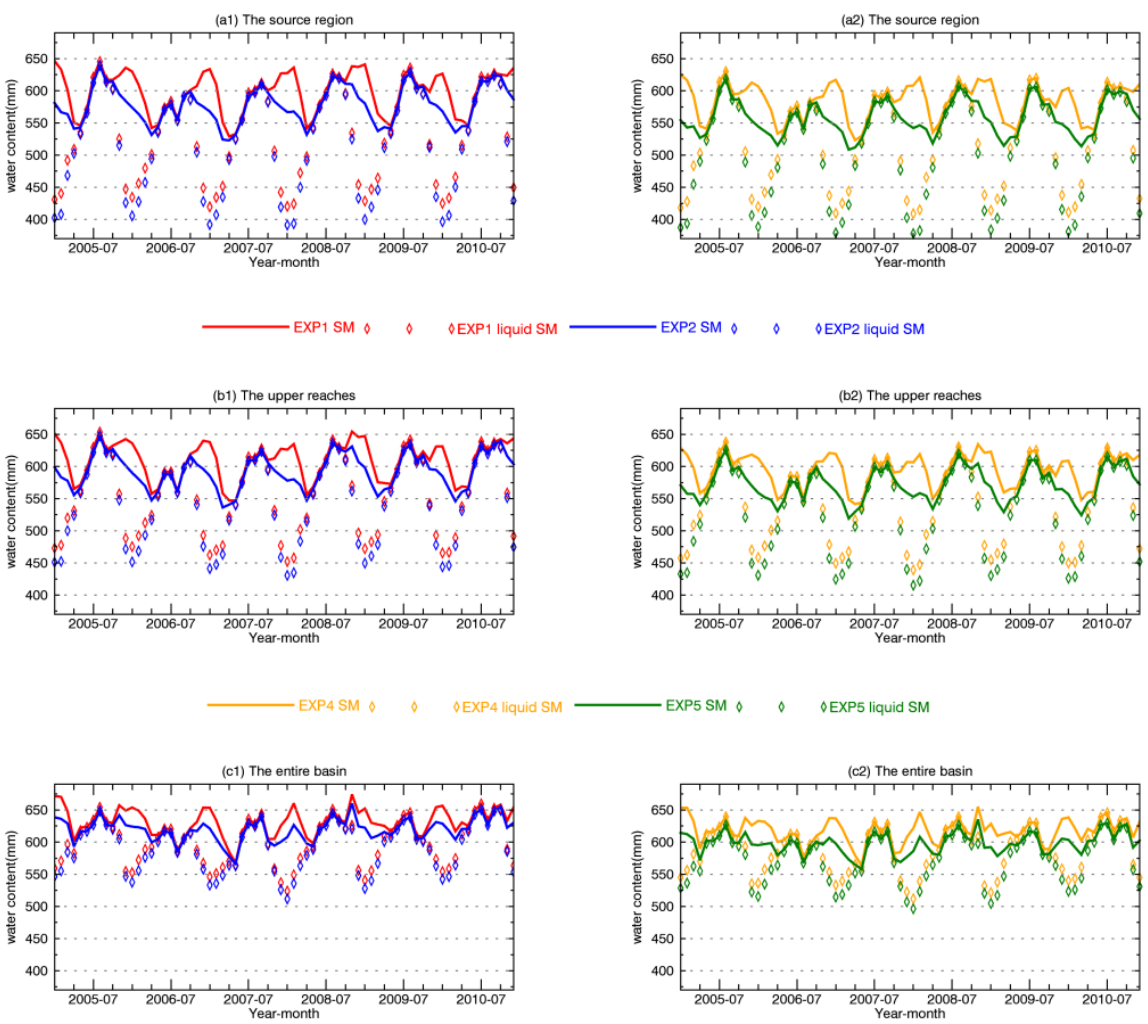

Figure 5.2. EXP1, EXP2, EXP4, and EXP5 produced monthly total soil moisture content (SM) and liquid SM, during the period 2005-2010, for the source region, upper reaches, and the entire Yangtze River basin, respectively.

EXP2 and EXP5 improve the runoff simulation through consistently producing more monthly runoff than EXP1 and EXP4, respectively. EXP2 and EXP5 favor the runoff simulation, by simply replacing Koren 99 (Koren et al., 1999) of EXP1 and EXP4 with NY06 (Niu and Yang, 2006) for the supercooled liquid water and frozen soil permeability schemes (Table 5.1). The major difference between Koren99 (Koren et al., 1999) and NY06 (Niu and Yang, 2006) for supercool liquid water in frozen soil $\left(\theta_{\text {liq }}\right.$ max,i) is the form of freezing-point depression equation (shown in equation (A1) and (A7)). Because of the extra term $\left(1+8 \theta_{i c e}\right)^{2}$, Koren99 tends to produce more liquid water than NY06, and has the increased interface between soil 
particles and liquid water due to the increase of ice crystals. This can explain why EXP1 and EXP4 has much more liquid water than EXP2 and EXP5, respectively, especially in winter, as shown in Figure 5.2. Furthermore, in terms of frozen soil permeability, NY06 uses the total SM (liquid soil water and soil ice content) to calculate the soil properties (soil matric potential and hydraulic conductivity), whereas they are defined by a function of liquid water volume in Koren99. When there is no or very little liquid water, the soil becomes too impervious that most of the snowmelt water flows laterally as surface runoff and little water infiltrates in to the soil, resulting in too high surface water and too low subsurface flow. Moreover, soil ice in NY06 has a smaller (nonlinear) effect on frozen fraction, resulting in more permeable soil than Koren99 does, because the latter assumes that soil ice has a linear effect on infiltration, leading to less infiltration. Therefore, It is not surprising that, in winter, EXP2 and EXP5 produce lower surface flow, but higher subsurface flow (not shown here), and higher total runoff (Figure 5.1) than EXP1 and EXP4, respectively. In summer, soil ice content is near zero and has almost negligible effect on $\theta_{\text {liq max,i }}$ and soil properties calculation, higher runoff produced by EXP2 and EXP5, thus, can be mainly attributed to the decreased ET due to the decreased soil moisture, as shown in Figure 5.3.

We compared EXP5 modeled monthly runoff against EXP2 and measured runoff, to investigate how different runoff schemes affect runoff simulation in the Yangtze River basin. As shown in Figure 5.1, EXP5 dramatically increase the amount of runoff in winter, which is much closer to the runoff observation, and hence a smaller MBE than EXP2 (Table 5.2), especially in the source region. However, Figure 5.1 and 5.4 show clearly that EXP5 produced runoff performs worse in amplitude and timing than EXP2 does. In EXP5, a simple groundwater model with a TOPMODEL-based runoff scheme is used as its runoff scheme, which has a dynamic coupling between the bottom soil layer and an unconfined aquifer. The groundwater recharge and discharge, driven by gravity or capillary forces, serves as the lower boundary condition of soil moisture (Niu et al., 2007). 

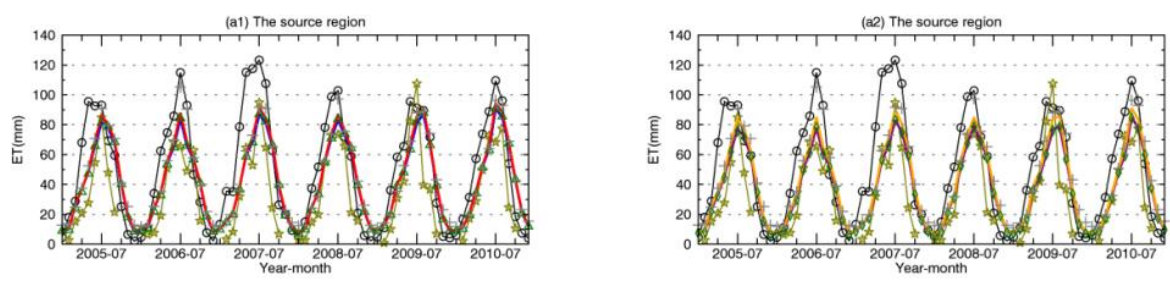

O O OSEBS * * * *GRACE-based
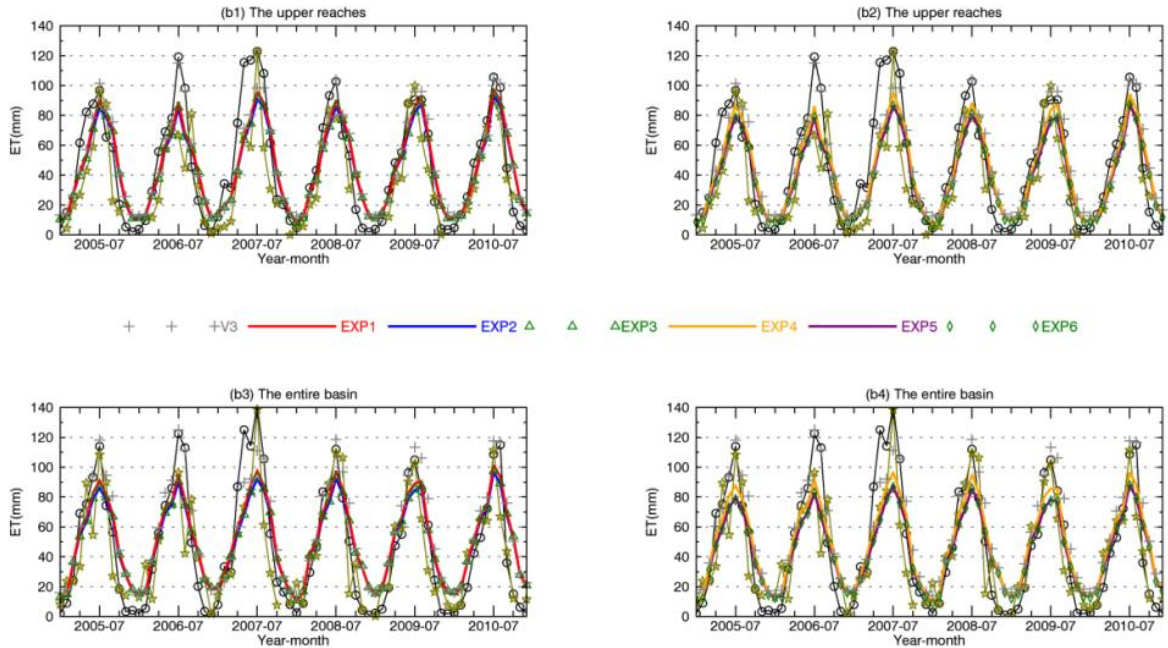

Figure 5.3. Observed, Noah V3 simulated, and EXP1-EXP6 produced monthly ET during the period 2005-2010 for the source region, upper reaches, and the entire Yangtze River basin, respectively. 

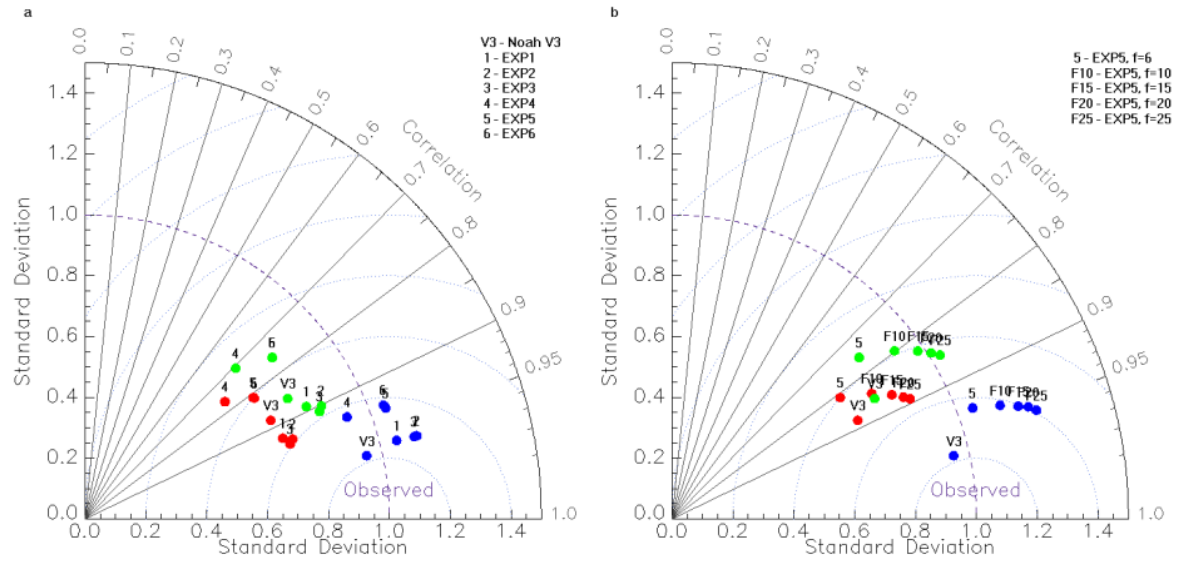

Figure 5.4. Taylor diagram (normalized) of (a) the observed, Noah V3 simulated, and EXP1-EXP6, (b) EXP5 with different decay factor $\mathrm{f}$ produced monthly runoff, during the period 2005-2010, for the source region (red), upper reaches (green), and the entire Yangtze River basin (blue).

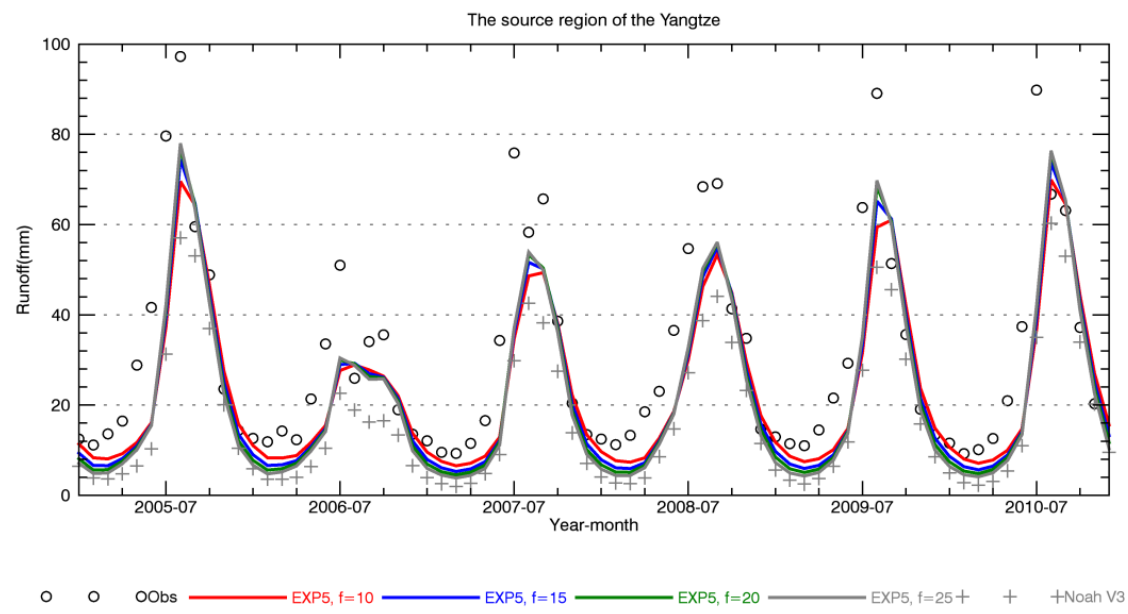

Figure 5.5. Observed, Noah V3, and EXP5 with different decay factor $f$ produced monthly runoff for the source region of the Yangtze during the period 2005-2010.

As shown in equation (B1-B3), runoff is exponentially dependent on the water table depth, and the decay factor, $f$, controls the shape and timing of the hydrograph recession curve and hence the seasonality of runoff. Because the decay 
factor $f$ affects the groundwater discharge and recharge, which gets smaller when $f$ is greater, thus the coupling strength between the soil and the unconfined aquifer gets smaller. Figure 5.5 shows the time series of runoff produced by EXP5 with different value of the decay factor $f$. As $f$ increases from the default value 6 to 25 , the timing and magnitude of the runoff produced by EXP5 gets closer to that of EXP2, which uses free drainage as the lower boundary condition of soil hydrology. Figure 5.4b also depicts that, as $f$ increases, EXP5 produced runoff has increased amplitude. This indicates that the groundwater dynamic plays an important role in runoff prediction and the lower boundary condition of soil moisture is the major contributor to runoff simulation. The problem of using free drainage at the model bottom as the lower boundary layer of soil moisture (EXP2) is that immediate removal of the drained water does not realistically represent the interactions of soil moisture, drained water and its underlying soil or aquifer, as the drained water should accumulate in its underlying soil or aquifer during wet seasons when recharge rates exceeds discharge rates, and be able to be drawn back to the soil column during dry seasons (Niu et al., 2011). In EXP5, the accumulated water in aquifer is allowed to, driven by capillary forces, draw back to the soil column in dry seasons, so more water is available for subsurface runoff generation, which can explain why EXP5 produced runoff is much higher in winter than EXP2. However, EXP5 produced runoff occurs later, and its peaks are smoother than EXP2 produced and observed runoff, especially in the source region, which is also mainly attributed to the groundwater buffering effects. After comparing the NSE of EXP5 with different value of the decay factor $f$ against observation, as shown in Table 5.2, we choose the value 15 (hereafter EXP5*) with which NSE reaches optimum in the source region, upper reaches and the entire basin.

We compared EXP2 and EXP5 produced runoff against EXP3, EXP6 modeled and observed, respectively, to investigate the effects of two $C_{h}$ schemes in the Noah-MP LSM on runoff simulation in the study area. EXP2 and EXP5, which uses Chen97's $C_{h}$ scheme, produces almost the same runoff as EXP3 and EXP6, which uses M-O $C_{h}$ scheme, respectively. Results show that Chen 97 and M-O $C_{h}$ schemes do not bring much difference on runoff estimates in the study area. However, previous research (Yang et al., 2011) pointed out $C_{h}$ scheme plays an important role not only in controlling skin temperature simulation, but also in runoff prediction. Because $C_{h}$ is 
an important parameter in potential ET calculation, runoff is also influenced to balance the water budget. Therefore, we cannot neglect the potential impacts of $C_{h}$ on runoff simulation, although small difference is shown here.

Table 5.2. MBE and NSE between observed and model estimated runoff for the period 2005-2010

\begin{tabular}{|c|c|c|c|c|c|c|}
\hline & \multicolumn{2}{|c|}{ The source region } & \multicolumn{2}{|c|}{ The upper reaches } & \multicolumn{2}{|c|}{ The entire basin } \\
\hline & $\begin{array}{c}\mathrm{MBE}(\mathrm{mm} / \\
\text { month) }\end{array}$ & NSE & $\begin{array}{c}\mathrm{MBE}(\mathrm{mm} / \mathrm{m} \\
\text { onth) }\end{array}$ & NSE & $\begin{array}{c}\mathrm{MBE}(\mathrm{mm} / \mathrm{m} \\
\text { onth) }\end{array}$ & NSE \\
\hline Noah & -14.41 & 0.35 & -11.83 & 0.39 & -10.22 & 0.64 \\
\hline EXP1 & -12.03 & 0.53 & -9.06 & 0.59 & -4.44 & 0.87 \\
\hline EXP2 & -10.81 & 0.61 & -7.58 & 0.67 & -2.52 & 0.90 \\
\hline EXP3 & -10.65 & 0.62 & -7.57 & 0.68 & -3.12 & 0.89 \\
\hline EXP4 & -10.08 & 0.37 & -6.99 & 0.38 & -2.26 & 0.85 \\
\hline EXP5 & -7.34 & 0.54 & -3.69 & 0.54 & -1.95 & 0.86 \\
\hline EXP6 & -7.22 & 0.55 & -3.68 & 0.54 & 1.46 & 0.85 \\
\hline EXP5, $\mathrm{f}=10$ & -7.94 & 0.59 & -4.37 & 0.58 & 0.79 & 0.85 \\
\hline EXP5, $\mathrm{f}=15$ & -8.53 & 0.62 & -4.93 & 0.60 & 0.28 & 0.84 \\
\hline EXP5, $\mathrm{f}=20$ & -8.94 & 0.63 & -5.31 & 0.61 & -0.07 & 0.83 \\
\hline EXP5, $f=25$ & -9.45 & 0.63 & -5.65 & 0.62 & 0.23 & 0.83 \\
\hline
\end{tabular}

We compared EXP2 and EXP5 produced runoff against EXP3, EXP6 modeled and observed, respectively, to investigate the effects of two $C_{h}$ schemes in the Noah-MP LSM on runoff simulation in the study area. EXP2 and EXP5, which uses Chen97's $C_{h}$ scheme, produces almost the same runoff as EXP3 and EXP6, which uses M-O $C_{h}$ scheme, respectively. Results show that Chen 97 and M-O $C_{h}$ schemes do not bring much difference on runoff estimates in the study area. However, previous research (Yang et al., 2011) pointed out $C_{h}$ scheme plays an important role not only in controlling skin temperature simulation, but also in runoff prediction. Because $C_{h}$ is an important parameter in potential ET calculation, runoff is also influenced to balance the water budget. Therefore, we cannot neglect the potential impacts of $C_{h}$ on runoff simulation, although small difference is shown here.

As shown in Figure 5.4, the modeled runoff has the closest agreement for the entire basin, but poorest matches in the source region, indicating that the source region is 
the most challenging part of the Yangtze River basin for land surface modeling. This is not surprising, because the source region is arid/semi-arid and high-altitude region, where land surface modelling still confronts difficulties and remains poorly performed. As shown in Table 5.2, EXP4, EXP5, and EXP6 produced runoff has remarkably smaller MBE than the Noah LSM, EXP1, EXP2 and EXP3 do, which indicates that the experiments using SIMGM runoff scheme perform better in the monthly runoff amount, especially in winter, than the ones with Schaake96 runoff scheme. However, the Noah LSM, EXP1, EXP2 and EXP3 generally perform better than EXP4, EXP5 and EXP6 in the Taylor diagram, which indicates that model runs with Schaake96 runoff scheme perform better than the ones with SIMGM runoff scheme in terms of timing and magnitude. This can be attributed to the suboptimum setting of the calibration parameter, the decay factor $f$, which controls the shape of timing of the hydrograph recession curve. We can also see that EXP2 and EXP3, EXP5 and EXP6 perform slightly better than EXP1and EXP4, respectively, which demonstrate that supercooled liquid water and frozen soil scheme of NY06 is superior to Koren99 for monthly runoff modelling in the Yangtze River basin.

\subsubsection{ET and soil moisture}

We computed the statistics of the monthly ET, during the period 2005-2010, for all the designed numerical experiments in the source region, the upper reaches, and the entire basin, respectively, as shown in Figure 5.6. The correlations of monthly ET produced by the numerical experiments with SEBS ET, and GRACE-based ET are quite good, which are all above 0.8 . These experiments produced ET generally has a better agreement with GRACE-based ET than SEBS ET, which has larger magnitude than others do, especially in the source region. 

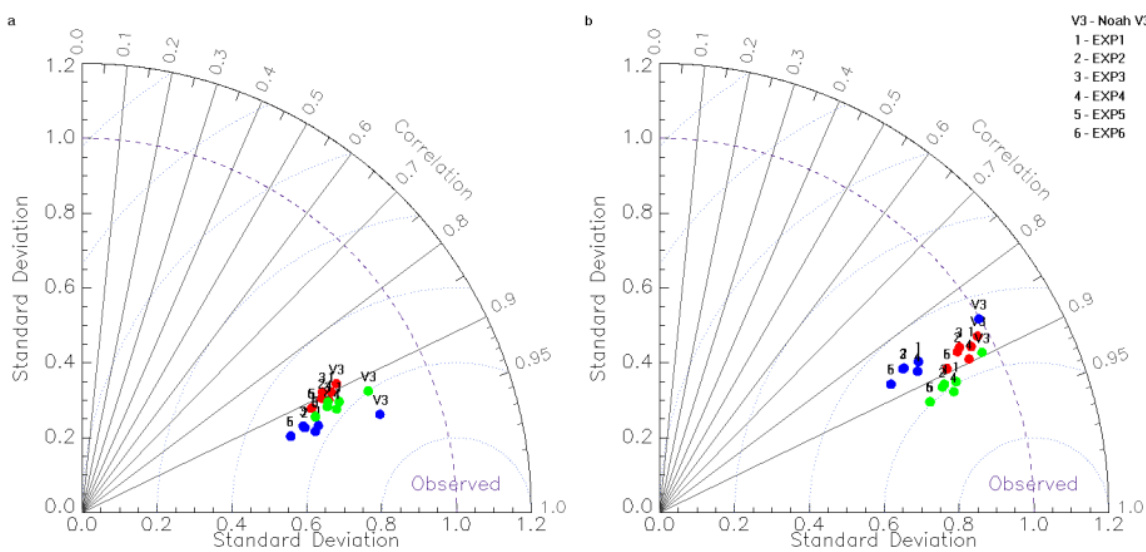

Figure 5.6. Taylor diagram (normalized) of (a) SEBS based, Noah V3 simulated, and EXP1-EXP6 produced monthly ET, and (b) GRACE based, Noah V3 simulated, and EXP1-EXP6 produced monthly

ET during the period 2005-2010 for the source region (red), upper reaches (green), and the entire Yangtze River basin (blue).

EXP1 and EXP4 produced ET has slightly closer agreements with SEBS and GRACE-based ET than EXP2 and EXP5, respectively, indicating that supercooled liquid water and frozen soil scheme of Koren99 performs slightly better than that of NY06 in the seasonality and magnitude of ET prediction. This is mainly due to the difference in summer, when NY06 produces a lower ET than Koren99 (Figure 5.6). As mentioned previously, Koren 99 produces more liquid water than NY06 does, which is shown in Figure 5.2. It seems that Koren99 produces too much ice in winter, which makes the total SM even higher than that in summer. High soil ice fraction in Koren99 leads to high frozen fraction, which has a greater effect on frozen fraction than NY06, resulting in too low soil permeability. In Koren99, the soil keeps too much water in winter, thus the runoff becomes low. In summer, when soil ice is near zero, the frozen soil has negligible effect on runoff, thus the higher ET produced by Koren99 in summer can be attributed to its wetter soil than NY06.

As also shown in Figures 5.2 and 5.3, ET produced by the experiments using the Noah-MP LSM with SIMGM, which has a smaller magnitude, fits the SEBS ET and GRACE-based ET slightly worse than the ones with free drainage, which is mainly 
due to the different ET in summer. This indicates that the lower boundary condition of soil moisture has a greater effect on ET in summer than winter, which can be attributed to the fact that, in summer, ET is larger and more easily affected by soil moisture condition. However, the decay factor $f$ seems have negligible effects on monthly ET at regional scale (not shown here). Moreover, the Noah LSM produced ET shows slightly better agreement than the ones produced by the Noah-MP LSM (EXP1-EXP6), which is due to the larger magnitude of the Noah LSM produced ET than others, as shown in Figure 5.3. However, Noah-MP (EXP1EXP6) significantly improves ET simulation in winter, when the Noah LSM produces too high ET, especially in the source region, and hence too low runoff.

\subsubsection{Comparison with GLDAS-Noah}

Figure 5.7 and Table 5.3 depict the comparisons of monthly observed, EXP5*, GLDAS-Noah, and the Noah LSM produced runoff in the source region, the upper reaches, and the entire basin, respectively. GLDAS-Noah produced runoff has large negative MBE, especially in the source region, and hence its model efficiency is poor. Although the Noah LSM also has large negative MBE, it performs better than GLDAS-Noah in terms of $\mathrm{R}^{2}$ and NSE. The Noah LSM simulated runoff has $250 \%, 30 \%$ and $36 \%$ improvement, in terms of model efficiency (NSE), on the GLDAS-Noah produced runoff in the source region, the upper reaches, and the entire Yangtze River basin, respectively. This improvement can be attributed to the more accurate forcing data, because this data benefits from merging information of 740 CMA operational stations, which is also confirmed by Chen et al. (2011). EXP5* remarkably reduces the MBE, and increases the model efficiency (NSE), which has $77 \%, 54 \%$, and $43 \%$ improvement on the Noah LSM produced runoff in the source region, the upper reaches, and the entire basin, respectively, and this improvement occurs both in summer and winter. This indicates that the hydrological simulation of the Yangtze River basin benefits quite a lot from the improved physics of NoahMP from the Noah LSM. The simulated runoff higher than observation is more reasonable, because the Yangtze River basin, especially the middle and lower reaches, involves intense water consumption such as irrigation, hydropower generation, and water supply, which makes the discharge from the gauging 
stations smaller than its actual value. Furthermore, the 'imperfect' atmosphere forcing may also be a cause for the uncertainties of the modelling results.
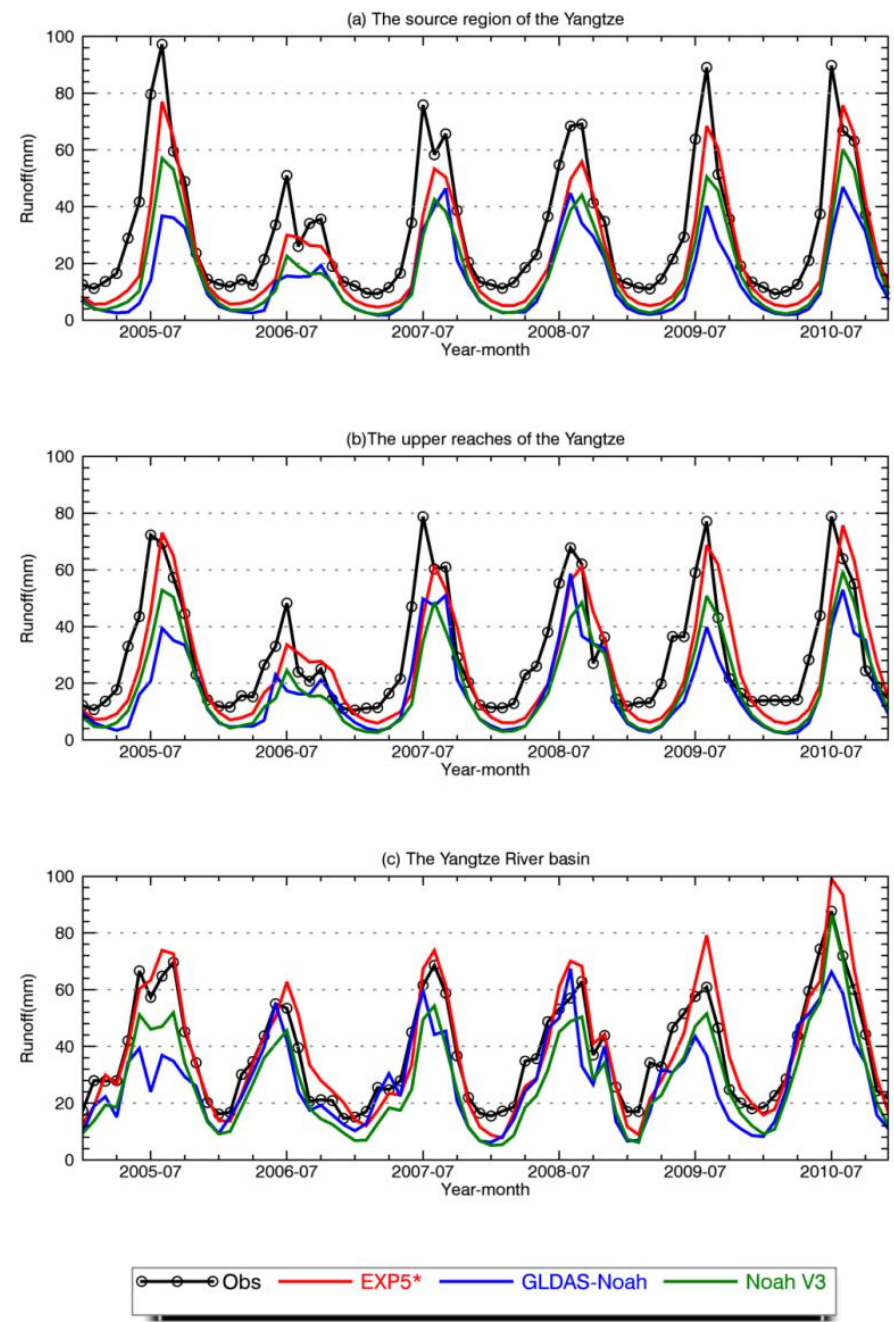

Figure 5.7. Observed, Noah V3, EXP5*(EXP5 with the decay factor $f$ of 15$)$, and GLDAS-Noah V2 produced monthly runoff, during the period 2005-2010, for the source region, upper reaches, and the entire Yangtze River basin, respectively. 


\subsection{Summary}

We conduct one numerical experiment with the Noah LSM and six experiments with different combinations of optional schemes of Noah-MP to investigate the major processes that control hydrology (runoff, ET, and soil moisture) in the Yangtze River basin. Results show that proper representation of the supercooled liquid water and frozen soil processes are important for modelling hydrological variables, especially for the arid and semi-arid region such as the source region of Yangtze. Furthermore, the lower boundary layer of soil moisture plays an important role in runoff generation and hence ET and soil moisture simulation. It can dramatically increase the monthly runoff amount in winter, which agrees well with the observation, although its magnitude and timing gets slightly worse. Moreover, although $C_{h}$ of $\mathrm{M}-\mathrm{O}$ and Chen97 do not seem to bring much difference in this study, its role on controlling hydrological modelling should not be neglected.

Table 5.3. MBE, R², RMSE, and NSE between observed and model estimated runoff for the period 20052010

\begin{tabular}{cccccc}
\hline $\begin{array}{c}\text { Model } \\
\text { estimates }\end{array}$ & Region & MBE(mm/month) & $\mathrm{R}^{2}$ & RMSE(mm/month) & NSE \\
\hline EXP5 & The & -8.53 & 0.76 & 20.93 & 0.62 \\
Noah V3 & source & -14.41 & 0.78 & 21.46 & 0.35 \\
GLDAS-Noah & region & -17.21 & 0.74 & 21.72 & 0.10 \\
EXP5 & The & -4.93 & 0.68 & 20.40 & 0.60 \\
Noah V3 & upper & -11.83 & 0.74 & 19.63 & 0.39 \\
GLDAS-Noah & reaches & -12.86 & 0.72 & 16.94 & 0.30 \\
EXP5 & The & 0.28 & 0.90 & 21.92 & 0.84 \\
Noah V3 & entire & -10.22 & 0.95 & 20.17 & 0.64 \\
GLDAS-Noah & basin & -10.16 & 0.78 & 13.28 & 0.47 \\
\hline
\end{tabular}

We calibrated the decay factor $f$ of SIMGM in EXP5 to adjust the coupling strength between soil columns and their underlying aquifer. The calibrated Noah-MP experiment (EXP5*) further improves the hydrological modelling in the study area. 
We compared the runoff produced by the Noah LSM, EXP5*, and GLDAS-Noah against observation. The results show that the improvement of EXP5* compared with GLDAS-Noah production, in terms of water fluxes and states, comes from the more accurate atmosphere forcing data and the enhanced physics of the Noah-MP LSM. 


\section{Appendix A: Options for supercooled liquid water and frozen soil scheme in Table 5.1}

\section{NY06}

When soil water freezes, water close to soil particles remains in liquid form due to the absorptive and capillary forces exerted by soil particles. Thus, only the excessive liquid water beyond $\theta_{\text {liqmax }, I}$, the upper limit of the supercooled liquid water, can get frozen. $\theta_{\text {liqmax }, I}$ is derived from the following form of freezing-point depression equation:

$$
\Psi_{\text {sat }}\left(\frac{\theta_{\text {liqmax }}}{\theta_{\text {sat }}}\right)^{-b}=\frac{10^{3} L_{f}\left(T-T_{f r z}\right)}{g T},
$$

where $\theta_{\text {liqmax }}$ is the maximum liquid water when the soil temperature is below the freezing point; $T$ and $T_{f r z}$ are soil temperature and freezing point $(\mathrm{K})$, respectively (Fuchs et al., 1978); $L_{f}$ is the latent heat of fusion $\left(\mathrm{J} \mathrm{kg}^{-1}\right) ; \mathrm{g}$ is the gravitational acceleration ( $\left.\mathrm{m} \mathrm{s}^{-2}\right) ; \theta_{\text {sat }}$ is the saturated soil water content (porosity); and $\psi_{\text {sat }}$ is the saturated soil matric potential depending on the soil texture, and $b$ is the Clapp-Hornberger parameter.

Additional water may be ice depending on the available energy. The liquid water content, thus, for the next time step $(\mathrm{N}+1)$ follows:

$$
\theta_{\text {liq }}^{N+1}=\min \left(\theta_{\text {liqmax }}, \theta^{N}\right)
$$

where $\theta^{N}$ is the total volumetric soil moisture at time step $\mathrm{N}$, including liquid water content and ice content. The soil ice content $\left(\theta_{\text {ice }}\right)$ is computed from the following equation:

$$
\theta_{i c e}^{N+1}=\min \left(\theta^{N}-\theta_{\text {liq }}^{N+1}, \theta_{i c e}^{N}+R_{f m} \Delta t\right)
$$

An area of a given GCM grid cell can be separated to permeable and impermeable areas. It assumes that the effects of these impermeable and permeable areas on infiltration can be linearly aggregated, and both the fractional permeable and 
impermeable areas share the same total soil moisture of the grid cell. With these assumptions, the water flux within the soil of a GCM can be expressed as:

$$
q=\left(1-F_{f r z}\right) q_{u}+F_{f r z} q_{f r z},
$$

where $F_{f r z}$ is the fractional impermeable (frozen) area, and $q_{u}$ and $q_{f r z}$ are water flux in the unfrozen and frozen areas, respectively. Assuming $q_{f r z}=0$, the above equation can become:

$$
q=\left(1-F_{f r z}\right) q_{u}=-\left(1-F_{f r z}\right) k_{u} \frac{\partial\left(\psi_{u}+z\right)}{\partial z},
$$

where $\theta=\theta_{\text {ice }}+\theta_{\text {liq }}$ is the total grid-cell volumetric soil moisture.

The fractional impermeable area is parameterized as a function of soil ice content at a layer:

$$
F_{f r z}=e^{-\alpha\left(1-\theta_{i c e} / \theta_{s a t}\right)}-e^{-\alpha},
$$

where $\alpha=3.0$ is an adjustable scale-dependent parameter.

\section{Koren99}

It proposed an alternative method for representing the maximum supercooled soil water by iteratively solving the following equation, a variant of the freezing-point depression equation:

$$
\left(1+8 \theta_{\text {ice }}\right)^{2} \psi_{\text {sat }}\left(\frac{\theta_{\text {liqmax }}}{\theta_{\text {sat }}}\right)^{-b}=\frac{10^{3} L_{f}\left(T-T_{f r z}\right)}{g T},
$$

where $\theta_{\text {liqmax }}$ is the maximum liquid water when the soil temperature is below the freezing point; $T$ and $T_{f r z}$ are soil temperature and freezing point (K), respectively (Fuchs et al., 1978); $L_{f}$ is the latent heat of fusion ( $\left.\mathrm{J} \mathrm{kg}^{-1}\right)$; $\mathrm{g}$ is the gravitational acceleration ( $\left.\mathrm{m} \mathrm{s}^{-2}\right) ; \theta_{\text {sat }}$ is the saturated soil water content (porosity); $\theta_{\text {ice }}$ is the soil ice content; and $\psi_{\text {sat }}$ is the saturated soil matric potential depending on the soil texture, and $\mathrm{b}$ is the Clapp-Hornberger parameter. 


\section{Appendix B: Options for runoff and groundwater in Table 5.1}

\section{SIMGM}

It uses a simple TOPMODEL-based runoff model (Niu et al., 2005) to compute surface runoff and base flow (groundwater discharge), which are both parameterizes as exponential functions of the water table depth. Below the bottom of the Noah soil column, an unconfined aquifer is used to account for the exchange of water between the soil and the aquifer. Surface runoff is parameterized as:

$$
R_{s f}=F_{\text {sat }} p+\left(1-F_{\text {sat }}\right) \max (0,(\mathrm{p}-\mathrm{I}))
$$

where $\mathrm{p}$ is the effective precipitation intensity $\left(\mathrm{kg} \mathrm{m}^{-2} \mathrm{~s}^{-1}\right)$, I is maximum soil infiltration capacity $\left(\mathrm{kg} \mathrm{m}^{-2} \mathrm{~s}^{-1}\right)$, which is dependent on soil properties and moisture, and $F_{\text {sat }}$ is the fraction of saturated area and is parameterized as:

$$
F_{\text {sat }}=\left(1-F_{f r z}\right) F_{m a x} e^{-0.5 f\left(z_{\nabla}-z_{b o t}^{\prime}\right)}+F_{f r z}
$$

where $F_{f r z}$ is a fractional impermeable area as a function of the soil ice content of the surface soil layer, $z_{\nabla}$ is the water table depth $(\mathrm{m}), z_{b o t}^{\prime}$ is the depth of the model bottom, which is $2 \mathrm{~m}$, and $F_{\max }$ is the potential or maximum saturated fraction for a grid cell and can be derived from high-resolution sub grid topography of a model grid cell using the TOPMODEL concepts. The default value of $F_{\max }$ is 0.38 , which is derived from the HYDRO1K $1 \mathrm{~km}$ topographic index (or wetness index, WI) data.

Subsurface runoff $\left(R_{s b}\right)$ is parameterized as

$$
R_{s b}=R_{s b, \max } e^{-\Lambda-f\left(z_{\nabla}-z_{b o t}^{\prime}\right)}
$$

where $R_{s b \text {,max }}$ is the maximum subsurface runoff when the grid cell mean water table depth is zero, which is set $5.0 \times 10^{-4} \mathrm{~m} \mathrm{~s}^{-1}$ globally due to the calibration against global runoff data through sensitivity tests (Niu et al., 2007). $\Lambda$ is the grid cell mean WI, the global mean of which is 10.46 derived from HYDRO1K $1 \mathrm{~km}$ WI data.

The temporal variation of the water stored in the unconfined aquifer, $W_{a}(\mathrm{~mm})$, is parameterized as 


$$
\frac{d W_{a}}{d t}=Q-R_{s b}
$$

where $Q$ is the recharge rate $\left(\mathrm{mm} \mathrm{s}^{-1}\right)$, which is positive when water enters the aquifer. It is parameterized as

$$
Q=-K_{b o t} \frac{-z_{\nabla}-\left(f_{m i c} \psi_{b o t}-z_{b o t}\right)}{z_{\nabla}-z_{b o t}},
$$

where $K_{b o t}$ is hydraulic conductivity of the bottom soil layer $\left(\mathrm{mm} \mathrm{s}^{-1}\right)$. The $f_{\text {mic }}(0.2$ in this study) is the fraction of micropore content in the bottom layer soil, which is introduced to limit the upward flow (depending on the level of structural soil) and ranges from 0.0 to 1.0. When $f_{\text {mic }}=0.0$ (structural soil or aquifers without microspores), equation (B5) is reduced to free drainage $\left(Q=K_{b o t}\right)$. When $f_{\text {mic }}=1.0$ (textural soil full of micropores), equation (B5) represents a maximum effect of groundwater on soil moisture (Niu et al., 2011). $\psi_{b o t}$ is the matric potential (mm), and $z_{b o t}(1.5 \mathrm{~m}$ in this study $)$ is the midpoint of the bottom soil layer.

\section{Schaake96}

Its surface infiltration scheme follows a simple water balance model (Schaake et al., 1996) for its treatment of the subgrid variability of precipitation and soil moisture. Surface water is generated when the rain intensity exceeds the infiltration capacity and is calculated as:

$$
\mathrm{R}_{\mathrm{sf}}=\mathrm{P}-\mathrm{I}_{\max },
$$

where $R_{s f}$ is the surface runoff $\left[\mathrm{m} \mathrm{s}^{-1}\right], P$ is the rain intensity $\left[\mathrm{m} \mathrm{s}^{-1}\right.$, and $I_{\max }$ is the maximum infiltration capacity $\left[\mathrm{m} \mathrm{s}^{-1}\right]$.

$I_{\max }$ can be written as:

$$
I_{\max }=P \frac{D_{b}[1-\exp (k d t)]}{P+D_{b}[1-\exp (k d t)]},
$$

where $D_{b}$ is the total soil moisture deficit in the soil column $\left[\mathrm{m}^{3} \mathrm{~m}^{-3}\right]$ and kdt is a constant [-] defined by,

$$
\mathrm{kdt}=\mathrm{kdt}_{\mathrm{ref}} \frac{\mathrm{K}_{\mathrm{s}}}{\mathrm{K}_{\mathrm{ref}}}
$$


where $\mathrm{K}_{\mathrm{s}}$ is the saturated hydraulic conductivity [m s-1, $\mathrm{kdt}_{\mathrm{ref}}$ and $\mathrm{K}_{\mathrm{ref}}$ are experimentally determined parameters set to $3.0[-]$ and $2.010^{-6}\left[\mathrm{~m} \mathrm{~s}^{-1}\right]$ for largescale simulations, respectively.

$$
R_{s b}=\operatorname{SLOPE} * \mathrm{~K}(\theta), \quad(\mathrm{B} 9)
$$

where $\mathrm{K}(\theta)$ is the hydraulic conductivity [ $\mathrm{m} \mathrm{s}^{-1}$, and SLOPE is the slope of the earth's surface [-]. 


\section{CHAPTER 6}

\section{Analysis of Long-term Terrestrial Water Storage Variations Caused by Climate Variability in the Yangtze River Basin}





\section{Chapter 6 Analysis of long-term terrestrial water storage variations caused by climate variability in the Yangtze River basin}

\section{This chapter is also based on:}

Huang, Y., M. S. Salama, M. S. Krol, R. van der Velde, A. Y. Hoekstra, Y. Zhou, and $\mathrm{Z}$. Su, 2013: Analysis of long-term terrestrial water storage variations in the Yangtze River basin. Hydrol. Earth Syst. Sci., 17, 1985-2000.

\subsection{Abstract}

This chapter analyzes the 32 years of spatial and temporal total water storage (TWS) variations caused by climate variability in the Yangtze River basin. It shows that climate-induced TWS in the Yangtze River basin has decreased significantly since the year 1998. The driest period in the basin occurred between 2005 and 2010, and particularly in the middle and lower Yangtze reaches. The TWS figures changed abruptly to persistently high negative anomalies in the middle and lower Yangtze reaches in 2004. The year 2006 is identified as major inflection point, at which the system starts exhibiting a persistent decrease in TWS. Comparing these TWS trends with independent precipitation datasets shows that the recent decrease in TWS can be attributed mainly to a decrease in the amount of precipitation. 


\subsection{Introduction}

As a key component of terrestrial and global hydrological cycles, TWS strongly influences water, energy, and biogeochemical fluxes, thereby playing a major role in the Earth's climate system (Famiglietti, 2004). TWS is not only an indicator of the Earth's climate variability, but also affects various components of the Earth's hydrological cycle (Niu and Yang, 2006). Soil moisture plays a key role in both the water and energy cycles through its impact on the energy partitioning at the surface, and soil moisture also has links with the biogeochemical cycle via plant transpiration and photosynthesis (Seneviratne et al., 2010). Snow cover has a strong influence on the onset of the summer monsoon and runoff production in spring (Ding et al., 2009). Therefore, the spatial and temporal variability in TWS due to climate change and human-induced impacts both form important components in the water and energy cycles, and should be taken into account in river basin management.

As described in Chapter 1, global Data assimilation products such as Interim Reanalysis Data (ERA-Interim) and Global Land Data Assimilation System (GLDAS) combine the virtues of in situ data, remotely sensed observations, and modeling. The models in these systems simulate the main components of TWS and, by fusing these components with other data sources, reduce uncertainties in the hydrological interpretations. These systems have been extensively applied in TWS and related studies, and have, for example, been utilized in regional, continental, and global TWS variation analysis (Chen et al., 2005; Seneviratne et al., 2004; Syed et al., 2008). The main components of TWS such as soil moisture (SM) and snow water equivalent (SWE) can also be simulated by land surface models (LSMs). This simulated TWS may have higher accuracy than public global data assimilation products, because of the improved atmospheric forcing data and more realistic representation of physical processes for the study area.

In Chapter 3, two public global data assimilation products, the ERA-Interim and GLDAS-Noah, have been evaluated, and the results show that apparently the ERAInterim datasets have higher accuracy and reliability for the Yangtze River basin. In Chapters 4 and 5, the Noah model simulation has achieved higher accuracy by 
the improved atmospheric forcing data and more realistic representation of physical processes for the study area. However, the ERA-Interim datasets still perform relatively better, as shown in Figures 3.1 and 3.3. Therefore, the ERAInterim data are selected to investigate the TWS variations mainly controlled by the climate variability.

In this study, we focus on the analysis of the long-term space-time variability in TWS of the Yangtze River basin, with the aim to improve our understanding of the water cycle and aid management of the water resources. The specific objectives of this chapter are (1) to use the ERA-Interim dataset to examine the climatology of the spatial pattern of TWS in the basin, and (2) to detect trends and abrupt changes, as well as their possible causes.

\subsection{Methods}

\subsubsection{Statistical analysis}

Trend analyses involve linear regression and the non-parametric Mann-Kendall (MK) test (Mann, 1945; Kendall, 1975). A linear regression model is used to compute the annual trend in the TWS for each pixel. The MK test is a rand-based procedure and is applied to detect the significance of the trends. The MK test statistics are given by

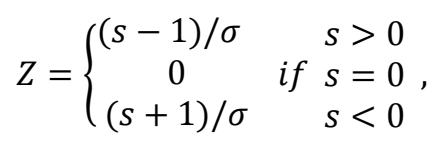

where

$$
s=\sum_{i=1}^{n-1} \sum_{j=i-1}^{n} \operatorname{Sgn}\left(X_{j}-X_{i}\right)
$$

and

$$
\operatorname{Sgn}\left(X_{j}-X_{i}\right)=\left\{\begin{array}{cr}
+1 & X_{j}-X_{i}>0 \\
0 & \text { if } X_{j}-X_{i}=0 \\
-1 & X_{j}-X_{i}<0
\end{array}\right.
$$


The $\sigma$ term is given by

$$
\sqrt{1 / 18\left(n(n-1)(n-5)-\sum_{t} t(t-1)(2 t+5)\right.}
$$

and $X_{j}$ and $X_{i}$ are the sequential data values, $n$ is the dataset record length, $t$ is the extent of any given tie (the number of annual maxima in a given tie), and $\Sigma$ is the summation of all ties. Positive and negative values of $Z$ indicate increasing and decreasing trends, respectively. The statistic $Z$ follows a normal distribution $N(0,1)$ (Burn and Hag Elnur, 2002; Yang et al., 2010). To analyze whether the trend is stationary in the TWS anomalies, the Mann-Kendall-Sneyers (MKS) test (Sneyers, 1975) is also applied. This test, a sequential version of the MK test, enables not only detection of significant trends, but also approximation of the transition point in the temporal behavior of a series. Let $x_{1}, \ldots, x_{n}$ be the data points. For each element $x_{i}$, the number $n_{i}$ of element $x_{j}$ proceeds it $(j<i)$ such that $x_{j}<x_{i}$ is computed. Under the null hypothesis (no trend), the test statistic $t_{k}=\sum_{i=1}^{k} n_{i}$ is normally distributed, with the mean and variance given by

$$
\begin{gathered}
\bar{t}_{k}=E\left(t_{k}\right)=\frac{k^{2}-k}{4}, \\
\bar{\sigma} t_{k}^{2}=\operatorname{var}\left(t_{k}\right)=\frac{k(k-1)(2 k+5)}{72},
\end{gathered}
$$

Let $U F_{k}=\left(t_{k}-\overline{t_{k}}\right) /\left(\bar{\sigma} t_{k}^{2}\right)^{0.5}$ be the normalized variable, which is the forward sequence. This principle can be usefully extended to the backward sequence $U B_{k}$, which is calculated using the same equation but with a reversed series of data. When points in the forward series are outside the confidence interval, this indicates the detection of a significantly increasing $(U F>0)$ or a significantly decreasing $(U F<0)$ trend. If an intersection occurs between $U F$ and $U B$ within the confidence interval, this indicates an inflection ( $\mathrm{Li}$ and Wang, 2004; Li et al., 2007; Moraes et al., 1998).

\subsubsection{Standardized anomalies}


Annual standardized anomalies are calculated by using the monthly values subtracted with the annual mean and divided by the annual standard deviation of the annual mean, which can be expressed as follows:

$$
A_{i j}=\frac{T W S_{i j}-\overline{T W S_{J}}}{\sigma_{j}},
$$

with

$$
\begin{gathered}
\overline{T W S_{J}}=1 / 12 \sum_{i=1}^{12} T W S_{i j} \\
\sigma_{j}=\left(1 / 12 \sum_{i=1}^{12}\left(T W S_{i j}-\overline{T W S_{J}}\right)^{2}\right)^{0.5}
\end{gathered}
$$

where $A_{i j}$ is the annual TWS standardized anomaly in the $i$ th month of the $j$ th year, $T W S_{i j}$ is the TWS in the $i$ th month of the $j$ th year, $\overline{T W S_{j}}$ is the mean TWS of the all months in the $j$ th year, and $\sigma_{j}$ is the standardized deviation of all months in the $j$ th year.

Monthly standardized anomalies of the TWS are calculated by the monthly TWS minus the corresponding monthly value of the annual cycle, and then divided by the standard deviation of the values of the same months within the period 1979 to 2010, in order to eliminate the influence of inter-annual variability for intra-annual analysis. Monthly standardized anomalies of the TWS can be expressed as follows:

$$
M_{i j}=\frac{T W S_{i j}-\overline{T W S_{l}}}{\sigma_{i}}
$$

with

$$
\begin{gathered}
T W S_{i}=1 / 32 \sum_{j=1979}^{2010} T W S_{i j} \\
\sigma_{i}=\left(1 / 32 \sum_{i=1979}^{2010}\left(T W S_{i j}-\overline{T W S_{l}}\right)^{2}\right)^{0.5}
\end{gathered}
$$


where $M_{i j}$ is the monthly TWS standardized anomaly in the $i$ th month of the $j$ th year. The subscripts $\mathrm{i}$ and $\mathrm{j}$ represent the $i$ th month and $j$ th year, respectively; $T W S_{i}$ is the TWS of the $i$ th month averaged over all the years; $\sigma_{i}$ is the standardized deviation of $i$ th month TWS over all the years.
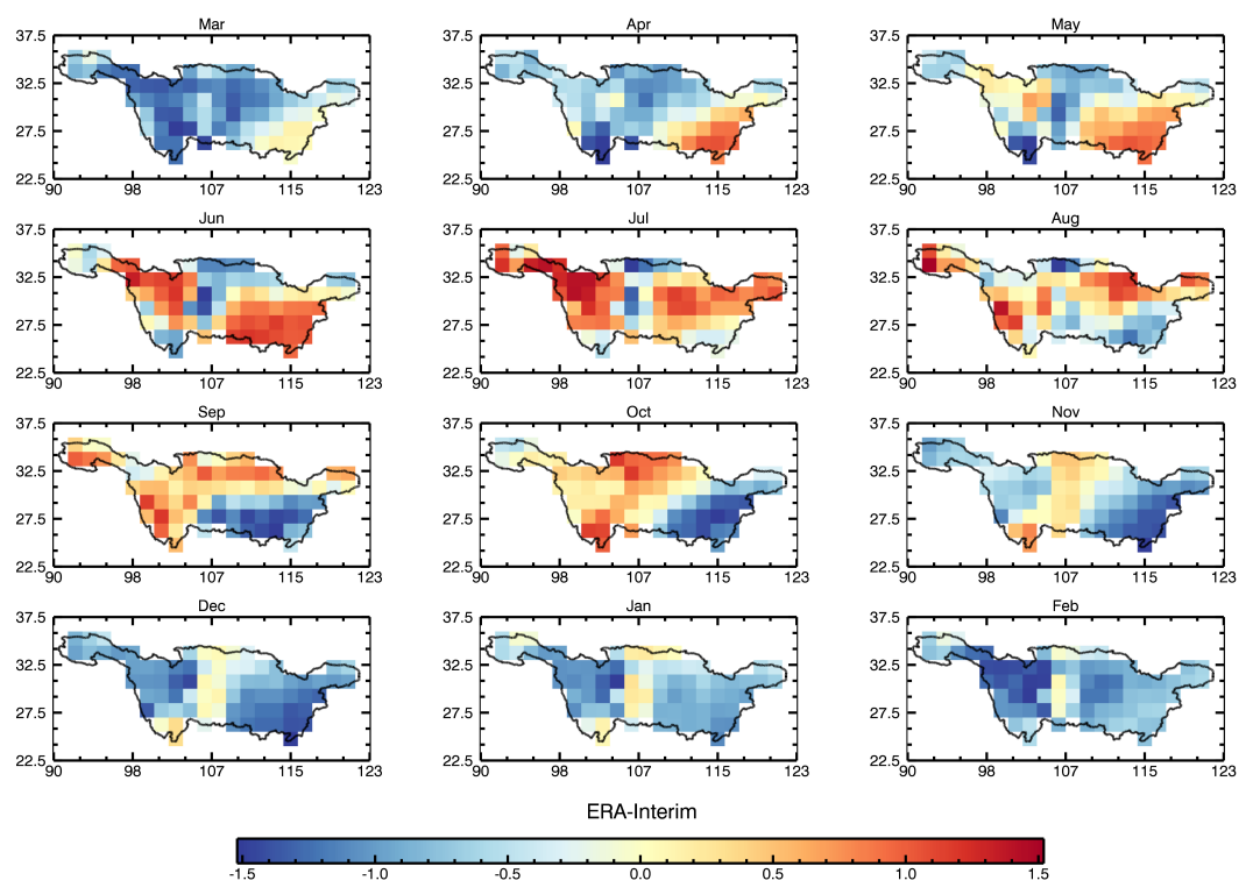

Figure 6.1.Spatial patterns of monthly averaged TWS annual standardized anomalies computed from ERA-Interim for the period January 1979 till December 2010.

\subsection{Results and discussion}

\subsubsection{Climatology}

The spatial distribution of TWS and terrestrial water storage change (TWSC) climatological annual standardized anomalies derived from ERA-Interim are shown in Figures 6.1 and 6.2. After suffering the dry season (December-February), the southeast corner of the Yangtze River basin starts to become wet during March to May, due mainly to the south China rainfall belt extension and the mean precipitation increase in the lower basin (Ding and Chan, 2005; Qian et al., 2002). 
High, positive TWS standardized anomalies emerge in most of the Yangtze basin during June to October (Figure 6.1), and show a large increase in July compared to June (Figure 6.2), corresponding with the intensive precipitation observed along the whole Yangtze River from mid-June to mid-July (Ding, 1992), called the Meiyu in China. According to the climatological rainfall differences between May and June and between June and July (Qian et al., 2002, Figure 4), the increased rainfall in June compared to May appears in the Plateau and in southwest China, with the center lying in the upper Yangtze reaches.
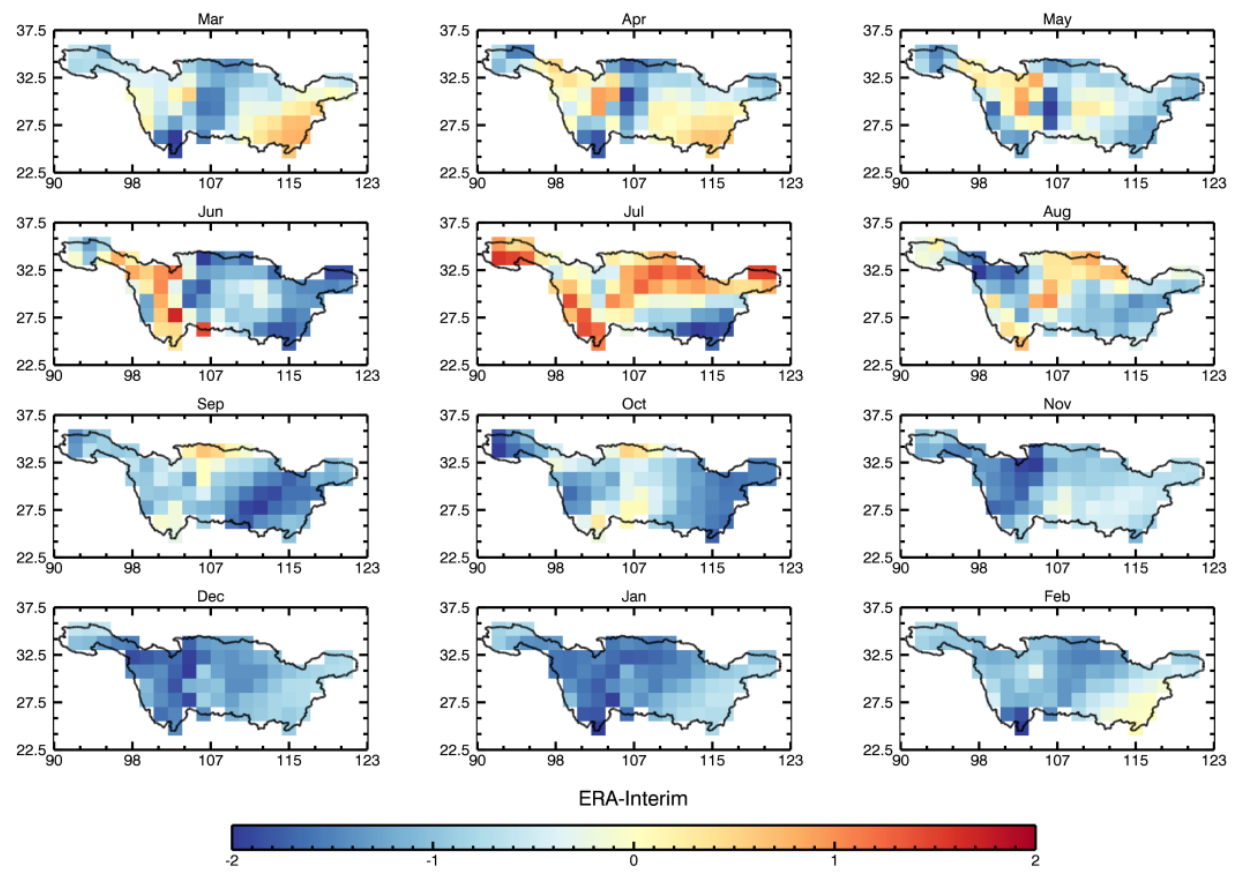

Figure 6.2.Spatial patterns of monthly averaged TWSC annual standardized anomalies computed from ERA-Interim for the period January 1979 till December 2010.

Another area of increased rainfall is located along the eastern coastland with its center in the lower Yangtze reaches. There is no obvious increase in rainfall in the central part of China. In July, the increased rainfall has migrated to the north of the lower Yangtze River basin, while rainfall is steadily increasing in the upper parts. This pattern of change in precipitation from May to July resembles the TWS pattern derived for ERA-Interim (Figure 6.1). After July, the TWS anomalies in the 
middle and lower Yangtze reaches decrease sharply, while they stay quite high and positive in the upper Yangtze reaches till October, mainly due to the continuous rainy season from mid-June to mid-September.
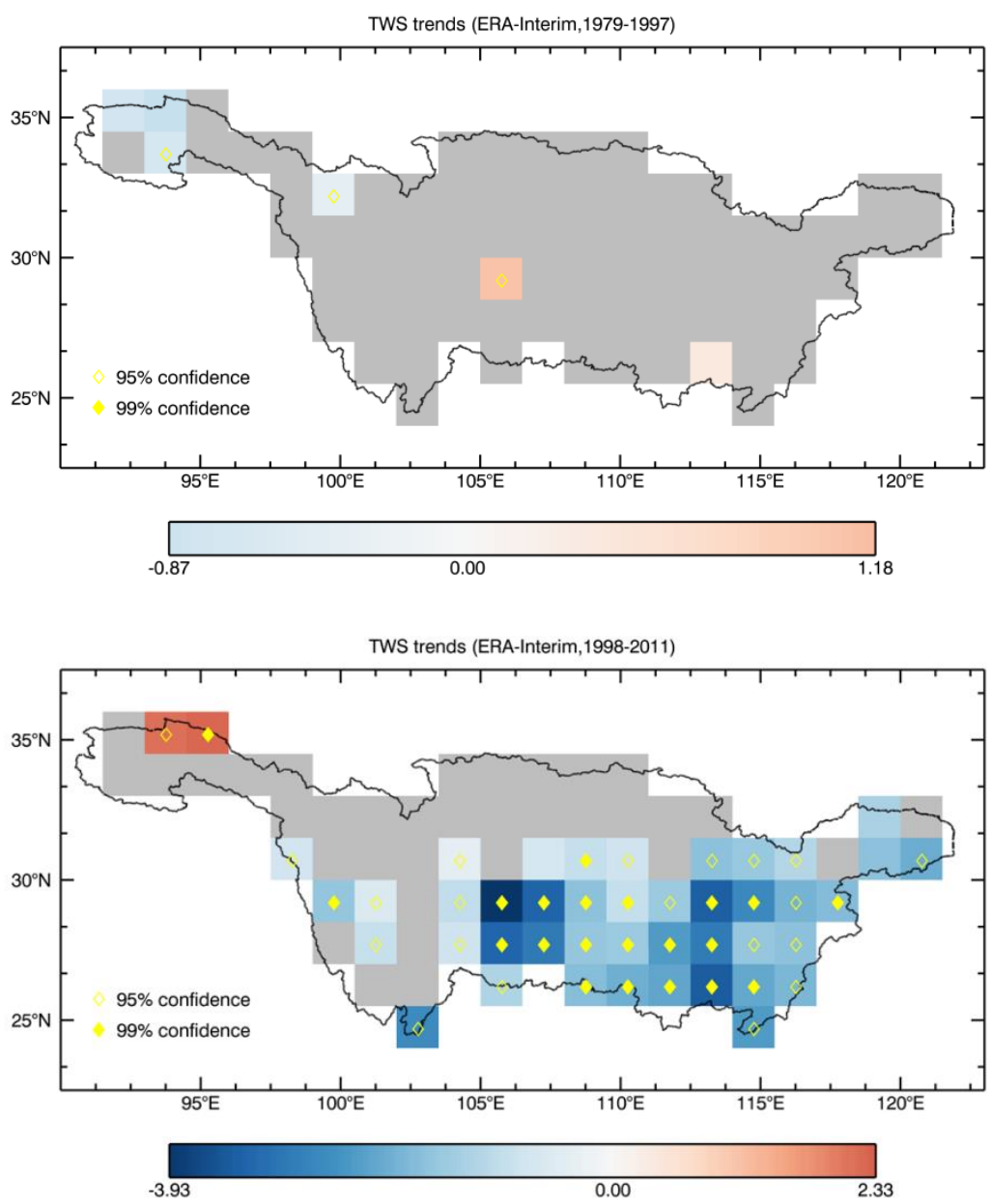

Figure 6.3.ERA-Interim estimated TWS annual trends between 1979 and 1997, and between 1998 and

2010, in millimeters per year (gray grids cells represent insignificant trends; cells with an empty diamond indicate the trend surpasses the $95 \%$ confidence level; cells with a filled diamond indicate the trend surpasses the $99 \%$ confidence level; others indicate the trend surpasses the $90 \%$ confidence level). 
This striking consistency between TWS and the rainfall pattern is not unexpected. That higher precipitation leads to higher soil moisture can generally be considered predictable, though there are a few exceptions. For instance, in the case of intense precipitation with rates beyond the infiltration rate, or precipitation over very wet or saturated areas, the rainfall anomalies will results in runoff anomalies rather than soil moisture (Dunne, 1978; Horton et al., 1933). Nevertheless, except in the extreme cases, there is an obvious and direct response of soil moisture to precipitation. On the other hand, the feedback, via the return path from soil moisture through evapotranspiration to precipitation, can also play an important role in the TWS variability, though a weaker one (Seneviratne et al., 2010). Abundant previous research (Dirmeyer, 2011; Jung et al., 2010; Wei et al., 2012) shows that the Yangtze River basin is dominated by wet soil moisture regimes, where soil moisture does not mainly control the variability in evapotranspiration and has only a minor impact on the change in rainfall. Dirmeyer (2011) also confirms that soil moisture neither typically provides feedback to the atmosphere nor has a damping effect on climate variability. Thus, it is reasonable to speculate that the TWS variability in the Yangtze River basin is mainly controlled by largescale atmospheric circulations, as is also established by Wei et al. (2012). Moreover, as displayed in Figure 6.1, the Yangtze River basin suffered the highest TWS anomalies during June-July, which implies a high flood risk during this period, since runoff is sensitive to soil moisture content under wet soil regimes. When soil moisture is very high and soil becomes saturated, high precipitation variability may lead to high runoff variability, which cannot be damped by soil moisture storage (Seneviratne et al., 2010).

It should be recognized that the TWS pattern in the upper Yangtze reaches is completely different from that in the middle and lower Yangtze reaches, which may be explained by large-scale circulation and heterogeneous land-surface conditions. The upper Yangtze reaches are mainly influenced by the South Asian (or Indian) summer monsoon, and the middle and lower Yangtze reaches are controlled by the East Asian summer monsoon (Ding and Chan, 2005). The seasonal process of the Asian summer monsoon plays a crucial role in heat and moisture transport and the hydrological cycle. Related rainfall systems perform differently in the upper reaches than in the middle and lower reaches (Qian et al., 
2002). Since the topography in the upper Yangtze reaches is totally different from that in the middle and lower reaches (Figure 2.1), land-surface heterogeneities in temperature are expected (Giorgi et al., 1997; Salama et al., 2012). The land cover and hydrological conditions differ in the two areas (Piao et al., 2010). The inhomogeneous surface results in heterogeneity in surface energy partitioning, which in turn has an impact on land-atmosphere interactions (Brunsell et al., 2011; Ma et al., 2008). Therefore, different responses are expected by land-surface systems in the upper Yangtze reaches than in the middle/lower reaches. For example, soil moisture exerts a significant positive control on the maximum and mean temperature in the middle/lower reaches during summer, while no significant control is elicited in the upper reaches. Furthermore, while soil moisture and precipitation are positively coupled in the upper Yangtze reaches, this coupling is negative for the middle and lower Yangtze reaches (Zhang et al., 2011).

\subsubsection{TWS trend analysis}

In Figure 6.3, the ERA-Interim dataset shows decreasing TWS trends over large parts of the Yangtze River basin between 1998 and 2010, which match the descending trend in the soil moisture column from microwave satellite observations between 1998 to 2008 (Jung et al., 2010). It shows significantly decreasing trends (most of which surpass the $95 \%$, while some even surpass the $99 \%$ confidence level) in the middle and lower reaches, with a maximum of $-3.93 \mathrm{~mm}$ $\mathrm{yr}^{-1}$. The upper reaches suffer milder decreases and even insignificant trends in some parts during the period 1998 to 2010. Between 1979 and 1997, it provides insignificant trends for most regions of the basin. This result indicates that the Yangtze River basin is drying up, the conclusion also reached by a new World Wide Fund for Nature (WWF) study (http://www.asianscientist.com/topnewsLyangtze-river-basin-is-drying-up-wwf-china-2012/).

The MKS test is applied to detect the transition points in the temporal behavior of TWS standardized anomalies based on the annual mean, the wet season mean and the dry season mean, respectively. The definition of wet season and dry season is based on the precipitation climatology of the Yangtze River basin. The Yangtze River basin experiences a distinct wet season from about May to late September or 
early October. The corresponding dry season spans from late September or early October to spring. The summer monsoons contribute most of the wet season precipitation (Harvey and Tang, 2007). As seen in Figure 6.4, generally speaking, the spatially averaged TWS standardized anomaly trends are not significant ( $<95 \%$ confidence level) and not monotonic (i.e. with a transition point) during the period 1979 to 2010. The only transition point during the 32-year period of 1979 to 2010, at which point the TWS standardized anomalies began to decrease sharply, occurred in 2006. This trend reaches the $95 \%$ confidence level in 2010. The transition point occurs one year earlier in the wet season, and two years later in the dry season. In the middle and lower Yangtze reaches, the transition point occurs around 2005 both in the wet and in the dry season. It is noted that there is a significant downward trend in 2009 and 2010 after four years of insignificant decrease. It was the first time this happened since the start of the study in 1979. In the upper Yangtze reaches, the TWS standardized anomalies experience mainly downward trends during the wet seasons of the past 3 decades and increasing trends during the dry seasons. In addition, transition points occur several times (1982, 1989, 1995, 2001, 2005 . . .) and in the period of 1986-1988, the decrease is significant in the wet season in the upper Yangtze reaches. We also examined the transition points through MKS of the TWS standardized anomalies in the middle and lower Yangtze reaches during June-July, and the result is exactly the same as in the wet season, though the TWS standardized anomalies do differ from each other.

As seen in Figures 6.4 and 6.5, the past 6-year period (2005-2010) was the driest period in the Yangtze River basin (especially in the middle and lower reaches) since 1979. This result is quite consistent with the severe drought events documented for the basin by other research. Wei et al. (2012) documented that the Yangtze River basin suffered one of the driest rainy seasons during the 32-year period of 1979 to 2010 in 2005, and Yan et al. (2007) noted that a widespread drought occurred over the southwestern part of the basin that same spring, and that it was the most serious drought since 1979 till 2007. Then the worst drought in more than a century struck southwest China and Sichuan in the summer of 2006, and Dai et al. (2008) showed that the middle and lower Yangtze River reaches suffered the lowest level of the past 50 years during that flood season. In 2007, the area around the Yangtze River suffered a severe drought again. In some places the 
water levels of the river dropped to their lowest levels since records began 142 years ago. The drought was also severe in large areas of the normally wet south. Reservoirs and rivers shrunk and supplies of drinking water fell to alarmingly low levels. However, the extreme drought of 2009/2010 over southwestern Yangtze (including Yunnan, Sichuan and Guizhou) is the driest meteorological event with the lowest percentage rainfall anomaly and the longest rain-free period occurring during a winter season (October-February) in the past 50 years, and also the severest one with the lowest percentage rainfall anomaly since 1880, as documented by Yang et al. (2012).
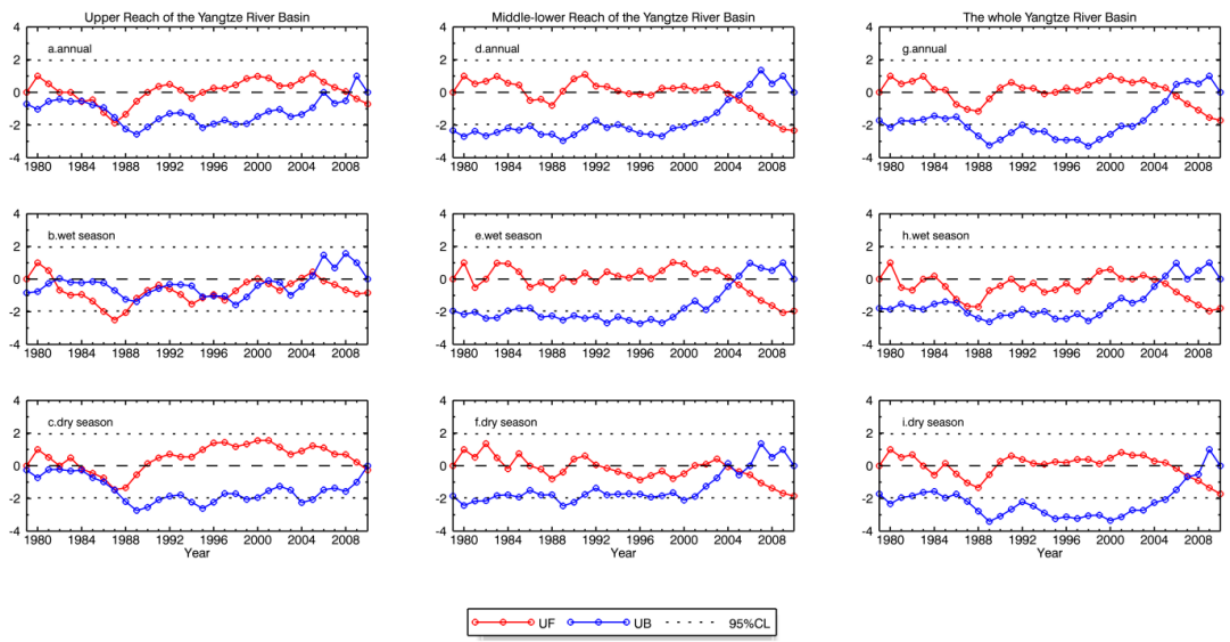

Figure 6.4.The forward (UF, red) and backward (UB, blue) Mann-Kendall statistic rank series for TWS standardized anomalies, per year $(a, d, g)$, per wet season $(b, e, h)$, and per dry season (c, $f, i)$ during the period 1979 to 2010 for the upper reaches $(a, b, c)$, the middle-lower reaches $(d, e, f)$ and the whole $(g, h, i)$ of the Yangtze River Basin(the horizontally dotted lines represent the critical values corresponding to the $95 \%$ confidence level) 

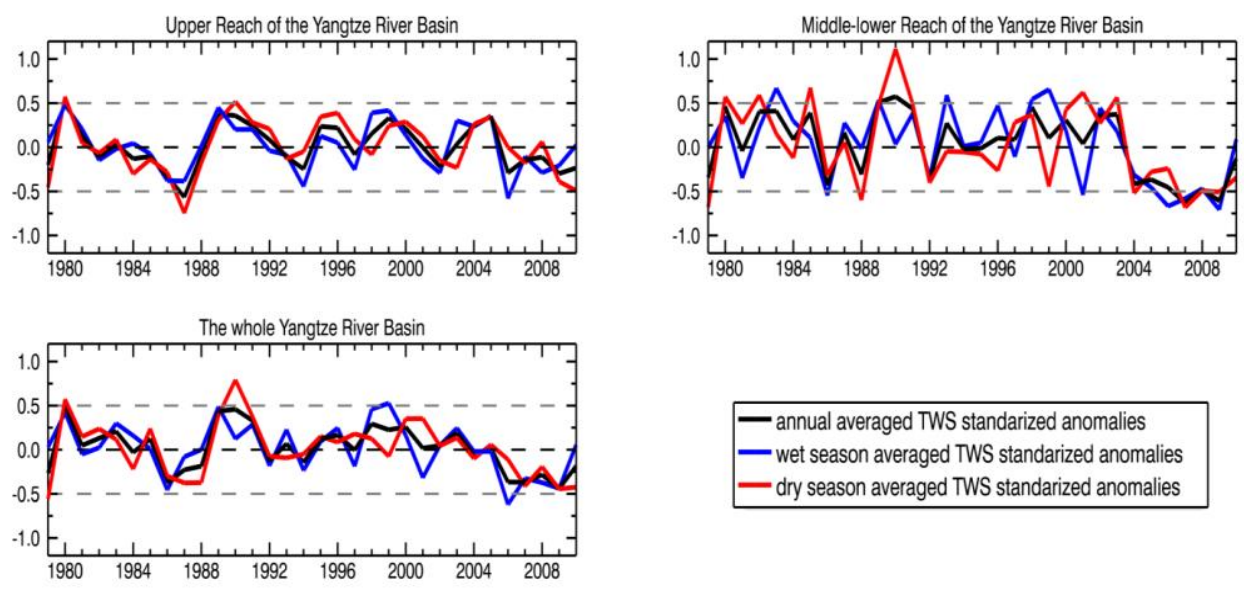

Figure 6.5.The time series of the annual, wet season (May-October) and dry season averaged (November-April) TWS standardized anomalies for the upper Yangtze Reaches, the middle-lower Yangtze reaches and the whole Yangtze River Basin, respectively.

Monthly standardized anomalies of precipitation from ERA-Interim, the GPCC, and PREC/L have been computed and compared to monthly standardized anomalies of TWS (not shown here) to examine possible correlation. The correlation between TWS anomalies from ERA-Interim and precipitation anomalies from ERA-Interim, the GPCC, and PREC/L concerning the Yangtze basin is reasonably high in the wet season $(0.69,0.53$, and 0.49 , respectively), but much lower, especially for GPCC and PREC/L, in the dry season $(0.48,0.21$, and 0.25 , respectively). From a regional perspective, the middle and lower Yangtze reaches exhibited greater agreement between TWS from ERA-Interim and precipitation from the three datasets, than the upper Yangtze reaches did. The notable negative TWS anomalies in the middle and lower Yangtze reaches are in clear agreement with the significant decrease in precipitation seen in the ERAInterim data in the past 6 years; the GPCC and PREC/L data exhibit more gentle negative precipitation anomalies during this period and do not show any special difference to the prior period (not shown here). The differences can also be seen clearly in Figure 3.2, where there is a clear downward shift for ERA-Interim relative to both the GPCC and PREC/L data in the past 6 years. This shift matches the general decline in values relative to the GPCC data for the past decade, which 
may be caused by a too low sea surface temperature (SST) in the ERA-Interim dataset, or fewer stations in the GPCC archive in recent years (Simmons et al., 2010). PREC/L uses fewer gauging stations since the 1990s as well, thus it is difficult to assess the recent huge and sudden drop in the ERA-Interim figures only by comparing them to the GPCC and PREC/L data. However, the dramatic precipitation decrease in the middle and lower Yangtze reaches had been examined by Zhu et al. (2011, Figure 2) over the past decade (2000-2008), and the rainfall anomalies based on a 160-station precipitation dataset of the last 58 years (1951-2008) dropped sharply from positive to negative values around 2004. This pattern of precipitation is consistent with the dramatic decrease of precipitation seen in ERA-Interim data, changing from positive to negative values since 2004 in the middle and lower Yangtze reaches (Figure 3.2), suggesting that the recent drop in precipitation is most likely the biggest contributor to the massive decline in TWS in the middle and lower Yangtze reaches. Ding et al. (2008) pointed out that the recent change in the summer rainfall pattern in the Yangtze River is strongly related to the variability of the East Asian summer monsoon (EASM) through its moisture transport and supply. Zhu et al. (2011) stated that the eastward recession of the western Pacific subtropical high (WPSH) and the significant changes in the global sea surface temperature (SST) are the main causes of the rainfall deficit in the Yangtze River basin since the year 2000. Yan et al. (2007) and Liu et al. (2007) documented that the intensification and westward shift of the WPSH and the easterly anomaly over the northern Indian Ocean are two key causes of the 2005spring drought over southwestern China. The northward shift of the WPSH and the negative snow cover anomaly over the Tibetan Plateau are important contributors to the 2006-summer drought (Zou and Gao, 2007; Li et al., 2009). The extreme drought event of 2009/2010 over southwestern China is associated with the westward extension of the WPSH brought about by the Arabian Sea cyclonic anomaly and the El Nino Modoki event during 2009/2010.

Since ERA-Interim TWS products do not include the impact of anthropogenic activity, such as the TGD and land cover change, in the model structure but rather in the assimilated observations, their effect on the regional climate in the Yangtze River basin is not obvious. However, some studies have tried to demonstrate the extent of human impact on the Yangtze River basin. For example, Dai et al. (2008) 
and Yang et al. (2010) show that the TGD reservoirs could have a direct impact on the intra-annual changes in the downstream Yangtze discharges, leading to a dumping of the seasonal variations in the Yangtze River discharge in the middle and lower reaches. Miller et al. (2005) and Wu et al. (2006) also documented that the land use change associated with the TGD would alter the regional pattern of precipitation, wind, and temperature. It could also impact the hydrological cycle of the river basin, and may lead to changes in the soil-climate interaction, which would probably alter the current dumping effect of soil wetness on the climate variability. As shown in Figures 6.4 and 6.5, the consistent droughts in recent years and the operation of the TGD have occurred simultaneously. In 2003, the water level of the TGR reached $135 \mathrm{~m}$. Coincidently, in 2004, the driest period of the past 32 years began for the middle and lower Yangtze. Also, the whole basin suffered an abrupt change in 2006, when the TGR raised its water level from 135 to $156 \mathrm{~m}$. This coincidence is very striking and may imply the possible connection between the TGD and the consistent droughts in recent years, even though there has been no irrefutable evidence to prove that the TGR is responsible for the extremely driest period that has occurred in the past several years, as the TGD has only been in operation for a short period. Apart from the TGR, numerous other reservoirs within the Yangtze catchment together reached $200 \mathrm{~km}^{3}$ (Yang et al., 2005), more than five times the storage capacity of the TGR. The impact of these reservoirs on the TWS should not be ignored. The Yangtze basin has witnessed remarkable changes in land use and cover induced by high population density and rapid but uneven economic growth (Long et al., 2006; Yin et al., 2010). These changes might alter the soil properties and soil-climate interactions, probably having great influence on the TWS and runoff distribution. It should be pointed out that the ERA-Interim TWS could contain significant uncertainties, as it relies heavily on satellite observations and modeling. Further investigation and analysis is needed to assess the significant impact of human activity on the TWS of the Yangtze River basin.

\subsection{Conclusions}

This study analyzes the spatial and temporal variations of the TWS in the Yangtze River basin during the period 1979 to 2010 based on ERA-Interim datasets. Linear 
regression and the MK test have been used to detect trends and the significance of trends in TWS for each pixel in the whole basin. In addition, the MKS test has been used to detect transition points in the temporal series of the spatially averaged TWS annual standardized anomalies in the upper, middle/lower, and whole basin, respectively. We conclude that the TWS variations over the Yangtze River basin during the period 1979 to 2010 have the following characteristics:

1) Most of the Yangtze basin exhibits the highest positive TWS anomalies during June-July, mainly due to the Meiyu rain event. This intensified rainfall not only results in very high positive TWS anomalies, but probably also leads to high runoff anomalies, causing floods across the basin.

2) The Yangtze River basin is drying up, especially since the year 1998. The TWS variation is strongly correlated to the precipitation variation derived from ERA-Interim, the GPCC and PREC/L data, especially during the wet season and in the middle and lower reaches, suggesting the TWS variation is mainly controlled by precipitation.

3) In the middle and lower Yangtze reaches, the TWS behavior changed abruptly and started to decrease in 2004. Coincidently, the TGR started impoundment in 2003. The year 2006 is detected as only transition point, initiating the TWS to suffer high negative anomalies, while the TGR raised its water level from 135 to $156 \mathrm{~m}$ that same year. Due to the short period of the TGD being in operation, it is hard to prove a link between the TGR and the consistent drought in recent years, but the coincidence suggests a possible connection.

4) The past 6-year period (2005-2010) was the driest period in terms of TWS in the Yangtze River basin (especially in the middle and lower Yangtze reaches) since 1979. This is mainly the result of a dramatic decrease in precipitation. 


\section{CHAPTER 7}

Human-induced Changes in Terrestrial Water Storage of the Yangtze River Basin Estimated from GRACE Satellite Data and Land Surface Model Simulations 



\section{Chapter 7 Human-induced changes in terrestrial water storage of the Yangtze River basin estimated from GRACE satellite data and land surface model simulations}

\section{This chapter is based on:}

Huang, Y., M. S. Salama, M. S. Krol, Z. Su, A. Y. Hoekstra, Y. Zeng, and Y. Zhou, 2015: Estimation of human-induced changes in terrestrial water storage through integration of GRACE satellite detection and hydrological modeling: A case study of the Yangtze River basin. Water Resources Research, 51, 8494-8516.

\subsection{Abstract}

Quantifying the human effects on water resources plays an important role in river basin management. In this study, we proposed a framework, which integrates the Gravity Recovery and Climate Experiment (GRACE) satellite estimation with macro-scale hydrological model simulation, for detection and attribution of spatial terrestrial water storage (TWS) changes. In particular, it provides valuable insights for regions where ground-based measurements are inaccessible. Moreover, this framework takes into account the feedback between land and atmosphere, andinnovatively puts forward several suggestions (e.g. study period selection, hydrological model selection based on soil moisture-climate interactions) to minimize the uncertainties brought by the interaction of human water use with terrestrial water fluxes. We demonstrate the use of the proposed framework in the Yangtze River basin of China. Our results show that, during the period 2003-2010, the TWS was continually increasing in the middle and south eastern reaches of the basin, at a mean rate of about $3 \mathrm{~cm} \mathrm{yr}^{-1}$. This increment in TWS was attributed to anthropogenic modification of the hydrological cycle, rather than natural climate variability. The dominant contributor to the TWS excess was found to be intensive surface water irrigation, which recharged the water table in the middle and south 
eastern parts of the basin. Water impoundment in the Three Gorges Reservoir (TGR) is found to account for nearly $20 \%$ of the human-induced TWS increment in the region where the TGR is located. The proposed framework gives water managers/researchers a useful tool to investigate the spatial human effects on TWS changes.

\subsection{Introduction}

IPCC (2012) has documented evidence, based on observations gathered since 1950, of change in the extremes of, for instance, precipitation and discharge. There is medium confidence that some regions of the world have experienced more intense and longer droughts, whereas, in some regions, there have been statistically significant trends concerning the number of heavy precipitation events. This may relate to indirect human influence on water resources, referring mainly to the effects of anthropogenic changes in climate, which are, for instance, associated with greenhouse gas emissions. Direct human influence is attributed to human alteration of river basins, including but not limited to hydroelectricity generation, irrigation, groundwater abstraction, and land use and cover change (LUCC). On the one hand, human activity can have a large amount of benefits. For example, man-made reservoirs can prevent floods, ease droughts, and generate electricity; irrigation can increase crop production. On the other hand, they commonly affect the distribution, quantity, and chemical quality of water resources, and are therefore very likely to have adverse effects. For instance, it is prevalent that increasing water withdrawal worsens water scarcity conditions in semiarid and arid regions (e.g. Pakistan, India, northeastern China, the Middle East, and North Africa), increasing uncertainties in sustainable food production and economic development (Hanasaki et al., 2008b; Kummu et al., 2010; Vörösmarty et al., 2010; Wada et al., 2011b). Moreover, water impoundment, for instance, in the Three Gorges Reservoir (TGR), can cause a large mass redistribution of water in a short period, likely leading to groundwater recharge and contamination (Wang et al., 2011). Therefore, quantifying the relative effects of natural and human influences on river basins is essential for water management.

In recent decades, a number of macro-scale hydrological models have been developed and continuously improved, in order to analyze the effects of human 
actions on water resources at large scales. Alcamo et al. (2003a, b) developed the global water resources and use model WaterGAP, and estimated the water withdrawals and availability at a global scale. Döll et al. (2009) used the WaterGAP Global Hydrological Model (WGHM) to estimate and analyze globally the river flow alterations due to water withdrawals and reservoirs. Furthermore, Döll et al. (2012) used WGHM to estimate the impact of water withdrawals on continental water storage variations. Hanasaki et al. (2008a, b) developed the integrated water resources assessment model $\mathrm{H} 08$ to stimulate both natural and anthropogenic flows of water globally. Due to the fact that few of these efforts had addressed the issue of human impacts on the terrestrial water cycle by explicitly representing them within the framework of land surface models (LSMs), Pokhrel et al. (2011) developed an integrated modeling framework (MATSIRO) for assessing the impact of anthropogenic water regulation on surface energy balance. Furthermore, Wada et al. (2014) substantially improved the PCR-GLOBWB model (Wada et al., 2010, 2011a, b; van Beek et al., 2011) by, for instance, explicitly taking into account the mutual feedback between water supply and demand. These models are very valuable to analyze the human perturbation on global water resources; however, they have their own deficiencies and may contain large uncertainties at a regional scale. For example, Pokhrel et al. (2011) pointed out that there are certain limitations in their model, including the lack of explicit representations of water table dynamics, water diversions, and evaporation from large reservoirs. Moreover, WGHM does not consider the mutual feedback between human water use and terrestrial water fluxes. In addition, as documented by Wada et al. (2014), the improved PCR-GLOBWB model is limited to semiarid and arid regions, and unable to reproduce changes in the distribution within countries.

Several studies have tried to analyze, at a regional scale, the effects of human actions on water resources, by comparing the simulated naturalized situation of the hydrological system with the real situation (Barco et al., 2010; Lorenzo-Lacruz et al., 2010; Mair and Fares, 2010). Furthermore, van Loon and van Lanen (2013) proposed an observation-modeling framework to make the distinction between water scarcity (human causes) and drought (natural causes). This framework gives water managers a useful tool to separate natural and human effects on the hydrological system. However, there are some limitations and issues. First of all, it 
cannot be used in data-scarce regions, because the basic requirements of the framework are the observed data: meteorological data for the entire period, hydrological data for the period without human influence, and hydrological data for the period with human influence. Second, the framework is not suitable for large basins with high geographic diversity, because it does not provide information about changes in spatial distribution of hydrological variables. One possible scenario is that the spatial human effects are cancelled out at basin scale, which gives water managers the illusion of an absence of human effects on the hydrological system. Third, strategies are needed to address the uncertainties introduced by the feedback between human water use and terrestrial water fluxes. These uncertainties include two main parts. The first part is that the observed meteorological data are influenced by human actions. For instance, Pokhrel et al. (2011), Lo and Famiglietti (2013), and others have documented that irrigation alters the surface energy balance, influencing local weather and climate. The second part is the impact of climate variability on human actions. For instance, climate variability may change irrigation water demand, and consequently affect water withdrawal, allocation and consumption (Thomas, 2008; Wisser et al., 2008).

Emerging advances in hydrologic remote sensing fill the gaps in data availability and water monitoring. The Gravity Recovery and Climate Experiment mission (GRACE) (Tapley et al., 2004b), comprising twin satellites launched in March 2002, offers a valuable tool to measure temporal and spatial terrestrial water storage (TWS) variations. The GRACE data have been used to validate macro-scale hydrological models (e.g. Döll et al., 2009; Pokhrel et al., 2011), or applied to estimate human-induced changes in groundwater storage (e.g. Rodell et al., 2009; Feng et al., 2013; Voss et al., 2013). However, the GRACE data are mainly used to estimate groundwater depletion or water impoundment in reservoirs rather than groundwater recharge. This may be attributed to two factors. First, the groundwater recharge caused by, for instance, large scale irrigation is more complex than groundwater depletion caused by groundwater pumping. The reason for this is that irrigation is influenced by both climate and humans, and may alter surface energy balance and hence influence regional climate. Second, the human-induced groundwater recharge at a large basin scale may not be significant enough to attract the attention of water managers/researchers, and since GRACE 
data may contain significant errors for relatively small domains, the majority of GRACE applications are still at large scales. The GRACE based groundwater depletion studies did not explicitly consider the uncertainties generated by climate influences on their groundwater depletion estimates, an issue that will be addressed in this chapter.

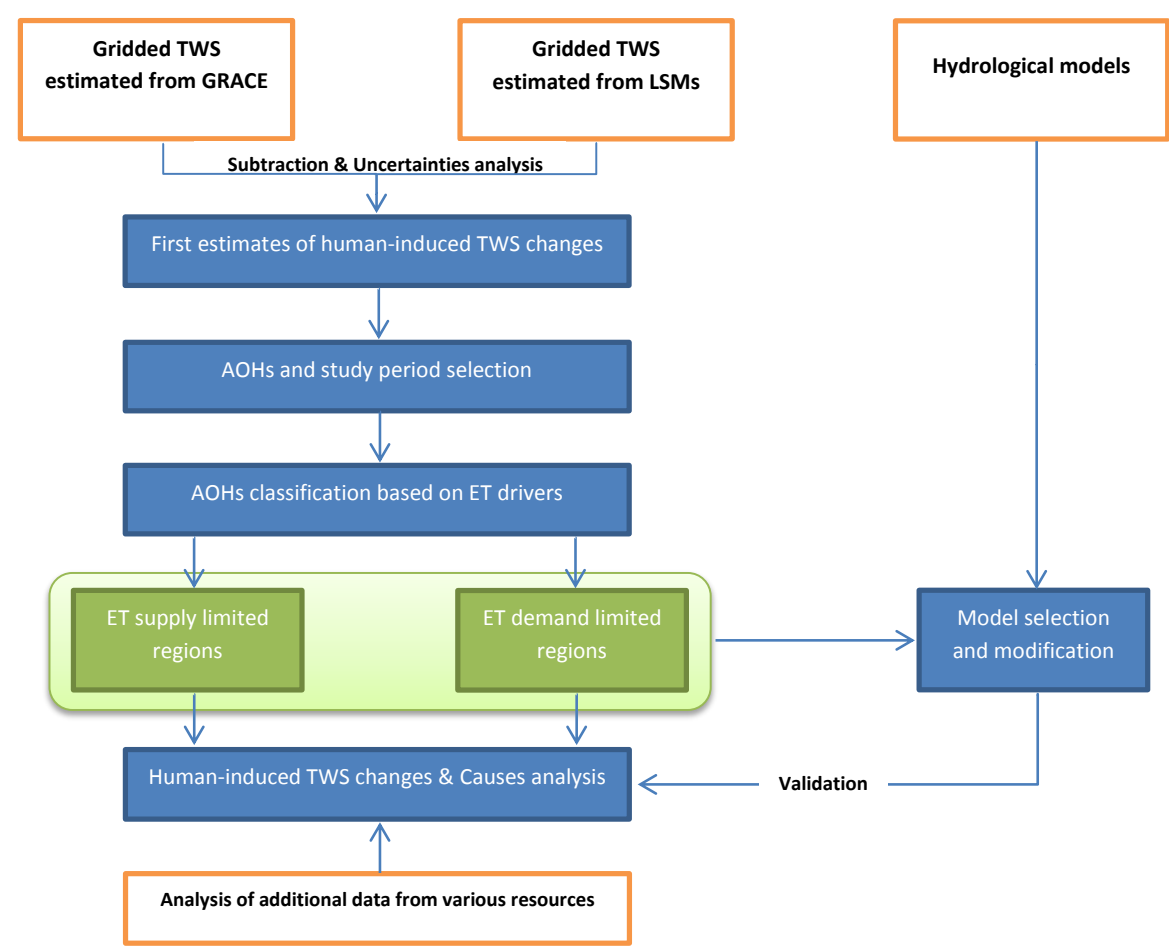

Figure 7.1.The framework for detection and attribution of spatial TWS changes at basin scales.

Here, we propose a framework, which combines the virtues of GRACE satellite estimation and hydrological model simulation, for detection and attribution of spatial TWS changes. The advantages of this framework are listed as follows. First, the observational data requirement is low, as publicly available datasets are mainly used. Second, it takes into account the feedback between human water use and climate variation. Third, it encourages water managers to, especially for large river basins, locate and analyze regions with observed human effects (ROHs) and further explore the causes, rather than focus solely on basin-average analysis. This 
framework integrates GRACE satellite detection with macro-scale hydrological modeling, and gives water managers and researchers a useful tool to investigate the spatial human effects on TWS in various climate regimes.

This framework is illustrated by its application in the Yangtze River basin. The GRACE data have rarely been applied in a water rich basin to estimate spatial effects of human-induced changes on TWS mainly caused by large-scale irrigation. The Yangtze River basin experiences intensive human activity. For instance, the basin has been documented as one of the areas with the highest irrigation density in the world (Siebert et al., 2005), and intensive irrigation could also have substantial impact on TWS changes (Döll et al., 2012; Zhang et al., 2015). Although the Yangtze River basin was, in the above-mentioned previous studies, routinely taken as one of the major basins in the world for global water assessment, the human effects on the basin were rarely highlighted or validated by GRACE users and macro-scale hydrological modelers. This may be due to the difficulty of resolving the effects of human-induced TWS change on a sub basin scale.

In this chapter, we first, in section 7.3, explain the framework for detection and attribution of spatial TWS changes. The application of the framework to the case study area is illustrated in section 7.4 and discussed in section 7.5. Finally, the conclusion is drawn in section 7.6.

\subsection{The framework for detection and attribution of spatial TWS changes}

The framework designed for detection and attribution of spatial TWS changes is depicted in Figure 7.1. It integrates two fully independent methodologies, using (1) GRACE satellite data combined with LSMs and (2) macro-scale hydrological modeling, to cross-check the estimated human-induced TWS changes.

The left side of Figure 7.1 shows the method, subtracting LSM estimates from GRACE-observed TWS values, to preliminarily estimate the spatial effects of human-induced changes on TWS (equation (7.1)), as GRACE data detect TWS changes affected by both climate variability and human activities, whereas LSM simulations represent the climate-related TWS changes. 


$$
T W S_{\text {human }}=T W S_{\text {GRACE }}-T W S_{\text {natural }},
$$

where $T W S_{\text {human }}$ is the estimated human-induced TWS variation, $T W S_{\mathrm{GRACE}}$ is the GRACE-derived TWS variation, and $T W S_{\text {natural }}$ is the natural part of TWS variation estimated from the LSM. TWS is expressed as equivalent water height (EWH) (cm).

The inter-annual trend is computed, at each grid, by means of linear regression of the annual mean values of the estimated human-induced TWS. It should be noted that the estimated inter-annual trend does not explicitly consider seasonal variations, and hence the influence of seasonal variations on the estimated trends needs to be discussed. Uncertainty in inter-annual human-induced TWS trend is estimated by propagating errors from the GRACE-observed TWS trend and the LSM simulated TWS trend.

$$
\sigma_{h_{-} t}=\sqrt{\sigma_{\mathrm{g}_{\mathrm{t}}}{ }^{2}+\sigma_{\mathrm{l}_{-}}{ }^{2}}
$$

where $\sigma_{h_{-} t}, \sigma_{\mathrm{g}_{-} \mathrm{t}}$ and $\sigma_{\mathrm{l}_{\mathrm{t}} \mathrm{t}}$ are the uncertainty for human-induced TWS trend, GRACE-based TWS trend and LSM simulated TWS trend, respectively. These values are provided by GRACE land products.

Uncertainty in GRACE-derived TWS trend, at each pixel, is computed in two steps: Firstly, we estimate the monthly error of GRACE-derived TWS by using measurement error and leakage error as:

$$
\sigma_{g}=\sqrt{\sigma_{\mathrm{l}}^{2}+\sigma_{\mathrm{m}}^{2}}
$$

where $\sigma_{\mathrm{g}}$ is the error for GRACE-based TWS, $\sigma_{1}$ is the leakage error, and $\sigma_{\mathrm{m}}$ is the measurement error.

Secondly, we propagate the monthly error of GRACE-observed TWS onto the least-squares-estimated inter-annual trend based on Morrison (2014) as follows:

$$
\sigma_{g_{-} t}=N_{\text {months }} * \sigma_{g} \sqrt{n\left[n \sum_{i=1}^{n} x_{i}{ }^{2}+\left(\sum_{i=1}^{n} x_{i}\right)^{2}\right] /\left[n \sum_{i=1}^{n} x_{i}{ }^{2}-\left(\sum_{i=1}^{n} x_{i}\right)^{2}\right]},
$$


where $x_{i}, n$, and $N_{\text {months }}$ are the sequential data values, the dataset record length of the monthly GRACE-based TWS anomalies, and the number of months for one year, respectively.

Moreover, uncertainty in LSM simulated TWS trend $\left(\sigma_{l_{-}}\right)$, at each pixel, is estimated as the standard deviations of the trends computed from LSMs, as used by Famiglietti et al. (2011) and Voss et al. (2013) in similar cases:

$$
\sigma_{l_{-} t}=\sqrt{\sigma_{\mathrm{e}_{-}}{ }^{2}+{\sigma_{\mathrm{r}_{\mathrm{t}}}}^{2}},
$$

where $\sigma_{\mathrm{e}_{-} \mathrm{t}}$ and $\sigma_{\mathrm{r}_{-} \mathrm{t}}$ are the uncertainties for the employed LSM simulated TWS trend and the referenced LSM simulated TWS trend, respectively.

After the preliminary estimates of human-induced TWS changes, several ROHs are selected for further examination. This does not only allow us to explore specific causes at regional scales, but also to reduce uncertainties at grid scales, as increasing the size of the region when calculating a regional average generally reduces errors and uncertainties considerably (Landerer and Swenson, 2012). The selection of ROHs is primarily based on the extent of the discrepancy between the GRACE-derived TWS and LSM simulations, hydro-meteorological conditions, and types of human activity. The selection of the study period is also an important step in the framework. We selected the period during which the inter-annual and seasonal variability in the estimated TWS from the LSM are rather stationary (natural 'stationary' period) for the study. By selecting this period mathematical artefacts caused by the subtraction of a large negative LSM simulated TWS trend from the GRACE-derived one can to a certain extent be avoided, and the uncertainties caused by the feedback between human water use and terrestrial water fluxes reduced. More specifically, for the period when the climate is relatively stationary, the change in irrigation water demand related to climate variability is small, and hence the estimates from the method, subtracting the LSM simulated TWS from the GRACE derived TWS, largely exclude the uncertainties caused by the influence of climate variability on human activities (e.g. irrigation, water withdrawal, water consumption).

From a historical perspective, the satellite-based estimates of human-induced TWS are validated by ground-based measurements such as water level measurements. 
However, these field data are available for only a few regions, and rarely for periods of more than a few years. Besides, in situ observations are point measurements, and not always representative for larger spatial domains (Famiglietti et al., 2008). Therefore, modeling of the human effects on TWS changes is a valuable alternative, especially for data-limited regions (Figure 7.1, the first approach for validation). For the regions with in situ measurements of water levels, it is recommended to apply data assimilation, which combines the virtues of in situ measurements and model simulation of the human effects on TWS, to validate the GRACE satellite based estimates of human-induced TWS changes (Figure 7.1, the second approach for validation).

As models have their own deficiencies and limitations, model selection for validation in individual regions is no trivial matter. In order to minimize the uncertainties caused by the feedbacks between human activities and climate variation, information on evapotranspiration (ET) drivers is used to select a hydrological model for validation of ROHs or a certain ROH. Jung et al. (2010) and Seneviratne et al. (2010) have documented that there is evidence from observations and modeling that, in terms of ET-soil moisture (SM) coupling strength, regions can be roughly classified into two categories: ET supply limited (SM-limited) regions and ET demand limited (energy-limited) regions. In ET supply limited regions, ET is largely governed by the availability of SM, whereas, in ET demand limited regions, ET is mainly controlled by net radiation and not sensitive to SM. As a result, models that consider the effects of human actions on surface energy balance and the potential climate feedbacks should be chosen for ET supply limited regions, since human actions (e.g. irrigation area extension, reservoirs construction) largely change SM, and hence have non-negligible impact on regional climate. On the other hand, such models may not perform well in ET demand limited regions, because the realistic representations of other processes are more important than land-atmosphere coupling. It should be noted that some regions may switch between ET demand limited and ET supply limited regimes over the course of the year (e.g. Ryu et al., 2008), and may also be dependent on land cover (e.g. Zhang et al., 2001; Zaitchik et al., 2006). Since we focus on interannual trends of $\mathrm{ROH}$ in this framework, ROHs are classified according to the ET regime dominant during the study period, for which the Supplementary Figure 2 
of Jung et al. (2010) and Figure 8 of Seneviratne et al. (2010) offer valuable information.

Previous studies (e.g. Seneviratne and Stockli, 2008; Teuling et al., 2009) have illustrated that drivers of ET vary with climate regimes, with a relatively high correlation coefficient of ET and SM in dry regimes, next to a relatively high correlation coefficient of ET and radiation in wet regimes. Despite regional variations, human actions are associated with climate regimes, and hence with ETSM coupling strength. A large amount of groundwater withdrawal tends to occur in dry regions, whereas surface water is more likely to be predominantly used in wet regions. Therefore, groundwater depletion usually occurs in ET supply limited regions, whereas groundwater recharge caused by surface water irrigation is more likely to take place in ET demand limited regions. These assumptions can be crosschecked in this framework, and further supported by data (e.g. discharge, lake volumes, vegetation cover fraction) from various sources.

\subsection{Application of the framework to the Yangtze River basin}

\subsubsection{Preliminary estimates of human-induced TWS variations}

We preliminarily applied a linear regression model, at each pixel, to the annual mean values of the satellite estimated human-induced TWS anomalies. Figure 7.2 illustrates the inter-annual trends of human-induced TWS variations with the unit of $\mathrm{cm} \mathrm{yr}^{-1}$ EWH for the period 2003-2010. The trends of human-induced TWS variations obtained from GRACE and Noah-MP (Figure 7.2a) show a very similar spatial pattern to those computed from GRACE and ERA-Interim/Land (Figure $7.2 b$ ). This is because they stem from the trends of the GRACE data (Figure 7.2c), as both Noah-MP and ERA-Interim/Land simulations have no significant trends during the study period. The human-induced TWS shows sharp positive trends in the middle and lower reaches, with a maximum of more than $5 \mathrm{~cm} \mathrm{yr}^{-1}$, while a negative trend is observed in the upper reaches. Figure 7.3 depicts the uncertainties of the human-induced TWS trends of the study area, which are generally larger in the upper reaches of the basin than in the middle and lower reaches. Those uncertainties were estimated by propagating errors from the GRACE-observed 
TWS trend and LSM simulated TWS trend (equation (7.2)). The uncertainties in GRACE-derived TWS trends were computed according to equations (7.3) and (7.4). The uncertainties in Noah-MP, ERA-Interim/Land simulated TWS trends were respectively estimated as the standard deviations of the trends computed from the used LSM and from GLDAS-Noah (equation (7.5)).
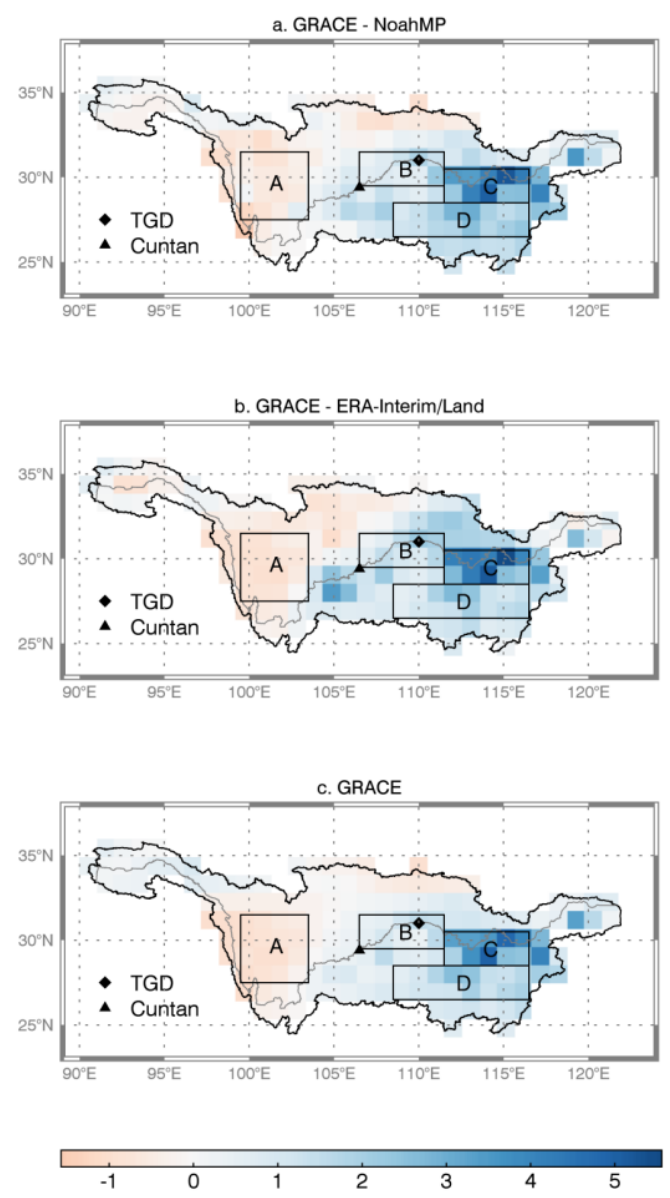

Figure 7.2. Inter-annual trends of (a) estimated human-induced TWS from GRACE and Noah-MP simulations, (b) estimated human-induced TWS from GRACE and ERA-Interim/Land reanalysis data, and (c) estimated TWS from GRACE data, with the spatial resolution of $1^{\circ}$ in the Yangtze River basin for the period 2003-2010; the gray line represents the Yangtze mainstream. The diamond and triangle represent the location of the TGD and the Cuntan hydrological station, respectively. Squares A, B, C and $\mathrm{D}$ represent the selected regions. Units: $\mathrm{cm} \mathrm{yr}^{-1}$ 

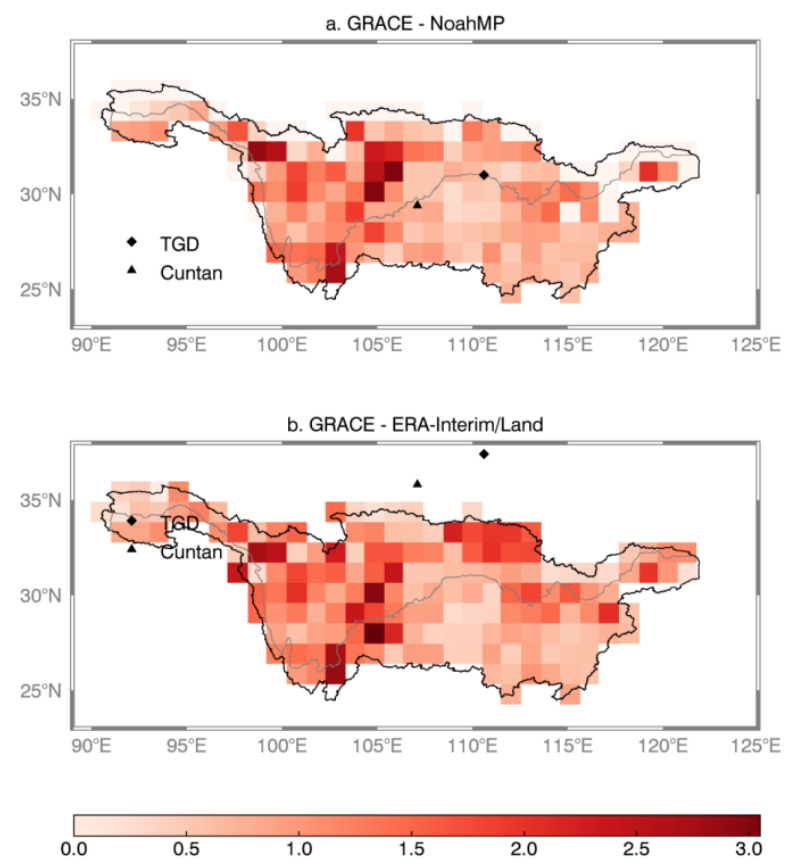

Figure 7.3.Uncertainties in the annual trends of human-induced water storage based on (a) GRACE and Noah-MP simulations, (b) GRACE and ERA-Interim/Land reanalysis data in the Yangtze River basin; Units: $\mathrm{cm} \mathrm{yr}^{-1}$

\subsubsection{ROHs and study period selection}

Based mainly on the growth rates of the first estimated human-induced TWS changes, we defined the Regions A, B, C and D as ROHs for further examination (Figure 7.2). Region A is located in the upper reaches of the Yangtze River basin, while Regions B, C and D are in the middle and lower reaches (Figure 2.2). It should be noted that Region B includes the hydraulic basin of China's TGR. The positive GRACE-observed TWS trend of Region B, thus, was affected by the TGR water impoundment (Wang et al., 2011). Figure 7.4 shows the spatially averaged monthly time series of the estimated human-induced TWS anomalies in Regions A, $\mathrm{B}, \mathrm{C}$, and D. It depicts that, interestingly, the largest human-induced TWS increase occurred in Region C, with a mean rate of $3.7 \pm 0.8 \mathrm{~cm} \mathrm{yr}^{-1}$ based on GRACE and 
Noah-MP, and a rate of $3.9 \pm 1.1 \mathrm{~cm} \mathrm{yr}^{-1}$ based on GRACE and ERA-Interim/Land, respectively. Regions $\mathrm{B}$ and $\mathrm{D}$ experienced smaller water increment during the period 2003-2010. In contrast, there is a small negative trend in Region A. Also shown in Figure 7.4 is that there is no significant trend in the Noah-MP or ERAInterim/Land simulated TWS. Therefore, the period 2003-2010 can be used as study period. Besides, the seasonal variability of the estimated human-induced TWS changes is rather stationary (Figure 7.4), indicating that, during 2003-2010, seasonal variations have little influence on the computed linear trends.
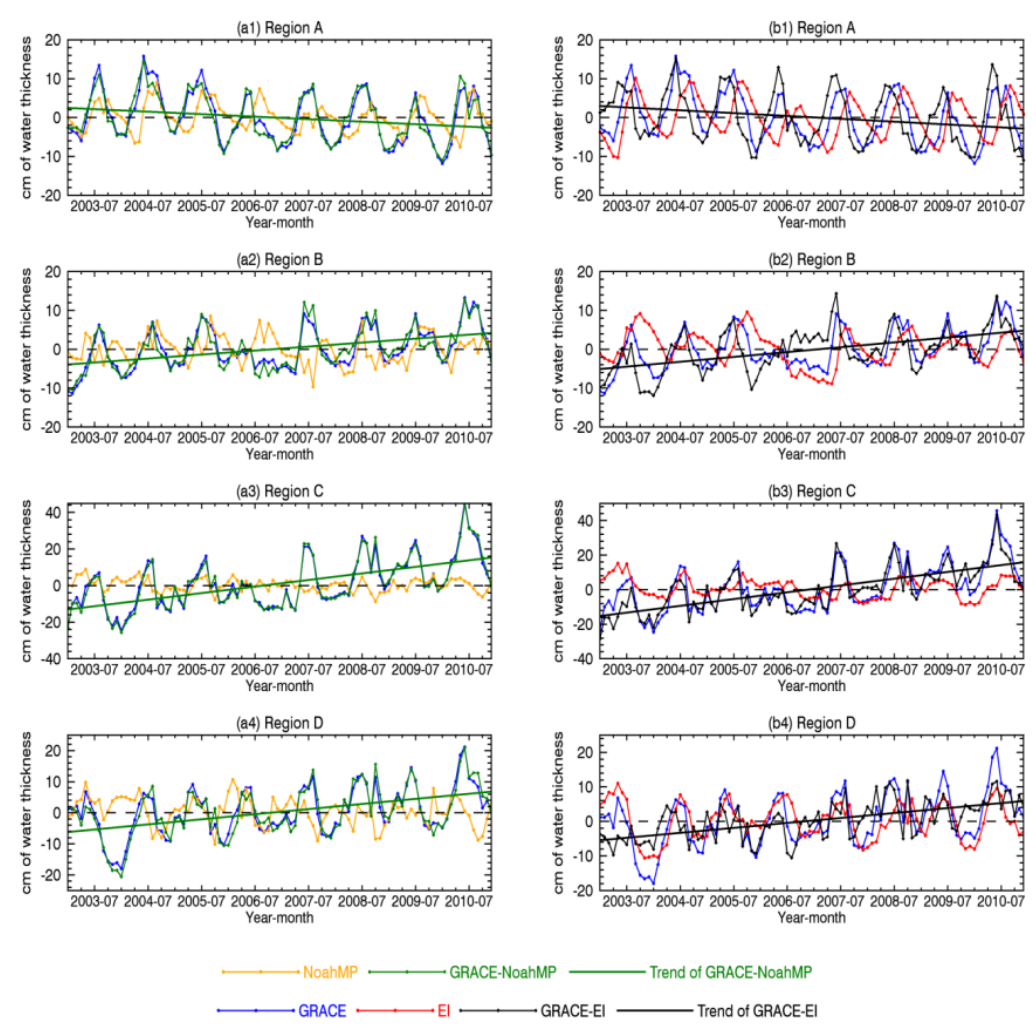

Figure 7.4. Spatially averaged monthly time series of GRACE-observed TWS (blue star curve), NoahMP estimated TWS (orange circle curve), ERA-Interim/Land (EI) estimated TWS (red circle curve), human-induced TWS (green/black diamond curve) variations (as anomalies), and the best-fit linear human-induced TWS trend (green/black line) for Regions A to D during the period 2003-2010. The trends of human-induced TWS variations from GRACE and Noah-MP, in Regions A, B, C, and D, are $0.5 \pm 0.5,1.1 \pm 1.1,3.7 \pm 0.8$, and $1.9 \pm 0.7 \mathrm{~cm} \mathrm{yr}^{-1}$, and from GRACE and ERA-Interim/Land are $-0.7 \pm 1.0$, $1.3 \pm 0.6,3.9 \pm 1.1$ and $1.4 \pm 0.6 \mathrm{~cm} \mathrm{yr}^{-1}$, respectively. 


\subsubsection{ROHs classification based on ET drivers}

Jung et al. (2010) and Teuling et al. (2009) have estimated the drivers of ET based on model and observational data. Seneviratne et al., (2010) have explicitly explained land-atmosphere coupling, the processes controlling ET, and the existence of distinct ET regimes. According to these previous studies, the Yangtze River basin, especially the middle and lower reaches, has consistently been diagnosed as an ET demand limited regime. This is not surprising, because the climate of the Yangtze River basin is predominantly humid, creating a sufficiently high SM level.

\subsubsection{Validation}

Due to the very weak coupling strength between SM and ET, the human activities that change SM (e.g. irrigation) have little impact on ET in the ET demand limited regime. Therefore, the models that consider the feedbacks between land and atmosphere do not have particular advantages. MATSIRO and PCR-GLOBWB 2.0 are two of the most advanced models that take into account the feedbacks between land and atmosphere. However, MATSIRO lacks explicit representations of water table dynamics (Pokhrel et al., 2011), and the assumption of PCR-GLOBWB 2.0, using the fraction of daily accumulated base flow to simulate allocation of surface water and groundwater to satisfy the water demands, is unrealistic in humid regions where people predominantly rely on surface water resources (Wada et al., 2014). Additionally, the impact of irrigation return flow was neglected. Therefore, neither is suitable to use for validation in the Yangtze River basin.

In this case study, we select the method of Döll et al. (2012) to quantify the impacts of surface water and groundwater withdrawal and consumption on TWS variations. This is due to the fact that, although it does not consider the feedback between human water use and terrestrial water fluxes, or equate water demand with either water withdrawals or consumptive water use, it uniquely estimates water withdrawals according to source, for instance, accounting for the difference between water withdrawals from groundwater and water withdrawals from surface water. For a humid basin like the Yangtze, this hydrological model is therefore suitable for evaluating the impacts of human water use on TWS. 
As Döll et al. (2012) concentrated on the impact of water use on seasonal variations in TWS rather than trends in the global-scale analysis, and the estimates were based on the data during the period 1998-2002, their modeling results cannot be used to validate the estimated human-induced TWS changes from GRACE and LSMs in this case study. In addition, according to the officially released water use data, water withdrawals are generally used for four sectors: domestic, manufacturing, irrigation and environment, which are different from the water use sectors defined in Döll et al. (2012). We therefore modified the equations (1) and (3) of Döll et al. (2012), and recomputed the impact of groundwater and surface water use on groundwater and surface water storages.

Water withdrawals for all sectors and sources result in return flow (WU-CU) to surface water. It is assumed that the return flow of the irrigation water withdrawn from either surface water or groundwater partly runs off directly to surface water bodies, while the other part recharges groundwater before running to surface water bodies. The water withdrawals for other uses are assumed to directly flow into surface water even if the water source is groundwater. Therefore, the surface and groundwater storages are artificially changed, respectively, by net abstraction of surface water $N A_{s}$ and net abstraction of groundwater $N A_{g}$, which are computed as follows:

$$
\begin{gathered}
N A_{s}=\left[C U_{s d}+C U_{s e}+C U_{s m}+W U_{s i}\right]-\left[\left(1-f_{r g i}\right)\left(W U_{g i}-C U_{g i}+W U_{s i}-C U_{s i}\right)+\right. \\
\left.\left(W U_{g d}-C U_{g d}+W U_{g m}-C U_{g m}+W U_{g e}-C U_{g e}\right)\right], \\
N A_{g}=W U_{g}-\left[f_{r g i}\left(W U_{g i}-C U_{g i}+W U_{s i}-C U_{s i}\right)\right]
\end{gathered}
$$

where $N A$ is net abstraction $\left(\mathrm{km}^{3} \mathrm{yr}^{-1}\right) ; W U$ is withdrawal use $\left(\mathrm{km}^{3} \mathrm{yr}^{-1}\right) ; C U$ is consumptive use $\left(\mathrm{km}^{3} \mathrm{yr}^{-1}\right)$; $\mathrm{f}_{\mathrm{rgi}}$ is groundwater fraction of return flow from irrigation $(W U-C U)$, and of the subscripts $g$ represents groundwater, $s$ represents surface water, $d$ represents domestic, $e$ represents environment, $i$ represents irrigation, and $m$ represents manufacturing. It should be noted that net abstractions from groundwater and net abstractions from surface water can be positive or negative. Positive values indicate water storage losses, whereas negative values indicate storage gains. The sum of $N A_{s}$ and $N A_{g}$ is equal to consumptive water use. 
Due to the unavailability of water withdrawal and consumption data for some sectors, only $N A_{s}$ for irrigation is calculated in this study.

Irrigation $C U$ is computed by multiplying irrigation $W U$ by irrigation water use efficiencies. We use the default values of irrigation water use efficiencies, 0.378 for surface water use and 0.7 for groundwater use, implemented in WaterGAP for China.

Groundwater recharge is highly dependent on artificial drainage, which causes water to bypass the groundwater store, thus the groundwater fraction $f_{r g i}$ of return flow is calculated as a function of the fraction of irrigated area that is artificially drained $f_{d}$ :

$$
f_{\text {rgi }}=0.8-0.6 f_{d}
$$

where the values 0.8 and 0.6 are taken from Döll et al. (2012). In this study, the spatial yearly return flows are computed by weighting the total yearly return flows from irrigation $(W U-C U)$ with the irrigation area percentage, due to a lack of detailed spatial distribution of water supply and consumption data in the study area (Table 7.1).

As shown in Table 7.1, a huge amount of water (about $180 \times 10^{9} \mathrm{~m}^{3}$ ) is used in the Yangtze River basin every year, and approximately $95 \%$ of the water supply originates from surface water. Water used for irrigation takes up a large portion of the total water supply, accounting for more than $50 \%$ (around $94.8 \times 10^{9} \mathrm{~m}^{3}$ ) of total water use, while the rest of the water supply is used for domestic, manufacturing, etc. 

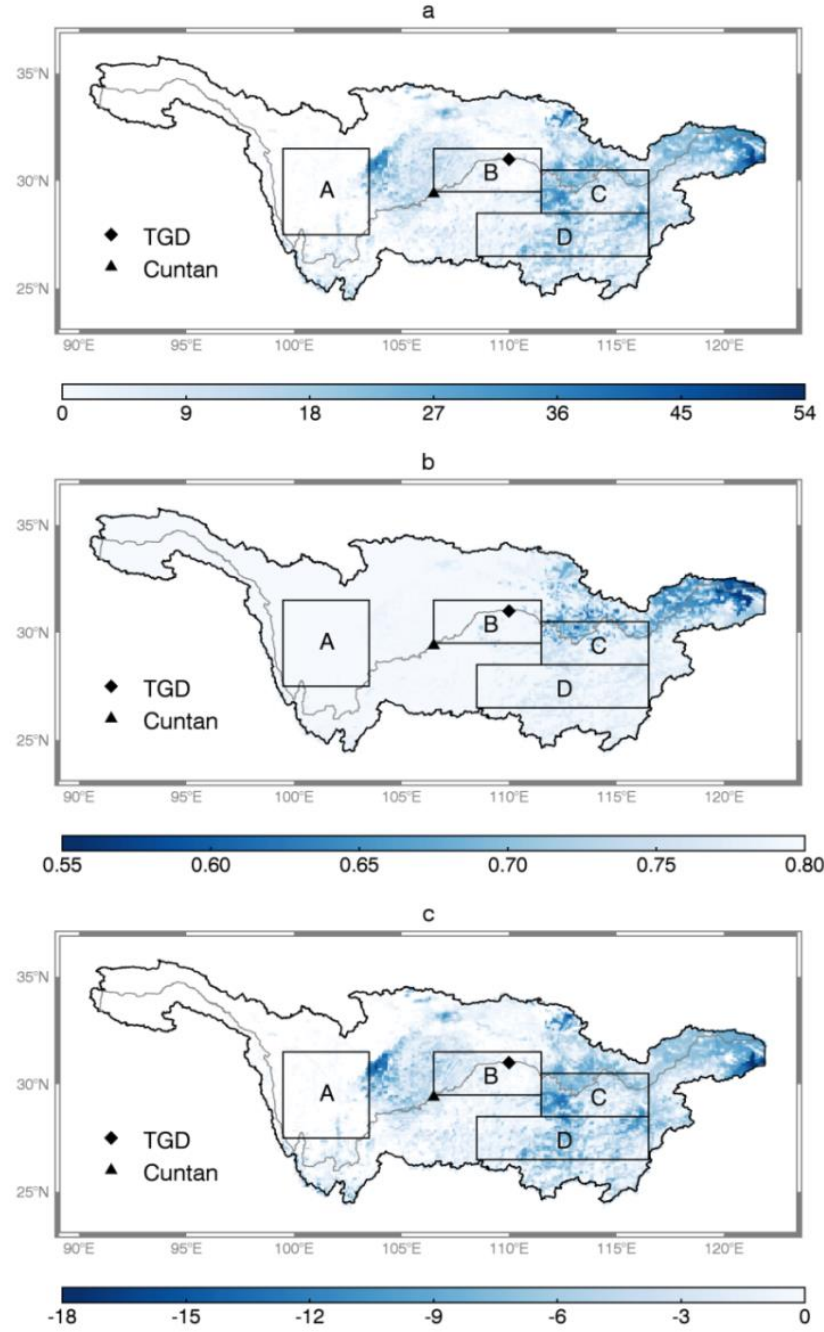

Figure 7.5. (a) Irrigated area, in $\mathrm{km}^{2}$, (b) Fraction of return flows $\mathrm{f}_{\mathrm{rgi}}$ from irrigation to groundwater, and (c) Yearly average net abstraction of groundwater $N A_{g}$, in $\mathrm{cm} \mathrm{yr}^{-1}$, for the period 2005-2010, with the spatial resolution of 5 minutes. Negative net abstraction of groundwater indicates groundwater storage gains. 
Table 7.1. Water supply, use, and consumption in the Yangtze River basin during the period 2005-2010 $\left(\times 10^{9} \mathrm{~m}^{3}\right)$

\begin{tabular}{|c|c|c|c|c|c|c|c|c|c|c|c|c|c|}
\hline & $\begin{array}{c}\text { Surface } \\
\text { water } \\
\text { withdra } \\
\text { wal }\end{array}$ & $\begin{array}{c}\text { Ground } \\
\text { water } \\
\text { withdra } \\
\text { wal }\end{array}$ & $\begin{array}{c}\text { Total } \\
\text { water } \\
\text { supply/ } \\
\text { use }\end{array}$ & $\mathrm{WU}_{\mathrm{i}}$ & $\mathrm{WU}_{\mathrm{m}}$ & $\mathrm{WU}_{\mathrm{d}}$ & $\mathrm{WU}_{\mathrm{e}}$ & $\mathrm{WU}_{\mathrm{gi}}$ & $\mathrm{WU}_{\mathrm{si}}$ & $\mathrm{CU}_{\mathrm{gi}}$ & $\mathrm{CU}_{\mathrm{si}}$ & $\begin{array}{c}\text { WU- } \\
\mathrm{CU}\end{array}$ & $\mathrm{NA}_{\mathrm{g}}$ \\
\hline 2005 & 175.6 & 7.9 & 184.2 & 94.3 & 64.6 & 23.2 & 2.2 & 4.1 & 89.9 & 2.8 & 34.0 & 57.1 & -36.9 \\
\hline 2006 & 179.7 & 8.3 & 188.4 & 94.3 & 67.9 & 23.8 & 2.5 & 4.1 & 90.0 & 2.9 & 34.0 & 57.2 & -36.6 \\
\hline 2007 & 185.3 & 8.1 & 194.0 & 93.3 & 72.9 & 24.6 & 3.2 & 3.9 & 89.1 & 2.7 & 33.7 & 56.6 & -36.4 \\
\hline 2008 & 186.2 & 8.3 & 195.2 & 94.8 & 71.8 & 25.1 & 3.5 & 4.0 & 90.5 & 2.8 & 34.2 & 57.5 & -36.8 \\
\hline 2009 & 187.9 & 8.5 & 197.0 & 97.0 & 72.0 & 26.0 & 2.0 & 4.2 & 92.5 & 2.9 & 35.0 & 58.8 & -37.6 \\
\hline $\begin{array}{r}2010 \\
\text { averag }\end{array}$ & 189.0 & 8.5 & 198.3 & 94.8 & 74.7 & 26.9 & 2.0 & 4.1 & 90.4 & 2.9 & 34.2 & 57.4 & -36.6 \\
\hline e & 184.0 & 8.3 & 192.9 & 94.8 & 70.6 & 24.9 & 2.6 & 4.1 & 90.9 & 2.8 & 34.2 & 57.4 & -36.8 \\
\hline
\end{tabular}

$W U_{g i}$ is the withdrawal water from groundwater used for irrigation; $W U_{s i}$ is the withdrawal water from surface water for irrigation; $W U_{i}$ is the withdrawal water from surface water and groundwater for irrigation; $W U_{m}$ is the withdrawal water from surface water and groundwater for manufacturing; $W U_{d}$ is the withdrawal water from surface water and groundwater for domestic; $W U_{e}$ is the withdrawal water from surface water and groundwater for environment; $C U_{g i}$ is the consumptive water use from groundwater for irrigation; $C U_{s i}$ is the consumptive water use from surface water for irrigation; WU-CU is the return flow from irrigation to surface water ultimately; $N A_{g}$ is the net abstraction of groundwater.

Figure 7.5a illustrates that Region $C$ and the river mouth are the most intensive irrigation areas of the Yangtze River basin, and Figure 7.5b shows that the lower reaches of the basin receive slightly smaller fractions of the return flows from irrigation to groundwater, due to the fact that the lower reaches are more artificially drained. Figure $7.5 \mathrm{c}$ exhibits the spatial pattern of yearly average net abstraction of groundwater $\left(N A_{g}\right)$, which was calculated from the values of irrigation areas, fractions of the return flows $\left(f_{r g i}\right)$, and water use and supply data released by the Ministry of Water Resources of China (Table 7.1). Great negative values in the middle and lower reaches of the basin indicate that large amounts of irrigated water recharged groundwater, especially in Region C. We further computed the spatially averaged time series of net abstraction of groundwater in Regions A, B, C, and D, respectively, as shown in Table 7.2. In the selected four $\mathrm{ROHs}$, Region $\mathrm{C}$ experienced the greatest groundwater gains, with a mean rate of $4.2 \mathrm{~cm} \mathrm{yr}^{-1}$. The groundwater also increased in Regions B and D during the study period, but at a lower rate than in Region C, namely 1.2 and $3.0 \mathrm{~cm} \mathrm{yr}^{-1}$, respectively. 
Table 7.2. The human-induced net groundwater gain $\left(-N A_{\mathrm{g}}\right)$ and groundwater recharge, calculated using the method of Döll et al. (2012) in Regions A, B, C, and D. It should be noted that, in order to facilitate a comparison of the alternative estimates, the increments in the estimated human-induced TWS based on GRACE and LSMs (Figure 7.4) are also shown here. Units: $\mathrm{cm} \mathrm{yr}^{-1}$.

\begin{tabular}{ccccc}
\hline & Region A & Region B & Region C & Region D \\
\hline$-N A_{\mathrm{g}}$ & 0.2 & 1.2 & 4.2 & 3.0 \\
Groundwater recharge & 0.3 & 1.5 & 5.2 & 3.6 \\
\hline GRACE-Noah-MP & -0.5 & 1.1 & 3.7 & 1.9 \\
GRACE-ERA-Interim/Land & -0.7 & 1.3 & 3.9 & 1.4 \\
\hline
\end{tabular}

\subsection{Discussion}

In this chapter, we designed a framework for detection and attribution of spatial TWS changes, and took the Yangtze River basin as an example to investigate the spatial effects of human-induced changes on TWS. The human-induced TWS variations in the Yangtze River basin have been preliminarily estimated from GRACE remotely sensed data and LSM simulations over the 8-year period studied. GRACE detected TWS variations were affected by both climate variability and human interference, whereas the LSMs we used in this study simulated only the natural part of TWS variations taking no account of human activities. Without consideration of the feedback between human water use and terrestrial water fluxes, the human-induced TWS variations can thus be inferred from GRACE data by isolating the LSM simulated TWS, given auxiliary information on the other components of TWS.

Two sets of LSM simulated TWS data were used in this study, one was simulated by Noah-MP and driven by the ITPCAS meteorological data, and the other used ERA-Interim/Land data. GRACE senses all phases of water stored above and below the surface of the Earth, and hence the GRACE-observed TWS variations include the combined contributions of SM, canopy water storage, snow water equivalent, ice, biomass, surface water and groundwater. The estimated TWS of both Noah-MP and ERA-Interim/Land, however, lacks certain components due to missing processes and storage parameters, although these differ for the two. More specifically, the Noah-MP estimated TWS only includes SM, canopy water storage, 
snow water equivalent and groundwater, whereas the estimated TWS from ERAInterim/Land is limited to SM and snow water equivalent. Ice and biomass account for a tiny percentage of TWS in the Yangtze River basin, and thus have negligible impacts on TWS variations (Yang et al., 2011). Compared to the Noah-MP simulated TWS, canopy water storage and groundwater components are missing in the ERA-Interim/Land produced TWS. However, canopy water storage in the Yangtze River basin is negligible in comparison with SM (Yang et al., 2011), thereby having little impact on TWS variations. Moreover, the ERA-Interim/Land model, HTESSEL, has a deep soil reservoir to mimic shallow groundwater storage variations taking into account a capillary connection between groundwater and evaporation to avoid warm biases. Consequently, the estimated TWS from ERAInterim/Land to some extent reflects the groundwater variations, although it does not explicitly model groundwater (Balsamo et al., 2009). Furthermore, missing or poor model representations of, for instance, snow, surface water bodies and groundwater, predominantly affect the amplitudes of seasonal variations in TWS rather than natural inter-annual trends (Swenson and Wahr, 2006; Swenson and Milly, 2006; Syed et al., 2008). This is due to the fact that the physical consistency (i.e. closure of the water and energy budgets) is maintained by constructing in LSMs, and hence the LSM simulated TWS can largely represent the natural variability of meteorological fields.

Since simulated TWS from the LSMs does not account for all the components of TWS (e.g. surface water storage component), which is contrary to GRACE-derived TWS, our proposed method to estimate the human impacts is recommended for catchments with negligible changes in those components. Moreover, the use of two models is supported by the current developments in LSMs, whereby ensemble models are employed to average bias (Yang et al., 2011). Although these models have their own limitations, and using only two models does not necessarily reduce the errors, the comparable simulations from two validated models may improve our confidence in the obtained natural variability of TWS.

As shown in Figure 7.2, both the trends in estimated human-induced TWS variations from GRACE-Noah-MP (Figure 7.2a) and from GRACE-ERAInterim/Land (Figure 7.2b) show very similar patterns to GRACE (Figure 7.2c), which illustrates that neither LSMs simulated TWS exhibits significant trends 
during the study period. This demonstrates that the estimated human-induced TWS trends were not mathematical artifacts caused by subtraction of large negative LSM simulated TWS trends from GRACE TWS trends, and indicates that TWS trends, mainly originated from the GRACE solutions, were very likely caused by anthropogenic modification to the hydrological cycle rather than natural climate variability.

The estimated human-induced TWS changes from GRACE and LSMs can be validated by quantifying the impacts of water uses on surface and groundwater. The Yangtze River basin involves intensive human activities, such as irrigation, and dam construction. The basin is rich in surface water with approximately $95 \%$ of the water supply originating from surface water, while the other $5 \%$ is from groundwater (Table 7.1). Water used for irrigation takes up a large amount of the water supply, and accounts for more than $50 \%$ (around $94.8 \times 10^{9} \mathrm{~m}^{3}$ ) of total water use. The irrigated water is partly consumed by ET and outflow to the surface water bodies, whereas the other part recharges groundwater. Approximately $57.4 \times 10^{9} \mathrm{~m}^{3}$ of water was available to return to surface and groundwater storage per year (Table 7.1), with $55 \% \sim 80 \%$ recharging groundwater (Figure $7.5 \mathrm{~b}$ ). Due to irrigation, the mean net groundwater gain was nearly $36.8 \times 10^{9} \mathrm{~m}^{3} \mathrm{yr}^{-1}$ (Table 7.1). As shown in Figure 7.5c, the middle and lower reaches of the basin experience large amounts of net groundwater gain due to irrigation. The spatial pattern of net abstraction/gain of groundwater matches the pattern of estimated human-induced TWS changes based on GRACE and LSMs well (Figure 7.2).

Four ROHs (Regions A, B, C, and D) within the study area were chosen based on TWS growth rates (Figure 7.2) for further examination and comparison. It should be noted that Region B is the hydraulic basin of the TGR, and that we selected this region to quantify the impacts of TGR impoundment on TWS changes. As shown in Figure 7.4, Region C experienced dramatic human-induced TWS increment at a mean rate of $3.7 \pm 0.8 \mathrm{~cm} \mathrm{yr}^{-1}$ estimated from GRACE and Noah-MP, and a rate of $3.9 \pm 1.1 \mathrm{~cm} \mathrm{yr}^{-1}$ estimated from GRACE and ERA-Interim/Land, respectively. Regions B and D also show positive human-induced TWS trends, although less than Region C, while Region A exhibits no significant trend. This is consistent with the yearly average net gain of groundwater, estimated based on the method of Döll et al. (2012) (Table 7.2): 0.2, 1.2, 4.2, and $3.0 \mathrm{~cm} \mathrm{yr}^{-1}$ added to groundwater storage 
in Regions A, B, C, and D, respectively. The quantification of the impact of water use on groundwater storages, based on the method of Döll et al. (2012), is fully independent of the human-induced estimates from GRACE and LSMs, thus the high consistency between these methods verifies the estimated values and patterns of human-induced TWS, and also indicates that groundwater recharge from intensive surface water irrigation is an important contributor to the TWS increases in Regions B, C, and D.
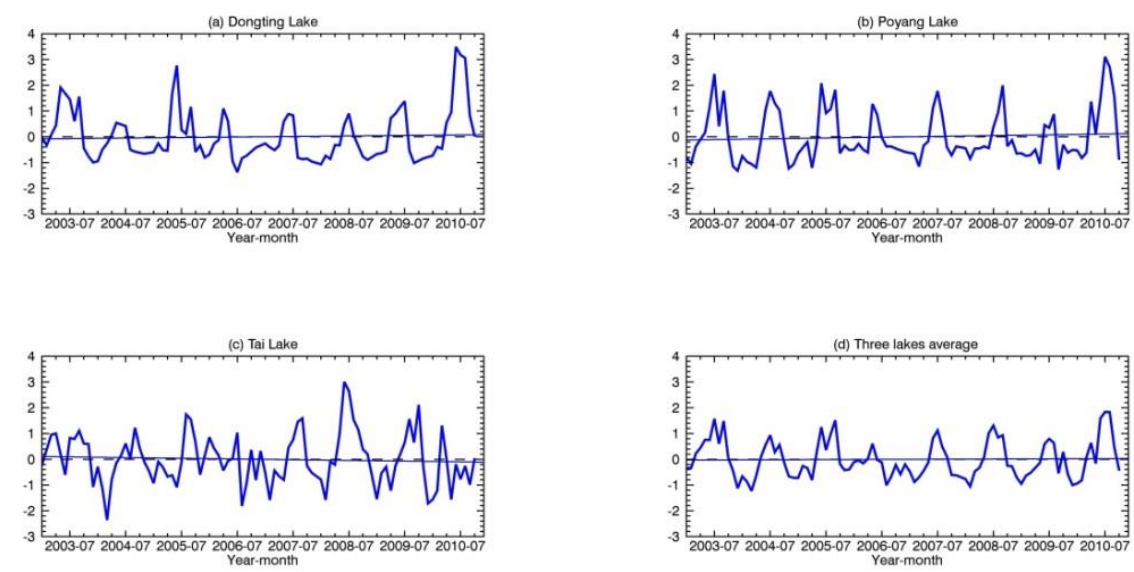

Figure 7.6. Monthly standardized anomalies of water level (blue curve) and trends (blue line), during the study period, for (a) Donging Lake, (b) Poyang Lake, (c) Tai Lake, and (d) the three lakes' average, respectively.

Surface water storage (e.g. lakes, rivers) merits consideration. As shown in Figure 5.7, the monthly discharge from the main hydrological gauging stations (Cuntan, Yichang, and Datong) and from model simulations on the Yangtze River mainstream are rather stationary except in 2006. Similarly, it can be clearly seen from Figure 7.6 that no trends are exhibited in water level variations for the three largest natural lakes (Dongting Lake, Poyang Lake, and Tai Lake), provided by HYDROWEB during the study period. In combination with the information that there was no rise in the water surface areas of Dongting Lake and Poyang Lake estimated from remotely sensed data (Hervé et al., 2011), we can infer that the lake volumes of Dongting Lake and Poyang Lake did not experience positive trends during the study period. It should be noted that these two lakes, Dongting Lake 
and Poyang Lake, are located in Region C (Figure 2.2). Therefore, rivers and natural lakes did not contribute to the GRACE-observed TWS trends in the Yangtze River basin, at least in Region C, during the study period.

Groundwater and surface water are physically connected by the hydrologic cycle, and their interactions depend on the physiographic and climatic setting of the landscape. Due to the fact that these interactions take many forms, and so far have not been well investigated in the Yangtze River basin, we cannot elaborate on the processes of groundwater and surface water interaction. However, it is reasonable that the surface-water bodies exhibited no trends while groundwater was continually increasing, because, first of all, the inter-annual changes in surfacewater bodies may not be sufficiently sensitive to changes in groundwater, as water that recharges groundwater originally comes from the surface water bodies. Second, the surface water bodies may gain water from inflow of groundwater in some stream reaches and lose water in other reaches, depending on the comparison between the altitude of the water table in the vicinity of streams and the altitude of the stream-water surface. Third, in some regions, the groundwater system may be disconnected from streams by an unsaturated zone, where changes in groundwater storage do not affect the flow of streams (Winter et al., 1998).

Groundwater flow is much slower than riverine flows, thus the part of irrigated water that recharges groundwater can accumulate underground. Therefore, the GRACE-derived TWS (Figure 7.2c) was continuously increasing. It should be noted that Figure 7.4 shows a decline in the GRACE-derived and human-induced TWS during 2006, which is inconsistent with the estimates based on the method of Döll et al. (2012). More specifically, the GRACE-derived TWS decreased by $3.7 \mathrm{~cm}$ on average in Region B and $6.7 \mathrm{~cm}$ in Region C in 2006 relative to the previous year 2005, whereas the estimated human-induced net groundwater gains were $1.2 \mathrm{~cm}$ in Region B and $4.2 \mathrm{~cm}$ in Region C (Table 7.2). This inconsistency can be explained by the 2006 extreme drought. As previously mentioned, an extreme drought occurred in 2006 in the Yangtze River basin. Dai et al. (2010) have shown that groundwater discharge along the middle and lower reaches plays an important role in the compensation of runoff and river level reduction in drought years. The total groundwater discharge was found to account for 31\% of the increased river discharge between Yichang and Datong in 2006. However, the net abstractions of 
groundwater estimated from the method of Döll et al. (2012) consider no such compensation. It is also interesting that the net groundwater gain is very high in the Yangtze delta (Figure 7.5c), which is not captured by GRACE data (Figure 7.2). This might be due to the fact that the Yangtze delta was formed by a series of Chenier shell ridges that gradually extended the deltaic plain seaward through the sedimentary deposition of the Yangtze River, and that groundwater flow rates are relatively high in these high permeability materials. The net gain of groundwater, thus, very likely flows away from the delta to neighboring regions.

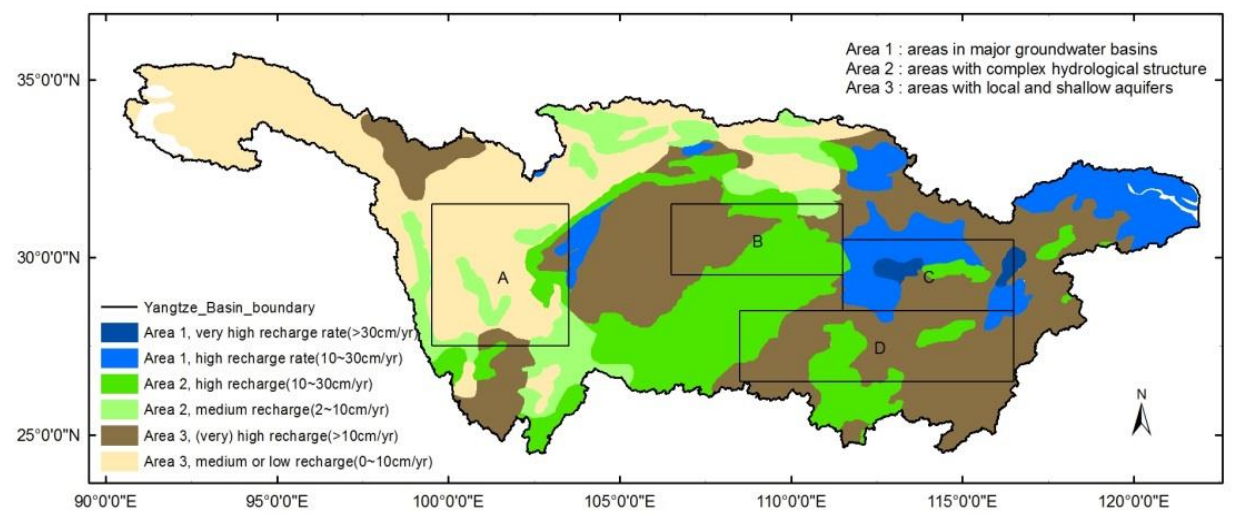

Figure 7.7. Groundwater resources map with aquifer systems in the Yangtze River basin (WHYMAP, 2008). Blue, green, and brown colors represent the areas in major groundwater basins (Area 1), with complex hydrological structure (Area 2), and with local and shallow aquifers (Area 3), respectively.

Light and dark colors represent the areas with relatively low and high recharge rate.

There are other factors that were not considered in the quantification of net groundwater recharge. First, the underlying soil and geology may partly explain the differences in groundwater variations between the four regions. As shown in Figure 7.7, Region C is largely underlain by a major groundwater basin, with a high available recharge rate of $10 \sim 30 \mathrm{~cm} \mathrm{yr}^{-1}$ or a very high recharge rate of more than $30 \mathrm{~cm} \mathrm{yr}^{-1}$. Regions B and D, located in areas with local and shallow aquifers or complex aquifers, also have a high recharge rate of $10 \sim 30 \mathrm{~cm} \mathrm{yr}^{-1}$. In contrast, Region A has a relatively low groundwater recharge rate of less than $10 \mathrm{~cm} \mathrm{yr}^{-1}$ (WHYMAP, 2008). The groundwater replenishment in Regions B, C and D, thus, occurs easily when there is a water surplus, particularly in Region C. Second, the decrease of NDVI (Figure 7.8) in Regions B, C and D indicates deterioration of the 
vegetation during the study period. The importance of vegetation in controlling water recharge in a natural system has been illustrated by previous studies (Wang et al., 2004; Scanlon et al., 2005, 2006), which show that changing land cover from vegetated to non-vegetated conditions increases groundwater recharge, as the decrease of vegetation cover reduces the ability of soil to retain water for plant use. This is consistent with the results that Regions B, C and D had a decrease in NDVI but an increase in groundwater storage. All of the above implies that the intensive surface water irrigation, leading to groundwater gains, forms a main contributor to the GRACE-derived TWS trends in the middle and lower reaches of the Yangtze River basin.

Water impoundment in reservoirs can cause a large mass redistribution. Wang et al. (2011) proved that the water storage changes in the TGR can be captured by GRACE, which explains $76 \%$ of the monthly variability in in-situ measurements. Thus, the GRACE-derived TWS trend in Region B, where the TGR is located, was also affected by the TGR operations. The impounded water volume of the TGR was 11.6, 5.6, and $6.1 \mathrm{~km}^{3}$ during the first filling stage in June 2003, the second filling stage in 2006, and the third filling stage in 2008, respectively. Due to the TGR impoundment, Region B experienced a TWS increment at a mean rate of $0.3 \mathrm{~cm} \mathrm{yr}^{-1}$ during the study period, as calculated by adding up the impounded water volumes of all three filling stages $\left(23.3 \mathrm{~km}^{3}\right)$ and dividing this figure by the area of Region B (about $1 \times 10^{5} \mathrm{~km}^{2}$ ) and the value of 8 (for the 8 -year period). This value of $0.3 \mathrm{~cm} \mathrm{yr}^{-1}$ is relatively small (24\%) compared to the net groundwater gain $\left(-N A_{g}\right)$ in Region $\mathrm{B}\left(1.2 \mathrm{~cm} \mathrm{yr}^{-1}\right)$. Considering both water impoundment in the TGR and net groundwater gain due to irrigation, the total annual TWS trend of Region B amounts to $1.5 \mathrm{~cm} \mathrm{yr}^{-1}$. This value is within the range of human-induced TWS changes estimated from GRACE-Noah-MP $\left(1.1 \pm 1.1 \mathrm{~cm} \mathrm{yr}^{-1}\right)$, and the estimate from GRACE-ERA-Interim/Land $\left(1.3 \pm 0.6 \mathrm{~cm} \mathrm{yr}^{-1}\right)$. The TGR water impoundment, thus, accounted for nearly $20 \%$ of the human-induced TWS changes. However, Region B, where the TGR is located, exhibits a less significant trend than Region C does. This indicates that water impoundment in reservoirs is an important contributor, but not the dominant one to the large-scale TWS changes in the Yangtze River basin. 

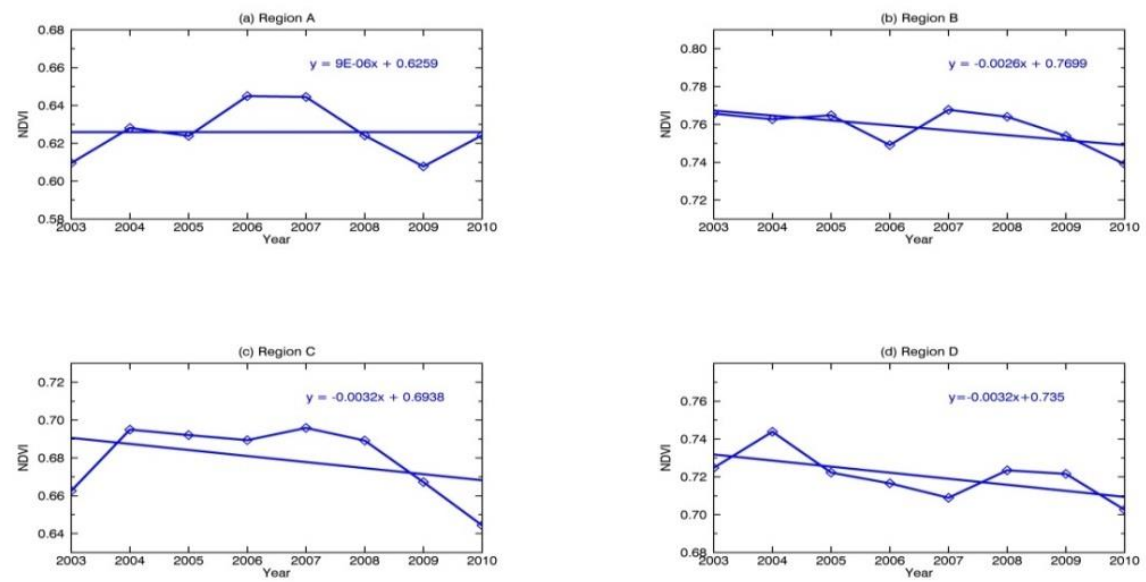

Figure 7.8. Spatial averaged annual NDVI changes (blue diamond curve) in summer and associated trends (blue line), during the study period, in Regions A, B, C and D.

The feedback between human water use and terrestrial water fluxes needs careful consideration. On the one hand, human water use is influenced by climate variation. When using equation (7.1) to estimate the human-induced TWS changes, it includes the uncertainties caused by the influences of climate variation on human water use. Climate variability may change, for instance, irrigation water demand, and consequently change water use and withdrawal. The stable climate during the study period, however, does not cause big changes in water use, and can largely reduce the uncertainties. As mentioned previously, the climate in the Yangtze River basin has been relatively stationary for the period 2003-2010. As a result, the changes in water use caused by climate variability are small, which can also be confirmed by the small changes in irrigation water use data, as listed in Table 7.1. Therefore, the associated discrepancy between the estimated humaninduced TWS changes and the actual values is small. On the other hand, climate variation is influenced by human water use. As previously mentioned, the Yangtze River basin, especially the middle and lower reaches, is dominated by the ET demand limited regime, where terrestrial water fluxes are mainly controlled by net radiation rather than SM. Hence, human water use such as irrigation causes changes in SM, but has little influence on climate variation. 


\subsection{Conclusions}

In this study, we proposed a framework for detection and attribution of spatial TWS changes. This framework integrates GRACE satellite detection and macroscale hydrological models, and gives water managers and researchers a useful tool to investigate the spatial human effects on TWS in various climate regimes. Moreover, this proposed framework provides valuable insights for regions where in situ data are inaccessible. For regions with some in situ data of, for instance, groundwater levels, it is recommended to use data assimilation, which can combine the virtues of in situ data and hydrological modeling, rather than solely use point measurements, to validate GRACE satellite estimates. Furthermore, the framework takes into account the feedback between human water use and terrestrial water fluxes, and stresses the importance of selecting a climate stationary period as the study period and, based on SM-climate interactions, of choosing a suitable hydrological model to validate the GRACE detection, which is very different from previous GRACE applications (e.g. Rodell et al., 2009; Feng et al., 2013; Voss et al., 2013).

This framework is illustrated by its application to the Yangtze River basin, due to the fact that GRACE data have rarely been applied in a water rich basin to estimate spatial effects of human-induced changes on TWS mainly caused by large-scale irrigation. For this basin, we conclude that the spatial pattern of TWS, especially in the middle and lower reaches, was changing during the period 2003-2010, and this change was occurring as a result of anthropogenic modification to the hydrological cycle rather than natural climate variability. Human activity such as intensive surface water irrigation and reservoir operation were increasing the TWS continuously in the middle and lower reaches, as was captured by subtracting LSM simulations from GRACE satellite data. This human-induced increase in TWS was mainly attributed to intensive surface water irrigation, which leads to groundwater gains. The TGR impoundment was also a noticeable cause for TWS change in the reservoir region and accounted for nearly $20 \%$ of the human-induced TWS increment. However, we should acknowledge the shortcomings, such as the absence of groundwater measurements and detailed information on reservoir construction and management in the case study. 
CHAPTER 8

Conclusions 



\section{Chapter 8 Conclusions}

Effects of climate variability and human activities on river basins have received increasing attentions, as extreme weather and climate events occur more frequently in some regions (IPCC, 2012). Terrestrial water storage (TWS) strongly influences water and energy fluxes, thereby playing a major role in the Earth's climate system (Famiglietti, 2004). A better understanding of TWS variability and its response to climate variability and human activity is thus important. However, mainly due to the fact that TWS is not routinely assessed like other hydrometeorological measurements, few studies have paid attention to TWS distribution in time and space.

With this background, the main objective of the dissertation is to investigate the space-time variability in TWS and its response to climate variability and human activity in the Yangtze River basin. In order to achieve this, the publicly available data assimilation products, remotely sensed observations, in situ data and modelling techniques are used. I first focus on obtaining reliable climate-related TWS estimates by evaluating publicly available data assimilation products and/or reconstructing the water budget from land surface models (LSMs) for the Yangtze River basin (Chapters 3-5). Based on this, Chapter 6 analyzes the climate-related TWS variations in space and time, and Chapter 7 investigates the spatial effects of human-induced changes in TWS in the Yangtze River basin.

\subsection{Climate-related TWS estimation}

Chapter 3 evaluates the TWS estimates from the ERA-Interim Reanalysis Data (ERA-Interim) and the Global Land Data Assimilation System with Noah LSM (GLDAS-Noah). The regional accuracies and reliabilities of the ERA-Interim and GLDAS-Noah datasets are assessed by comparing their spatially averaged time series of runoff for the upper Yangtze River with the observed discharge for the period 1979-2004, and the results show that the ERA-Interim modeled runoff fits the observed values better than the GLDAS-Noah modeled runoff does. 
Besides publicly available global data assimilation products, the TWS variations of the Yangtze River basin can also be estimated from LSM simulations. This simulated TWS may have higher accuracy than global data assimilation products, because of the improved atmospheric forcing data and more realistic representations of physical processes for the study area. Therefore, the ITPCAS atmospheric data, which have been proved to have higher accuracy in China than the GLDAS atmospheric data, are used to force the LSMs in Chapters 4 and 5 . Moreover, the new roughness lengths derived by Zheng et al. (2014) are implemented in the Noah LSM for the regional scale land surface modelling of alpine grasslands in the Yangtze River basin (Chapter 4), and the Noah LSM with multi-parameterization options (Noah-MP) is used in Chapter 5 to investigate to which extent this augmented Noah LSM can improve the simulation accuracy of water states and fluxes.

Chapter 4 assesses the effects of roughness length parameterizations on regional scale land surface modelling of alpine grasslands in the Yangtze River basin. The results show that the newly derived roughness lengths not only generally reduce the mean biases of daytime land surface temperature $\left(T_{s f}\right)$ simulation of the Noah LSM, but also largely improve the simulation performance of water budget components (e.g. runoff). On the other hand, the Noah LSM with the updated roughness lengths still largely underestimates the daytime $T_{s f c}$ during the winter period and the monthly runoff, which can be attributed to the imperfect roughness length schemes, unrealistic representations of other processes, and the inaccuracies in the forcing data. Furthermore, it is recommended to use the roughness length scheme proposed by Chen and Zhang (2009) (C09) with the updated roughness length for momentum transfer $\left(z_{0 m}\right)$ for the Noah land surface modelling of alpine grasslands, particularly in dry conditions.

Alternative parameterizations within the same framework are compared in Chapter 5, in order to investigate which role of a specific process in controlling water fluxes and states in the Yangtze River basin. The results show that Noah-MP can significantly reduce the mean bias error (MBE) and improve the model efficiency of monthly runoff simulation compared to the Noah LSM. This improvement can be mainly attributed to the improved physics of supercooled 
liquid water, frozen soil permeability and the lower boundary condition of soil moisture.

\subsection{TWS variations caused by climate variability}

Chapters 3, 4, and 5 show that the ERA-Interim datasets perform better in the Yangtze River basin than the GLDAS-Noah, the updated Noah LSM, and the Noah-MP in terms of runoff simulation. Therefore, Chapter 6 analyzes the spatial and temporal variations of the TWS in the Yangtze River basin during the period 1979 to 2010 based on ERA-Interim datasets. The results show that the Yangtze River basin is drying up, especially since the year 1998. The TWS variation is strongly correlated to the precipitation variation derived from ERA-Interim, the GPCC and PREC/L data, especially during the wet season and in the middle and lower reaches, suggesting the TWS variation is mainly controlled by precipitation. Moreover, in the middle and lower Yangtze reaches, the TWS behavior changed abruptly and started to decrease in 2004. The past 6-year period (2005-2010) was the driest period in terms of TWS in the Yangtze River basin since 1979. This is mainly the result of a dramatic decrease in precipitation.

\subsection{Human-induced changes in TWS}

Chapter 7 proposed a conceptual framework for detection and attribution of human-induced changes in TWS. This framework integrates GRACE satellite detection and macro-scale hydrological models, and gives water managers and researchers a useful tool to investigate the spatial human effects on TWS in various climate regimes. Particularly, it provides valuable insights for regions where ground-based measurements are inaccessible, and takes into account the feedback between human water use and terrestrial water fluxes.

This framework is illustrated by its application to the Yangtze River basin. For this basin, the spatial pattern of TWS, especially in the middle and lower reaches, was changing during the period 2003-2010, and this change was occurring as a result of anthropogenic modification to the hydrological cycle rather than natural climate variability. Human activities such as intensive surface water irrigation and reservoir operation were increasing the TWS continuously in the middle and lower 
reaches. This human-induced increase in TWS was mainly attributed to intensive surface water irrigation, which leads to groundwater gains. The three gorges reservoir (TGR) impoundment was also a noticeable cause for TWS changes in the area where it is located, which accounted for nearly $20 \%$ of the human-induced TWS increment.

\subsection{General conclusions}

This dissertation has demonstrated the value of integration of earth observations with modeling techniques in understanding the effects of climate variability and human activities on river basins. It also highlights the importance of a spatial perspective in water resources investigation. Although there is no substitute for ground-based observational data, emerging advances in hydrologic remote sensing and hydrological models, combined with better understanding of the hydrologic cycle, allow us to gradually break the limit of in situ data unavailability, and construct a holistic picture of changing water availability for a particular region or across the globe. This $\mathrm{PhD}$ research reflects that science-informed perspective.

\subsection{Suggestions for future research}

Model simulation plays a key role in quantifying the effects of climate variability and human activity on water resources, particularly from a spatial perspective. In this dissertation, the natural TWS variations are mainly estimated from LSMs. Despite good progress made in land surface modelling in recent decades, it is still a challenge to achieve reliable simulations in certain regions (e.g. the Tibetan Plateau) or under certain conditions (e.g. dry conditions). Therefore, it is imperative to better understand land surface processes and further improve model physical parameterizations. In order to accomplish this, in situ measurements are crucial.

Besides, a more inclusive model system which can explicitly represent the effects of human activity on water resources is necessary. With such a model system, human influence on water resources can also be quantified through comparison between the simulated situations with and without human intervention within the same framework, besides comparing the simulated naturalized situation of the 
hydrological system with the observation (the real situation). This will offer great opportunities for regions where observations are unavailable. 


\section{Bibliography}

Alcamo, J., P. Döll, T. Henrichs, F. Kaspar, B. Lehner, T. Rösch, and S. Siebert, 2003a: Development and testing of the WaterGAP 2 global model of water use and availability. Hydrological Sciences Journal, 48(3), 317-337.

Alcamo, J., P. Döll, T. Henrichs, F. Kaspar, B. Lehner, T. Rösch, and S. Siebert, 2003b: Global estimates of water withdrawals and availability under current and future "business-as-usual" conditions. Hydrological Sciences Journal, 48(3), 339-348.

Balsamo, G., A. Beljaars, K. Scipal, P. Viterbo, B. van den Hurk, M. Hirschi, and A. K. Betts, 2009: A Revised Hydrology for the ECMWF Model: Verification from Field Site to Terrestrial Water Storage and Impact in the Integrated Forecast System. Journal of Hydrometeorology, 10, 623643.

Balsamo, G., et al., 2015: ERA-Interim/Land: a global land surface reanalysis data set. Hydrol. Earth Syst. Sci., 19(1), 389-407.

Ball, J. T., I. E. Woodrow, and J. A. Berry, 1987: A model predicting stomatal conductance and its contribution to the control of photosynthesis under different environmental conditions, in Process in Photosynthesis Research, vol. 1, edited by J. Biggins, pp. 221-234, Martinus Nijhoff, Dordrecht, Netherlands.

Barco, J., T. S. Hogue, M. Girotto, D. R. Kendall, and M. Putti, 2010: Climate signal propagation in southern California aquifers. Water Resources Research, 46, W00F05, doi:10.1029/2009WR008376.

Berrisford, P., 2011: The ERA-Interim Archive: Version 2.0. ECMWF.

Bonan, G. B., 1996: A land surface model (LSM version 1.0) for ecological, hydrological, and atmospheric studies: Technical description and user's guide, NCAR tech. Note NCAR/TN-417+STR, 150 pp., Natl. Cent. for Atmos, Res., Boulder, Colo.

Brunsell, N. A., D. B. Mechem, and M. C. Anderson, 2011: Surface heterogeneity impacts on boundary layer dynamics via energybalance partitioning, Atmos. Chem. Phys., 11, 3403-3416. 
Brutsaert, W., 1982: Evaporation into the Atmosphere: Theory, History and Applications. Springer, 319pp.

Brutsaert, W., 1998: Land-surface water vapor and sensible heat flux: Spatial variability, homogeneity, and measurement scales. Water Resources Research, 34, 2433-2442.

Burn, D. H., and M. A. Hag Elnur, 2002: Detection of hydrologic trends and variability. Journal of Hydrology, 255, 107-122.

Cai, X., Z.-L. Yang, C. H. David, G.-Y. Niu, and M. Rodell, 2014: Hydrological evaluation of the Noah-MP land surface model for the Mississippi River Basin. Journal of Geophysical Research: Atmospheres, 119, 2013JD020792.

Campbell, G. S., 1974: A simple method for determining unsaturated conductivity from moisture retention data. Soil Science, 117 (6). pp. 311-314.

Chen, F., Z. Janjić, and K. Mitchell, 1997: Impact of Atmospheric Surfacelayer Parameterizations in the new Land-surface Scheme of the NCEP Mesoscale Eta Model. Boundary-Layer Meteorology, 85, 391-421.

Chen, J. L., M. Rodell, C. R. Wilson, and J. S. Famiglietti, 2005: Low degree spherical harmonic influences on Gravity Recovery and Climate Experiment (GRACE) water storage estimates. Geophysical Research Letters, 32, L14405.

Chen, J. L., C. R. Wilson, B. D. Tapley, Z. L. Yang, and G. Y. Niu, 2009: 2005 drought event in the Amazon River basin as measured by GRACE and estimated by climate models, J. Geophys. Res., 114, B05404, doi: $10.1029 / 2008$ jb006056.

Chen, X., Z. Su, Y. Ma, S. Liu, Q. Yu, and Z. Xu, 2014: Development of a 10-year (2001-2010) $0.1^{\circ}$ data set of land-surface energy balance for mainland China. Atmos. Chem. Phys., 14, 13097-13117.

Chen, Y., K. Yang, D. Zhou, J. Qin, and X. Guo, 2010: Improving the Noah Land Surface Model in Arid Regions with an Appropriate Parameterization of the Thermal Roughness Length. Journal of Hydrometeorology, 11, 995-1006. 
Chen, F., and Y. Zhang, 2009: On the coupling strength between the land surface and the atmosphere: From viewpoint of surface exchange coefficients. Geophysical Research Letters, 36, L10404.

Chen, Y., K. Yang, J. He, J. Qin, J. Shi, J. Du, and Q. He, 2011: Improving land surface temperature modeling for dry land of China. Journal of Geophysical Research: Atmospheres, 116, D20104.

Collatz, G. J., J. T. Ball, C. Grivet, and J. A. Berry, 1991: Physiological and environmental regulation of stomatal conductance, photosynthesis and transpiration: a model that includes a laminar boundary layer. Agricultural and Forest Meteorology, 54(2-4), 107-136.

Collatz, G. J., M. Ribascarbo, and J. A. Berry, 1992: A coupled photosynthesis-stomatal conductance model for leaves of $\mathrm{C} 4$ plants. Aust. J. Plant Physiol., 19, 519-538, doi: 10.1071/PP9920519.

Crawford, T. M., and C. E. Duchon, 1999: An Improved Parameterization for Estimating Effective Atmospheric Emissivity for Use in Calculating Daytime Downwelling Longwave Radiation. Journal of Applied Meteorology, 38, 474-480.

Crétaux, J. F., et al., 2011: SOLS: A lake database to monitor in the Near Real Time water level and storage variations from remote sensing data. Advances in Space Research, 47(9), 1497-1507.

Dai, Z., J. Du, J. Li, W. Li, and J. Chen, 2008: Runoff characteristics of the Changjiang River during 2006: Effect of extreme drought and the impounding of the Three Gorges Dam. Geophysical Research Letters, 35(7), L07406.

Dai, Z.-j., J.-z. Du, A. Chu, J.-f. Li, J.-y. Chen, and X.-I. Zhang, 2010: Groundwater discharge to the Changjiang River, China, during the drought season of 2006: effects of the extreme drought and the impoundment of the Three Gorges Dam. Hydrogeol J, 18, 359-369.

Dee, D. P., et al., 2011: The ERA-Interim reanalysis: configuration and performance of the data assimilation system, Quarterly Journal of the Royal Meteorological Society, 137 (656), 553-597.

Ding, Y. H., 1992: Summer monsoon rainfalls in China, J. Meteor. Soc. Jpn., 70, 373-396. 
Ding, Y. H., and J. C. L. Chan, 2005: The East Asian summer monsoon: an overview. Meteorol. Atmos. Phys., 89, 117-142.

Ding, Y., Z. Wang, and Y. Sun, 2008: Inter-decadal variation of the summer precipitation in East China and its association with decreasing Asian summer monsoon. Part I: Observed evidences. International Journal of Climatology, 28, 1139-1161.

Ding, Y., Y. Sun, Z. Wang, Y. Zhu, and Y. Song, 2009: Inter-decadal variation of the summer precipitation in China and its association with decreasing Asian summer monsoon Part II: Possible causes. International Journal of Climatology, 29, 1926-1944.

Dirmeyer, P. A., 2011: The terrestrial segment of soil moisture-climate coupling. Geophysical Research Letters, 38, L16702.

Dirmeyer, P. A., R. D. Koster, and Z. Guo, 2006: Do Global Models Properly Represent the Feedback between Land and Atmosphere? Journal of Hydrometeorology, 7, 1177-1198.

Dorigo, W. A., K. Scipal, R. M. Parinussa, Y. Y. Liu, W. Wagner, R. A. M. de Jeu, and V. Naeimi, 2010: Error characterisation of global active and passive microwave soil moisture datasets. Hydrol. Earth Syst. Sci., 14, 2605-2616.

Dorigo, W. A., and Coauthors, 2011: The International Soil Moisture Network: a data hosting facility for global in situ soil moisture measurements. Hydrol. Earth Syst. Sci., 15, 1675-1698.

Dunne, T., 1978: Field studies of hillslope flow processes, in: Hillslope Hydrology, edited by: Kirkby, M. J., Wiley-Interscience, New York, 227293, 1978.

Döll, P., K. Fiedler, and J. Zhang, 2009: Global-scale analysis of river flow alterations due to water withdrawals and reservoirs. Hydrol. Earth Syst. Sci., 13(12), 2413-2432.

Döll, P., H. Hoffmann-Dobrev, F. T. Portmann, S. Siebert, A. Eicker, M. Rodell, G. Strassberg, and B. R. Scanlon, 2012: Impact of water 
withdrawals from groundwater and surface water on continental water storage variations. Journal of Geodynamics, 59-60(0), 143-156.

Entekhabi, D., and Coauthors, 2010: The Soil Moisture Active Passive (SMAP) Mission. Proceedings of the IEEE, 98, 704-716.

Famiglietti, J. S., 2004: Remote Sensing of TerrestrialWater Storage, Soil Moisture and Surface Waters, in: The State of the Planet: Frontiers and Challenges in Geophysics, Geophysical Monograph Series, 150, edited by: Sparks, R. S. J. and Hawkesworth, C. J., AGU, Washington, D. C., USA, 197-207.

Famiglietti, J. S., D. Ryu, A. A. Berg, M. Rodell, and T. J. Jackson, 2008: Field observations of soil moisture variability across scales. Water Resources Research, 44, W01423, doi:10.1029/2006WR005804.

Famiglietti, J. S., M. Lo, S. L. Ho, J. Bethune, K. J. Anderson, T. H. Syed, S. C. Swenson, C. R. de Linage, and M. Rodell, 2011: Satellites measure recent rates of groundwater depletion in California's Central Valley. Geophysical Research Letters, 38(3), L03403.

Feick, S., S. Siebert, and P. Döll, 2005: A Digital Global Map of Artificially Drained Agricultural Areas. Frankfurt Hydrology Paper 04. Institute of Physical Geography, University of Frankfurt, Frankfurt am Main, 57 pp.

Feng, W., M. Zhong, J.-M. Lemoine, R. Biancale, H.-T. Hsu, and J. Xia, 2013: Evaluation of groundwater depletion in North China using the Gravity Recovery and Climate Experiment (GRACE) data and ground-based measurements. Water Resources Research, 49(4), 2110-2118.

Fu, C. B., and G. Wen, 2002: Several issues on aridification in the northern China (in Chinese). Climatic and Environmental Research, 7, 22-29.

Fuchs, M., G. S. Campbell, and R. I. Papendick, 1978: An analysis of sensible and latent heat flow in a partially frozen unsaturated soil. Soil Sci. Soc. Amer. J., 42, 379-385.

Garratt, J. R., 1994: The Atmospheric Boundary Layer. Cambridge University Press, 336 pp. 
Giorgi, F., J. W. Hurrell, M. R. Marinucci, and M. Beniston, 1997: Elevation Dependency of the Surface Climate Change Signal: A Model Study. Journal of Climate, 10, 288-296.

Gulden, L. E., E. Rosero, Z.-L. Yang, M. Rodell, C. S. Jackson, G.-Y. Niu, P. J.-F. Yeh, and J. Famiglietti, 2007: Improving land-surface model hydrology: Is an explicit aquifer model better than a deeper soil profile? Geophysical Research Letters, 34, L09402.

Guo, H., Q. Hu, Q. Zhang, and S. Feng, 2012: Effects of the Three Gorges Dam on Yangtze River flow and river interaction with Poyang Lake, China: 2003-2008. Journal of Hydrology, 416-417(0), 19-27.

Hanasaki, N., S. Kanae, T. Oki, K. Masuda, K. Motoya, N. Shirakawa, Y. Shen, and K. Tanaka, 2008a: An integrated model for the assessment of global water resources - Part 1: Model description and input meteorological forcing. Hydrol. Earth Syst. Sci., 12(4), 1007-1025.

Hanasaki, N., S. Kanae, T. Oki, K. Masuda, K. Motoya, N. Shirakawa, Y. Shen, and K. Tanaka, 2008b: An integrated model for the assessment of global water resources - Part 2: Applications and assessments. Hydrol. Earth Syst. Sci., 12(4), 1027-1037.

Harvey, J. and L. Tang, 2007: Analysis of the Global Precipitation Climatology Project Data for Four Global River Basins, GPCPReport, GPCP, University of Maryland.

He, J., 2010: Development of surface meteorological dataset of China with high temporal and spatial resolution, M.S.thesis, Inst. of Tibetan Plateau Res., Chin. Acad. of Sci., Beijing, China.

Hervé, Y., et al., 2011: Nine years of water resources monitoring over the middle reaches of the Yangtze River, with ENVISAT, MODIS, Beijing-1 time series, Altimetric data and field measurements. Lakes \& Reservoirs: Research \& Management, 16(3), 231-247.

Hogue, T. S., L. Bastidas, H. Gupta, S. Sorooshian, K. Mitchell, and W. Emmerich, 2005: Evaluation and Transferability of the Noah Land Surface Model in Semiarid Environments. Journal of Hydrometeorology, 6, 68-84. 
Horton, R. E., 1933: The role of infiltration in the hydrologic cycle, Trans. Am. Geophys. Union, 14, 446-460.

IPCC, 2001: Climate Change 2001: The Scientific Basis. Contribution of Working Group I to the Third Assessment Report of the Intergovernmental Panel on Climate Change, edited by: Houghton, J. T., Ding, Y., Griggs, D. J., Noguer, M., van der Linden, P. J., Dai, X., Maskell, K., and Johnson, C. A., Cambridge University Press, Cambridge, United Kingdom and New York, NY, USA, 881 pp.

IPCC, 2012: Managing the Risks of Extreme Events and Disasters to Advance Climate Change Adaptation. A Special Report of Working Groups I and II of the Intergovernmental Panel on Climate Change [Field, C.B., V. Barros, T.F. Stocker, D. Qin, D.J. Dokken, K.L. Ebi, M.D. Mastrandrea, K.J. Mach, G.-K. Plattner, S.K. Allen, M. Tignor, and P.M. Midgley (eds.)]. Cambridge University Press, Cambridge, UK, and New York, NY, USA, $582 \mathrm{pp}$.

Huffman, G. J., and Coauthors, 2007: The TRMM Multisatellite Precipitation Analysis (TMPA): Quasi-Global, Multiyear, Combined-Sensor Precipitation Estimates at Fine Scales. Journal of Hydrometeorology, 8, 38-55.

Immerzeel, W. W., L. P. H. van Beek, and M. F. P. Bierkens, 2010: Climate Change Will Affect the Asian Water Towers. Science, 328, 1382-1385.

Jordan, R., 1991: A One-dimensional Temperature Model for a Snow Cover, Spec. Rep. 91-16, Cold Reg. Res. and Eng. Lab., U.S. Army Corps of Eng., Hanover, N. H.

Jung, M., et al., 2010: Recent decline in the global land evapotranspiration trend due to limited moisture supply. Nature, 467(7318), 951-954.

Kendall, M. G., 1975: Rank Correlation Measures, Charles Griffin, London.

Koike, T., 2004: The Coordinated Enhanced Observing Period: An initial step for integrated global water cycle observation. WMO Bull., 53, 115-121.

Koren, V., J. Schaake, K. Mitchell, Q. Y. Duan, F. Chen, and J. M. Baker, 1999: A parameterization of snowpack and frozen ground intended for 
NCEP weather and climate models. Journal of Geophysical Research: Atmospheres, 104, 19569-19585.

Kummu, M., P. J. Ward, H. de Moel, and O. Varis, 2010: Is physical water scarcity a new phenomenon? Global assessment of water shortage over the last two millennia, Environ. Res. Lett., 5(3), 034006.

Landerer, F. W., and S. C. Swenson, 2012: Accuracy of scaled GRACE terrestrial water storage estimates. Water Resources Research, 48, w04531.

LeMone, M. A., M. Tewari, F. Chen, J. G. Alfieri, and D. Niyogi, 2008: Evaluation of the Noah Land Surface Model Using Data from a FairWeather IHOP 2002 Day with Heterogeneous Surface Fluxes. Monthly Weather Review, 136, 4915-4941.

Li, C., Z. Yang, and X. Wang, 2004: Trends of Annual Natural Runoff in the Yellow River Basin. Water International, 29, 447-454.

Li, L.-J., and Coauthors, 2007: Assessing the impact of climate variability and human activities on streamflow from the Wuding River basin in China. Hydrological Processes, 21, 3485-3491.

Li, Y. H., H. M. Xu, and D. Liu, 2009: Features of the extremely severe drought in the east of South-west China and anomalies of atmospheric circulation in summer 2006, Acta Meterol. Sin., 67, 122-132.

Liu, Y., E. X. Zhao, G. F. Peng, and S. Q. Yang, 2007: Severe drought in the early summer of 2005 in Yunnan and middle-high latitudes circulation, Arid. Meteor., 25, 32-37. (In Chinese)

Liu, Y. Y., and Coauthors, 2011: Developing an improved soil moisture dataset by blending passive and active microwave satellite-based retrievals. Hydrol. Earth Syst. Sci., 15, 425-436.

Lo, M.-H., and J. S. Famiglietti, 2013: Irrigation in California's Central Valley strengthens the southwestern U.S. water cycle. Geophys. Res. Lett., 40, 301-306, doi:10.1002/grl.50108.

Long, H. L., G. K. Heilig, J. Wang, X. B. Li, M. Luo, X. Q. Wu, and M. Zhang, 2006: Land use and soil erosion in the upper reaches of the Yangtze 
River: some socio-economic considerations on China's Grain-for-Green Programme. Land Degradation \& Development, 17, 589-603.

Lorenzo-Lacruz, J., S. M. Vicente-Serrano, J. I. López-Moreno, S. Beguería, J. M. García-Ruiz, and J. M. Cuadrat, 2010: The impact of droughts and water management on various hydrological systems in the headwaters of the Tagus River (central Spain). Journal of Hydrology, 386(1-4), 1326.

Ma, Y., M. Menenti, R. Feddes, and J. Wang, 2008: Analysis of the land surface heterogeneity and its impact on atmospheric variables and the aerodynamic and thermodynamic roughness lengths. Journal of Geophysical Research: Atmospheres, 113, D08113.

Ma, Y., O. Tsukamoto, J. Wang, H. Ishikawa, and I. Tamagawa, 2002: Analysis of aerodynamic and thermodynamic parameters on the grassy marshland surface of Tibetan Plateau. Prog. Nat. Sci., 12, 36-40.

Ma, Z., and C. Fu, 2006: Some evidence of drying trend over northern China from 1951 to 2004. CHINESE SCI BULL, 51, 2913-2925.

Mahrt, L., and M. Ek, 1984: The Influence of Atmospheric Stability on Potential Evaporation. Journal of Climate and Applied Meteorology, 23, 222-234.

Mahrt, L., and H. Pan, 1984: A two-layer model of soil hydrology. BoundaryLayer Meteorology, 29, 1-20.

Mair, A., and A. Fares, 2010: Influence of groundwater pumping and rainfall spatio-temporal variation on streamflow. Journal of Hydrology, 393(34), 287-308.

Malik, M. J., R. van der Velde, Z. Vekerdy, and Z. Su, 2014: Improving modeled snow albedo estimates during the spring melt season. Journal of Geophysical Research: Atmospheres, 119, 7311-7331.

Mann, H. B., 1945: Nonparametric Tests Against Trend, Econometrica, 13, 245-259.

Martano, P., 2000: Estimation of surface roughness length and displacement height from single-level sonic anemometer data. J. Appl. Meteor., 39, 708-715. 
Miller, N. L., J. Jin, and C.-F. Tsang, 2005: Local climate sensitivity of the Three Gorges Dam, Geophys. Res. Lett., 32, L16704, doi: $10.1029 / 2005 \mathrm{gl} 022821$.

Moraes, J. M., G. Q. Pellegrino, M. V. Ballester, L. A. Martinelli, R. L. Victoria, and A. V. Krusche, 1998: Trends in Hydrological Parameters of a Southern BrazilianWatershed and its Relation to Human Induced Changes, Water Resour. Manage., 12, 295-311, doi:10.1023/a:1008048212420.

Moriasi, D. N., J. G. Arnold, M. W. Van Liew, R. L. Bingner, R. D. Harmel, and T. L. Veith, 2007: Model evaluation guidelines for systematic quantification of accuracy in watershed simulations. Trans. ASABE, 50, 885-900.

Morrison, F. A., 2014: Obtaining Uncertainty Measures on Slope and Intercept of a Least Squares Fit with Excel's LINEST. Michigan Technological University, Houghton

Nash, J. E., and J. V. Sutcliffe, 1970: River flow forecasting through conceptual models part I - A discussion of principles. Journal of Hydrology, 10, 282-290.

Niu, G.-Y., and Z.-L. Yang, 2004: Effects of vegetation canopy processes on snow surface energy and mass balances. Journal of Geophysical Research: Atmospheres, 109(D23), D23111.

Niu, G.-Y., and Z.-L. Yang, 2006: Effects of Frozen Soil on Snowmelt Runoff and Soil Water Storage at a Continental Scale. Journal of Hydrometeorology, 7(5), 937-952.

Niu, G.-Y., Z.-L. Yang, R. E. Dickinson, and L. E. Gulden, 2005: A simple TOPMODEL-based runoff parameterization (SIMTOP) for use in global climate models. Journal of Geophysical Research: Atmospheres, 110, D21106.

Niu, G.-Y., Z.-L. Yang, R. E. Dickinson, L. E. Gulden, and H. Su, 2007: Development of a simple groundwater model for use in climate models and evaluation with Gravity Recovery and Climate Experiment data. Journal of Geophysical Research: Atmospheres, 112 (D7), D07103. 
Niu, G.-Y., and Coauthors, 2011: The community Noah land surface model with multiparameterization options (Noah-MP): 1. Model description and evaluation with local-scale measurements. Journal of Geophysical Research: Atmospheres, 116, D12109.

Piao, S., and Coauthors, 2010: The impacts of climate change on water resources and agriculture in China, Nature, 467, 43-51.

Pokhrel, Y., N. Hanasaki, S. Koirala, J. Cho, P. J. F. Yeh, H. Kim, S. Kanae, and T. Oki, 2011: Incorporating Anthropogenic Water Regulation Modules into a Land Surface Model. Journal of Hydrometeorology, 13(1), 255-269.

Qian, W., H. S. Kang, and D. K. Lee, 2002: Distribution of seasonal rainfall in the East Asian monsoon region, Theor. Appl. Climatol., 73, 151-168, doi: 10.1007/s00704-002-0679-3.

Rodell, M., J. S. Famiglietti, J. Chen, S. I. Seneviratne, P. Viterbo, S. Holl, and C. R. Wilson, 2004: Basin scale estimates of evapotranspiration using GRACE and other observations. Geophysical Research Letters, 31, L20504.

Rodell, M., I. Velicogna, and J. S. Famiglietti, 2009: Satellite-based estimates of groundwater depletion in India. Nature, 460(7258), 9991002.

Rui, H, 2011: README Document for Global Land Data Assimilation System Version 1 (GLDAS-1) Products. Goddard Earth Sciences Data and Information Services Center, NASA, USA.

Ryu, Y., D. D. Baldocchi, S. Ma, and T. Hehn, 2008: Interannual variability of evapotranspiration and energy exchange over an annual grassland in California, J. Geophys. Res., 113, D09104, doi:10.1029/2007JD009263.

Salama, M. S., R. Van der Velde, L. Zhong, Y. Ma, M. Ofwono, and Z. Su, 2012: Decadal variations of land surface temperature anomalies observed over the Tibetan Plateau by the Special Sensor Microwave Imager (SSM/I) from 1987 to 2008. Climatic Change, 114, 769-781.

Savenije, H. H. G., A. Y. Hoekstra, and P. van der Zaag, 2014: Evolving water science in the Anthropocene. Hydrol. Earth Syst. Sci., 18(1), 319-332. 
Scanlon, B. R., R. C. Reedy, D. A. Stonestrom, D. E. Prudic, and K. F. Dennehy, 2005: Impact of land use and land cover change on groundwater recharge and quality in the southwestern US. Global Change Biology, 11(10), 1577-1593.

Scanlon, B. R., K. E. Keese, A. L. Flint, L. E. Flint, C. B. Gaye, W. M. Edmunds, and I. Simmers, 2006: Global synthesis of groundwater recharge in semiarid and arid regions. Hydrological Processes, 20(15), 3335-3370.

Schaake, J. C., V. I. Koren, Q.-Y. Duan, K. Mitchell, and F. Chen, 1996: Simple water balance model for estimating runoff at different spatial and temporal scales. Journal of Geophysical Research: Atmospheres, 101, 7461-7475.

Schneider, U., A. Becker, A. Meyer-Christoffer, M. Ziese, and B. Rudolf, 2011: Global Precipitation Analysis Products of the GPCC, Global Precipitation Climatology Centre (GPCC), Deutscher Wetterdienst, Offenbach a. M., Germany, December.

Scipal, K., Holmes, T., de Jeu, R., Naeimi, V., and Wagner, W., 2008: A possible solution for the problem of estimating the error structure of global soil moisture data sets, Geophys. Res. Lett., 35, L24403, doi: $10.1029 / 2008 \mathrm{gl035599.}$

Sellers, P. J., D. A. Randall, G. J. Collatz, J. A. Berry, C. B. Field, D. A. Dazlich, C. Zhang, G. D. Collelo, and L. Bounoua, 1996: A Revised Land Surface Parameterization (SiB2) for Atmospheric GCMS. Part I: Model Formulation. Journal of Climate, 9(4), 676-705.

Seneviratne, S. I., and R. Stöckli, 2008: The Role of Land-Atmosphere Interactions for Climate Variability in Europe, in Climate Variability and Extremes during the Past 100 Years, edited by S. Brönnimann, J. Luterbacher, T. Ewen, H. F. Diaz, R. S. Stolarski and U. Neu, pp. 179193, Springer Netherlands.

Seneviratne, S. I., P. Viterbo, D. Lüthi, and C. Schär, 2004: Inferring Changes in Terrestrial Water Storage Using ERA-40 Reanalysis Data: The Mississippi River Basin. Journal of Climate, 17, 2039-2057.

Seneviratne, S. I., T. Corti, E. L. Davin, M. Hirschi, E. B. Jaeger, I. Lehner, B. Orlowsky, and A. J. Teuling, 2010: Investigating soil moisture-climate 
interactions in a changing climate: A review. Earth-Science Reviews, 99 (3-4), 125-161.

Sheffield, J., G. Goteti, and E. F. Wood, 2006: Development of a 50-Year High-Resolution Global Dataset of Meteorological Forcings for Land Surface Modeling. Journal of Climate, 19, 3088-3111.

Siebert, S., P. Döll, J. Hoogeveen, J. M. Faures, K. Frenken, and S. Feick, 2005: Development and validation of the global map of irrigation areas. Hydrol. Earth Syst. Sci., 9(5), 535-547.

Siebert, S., V. Henrich, K. Frenken, and J. Burke, 2013: Global Map of Irrigation Areas version 5. Rheinische Friedrich-Wilhelms-University, Bonn, Germany / Food and Agriculture Organization of the United Nations, Rome, Italy.

Simmons, A., S. Uppala, D. Dee, and S. Kobayashi, 2006: ERA-Interim: New ECMWF reanalysis products from 1989 onwards, ECMWF Newsletter, 110, 26-35.

Simmons, A. J., K. M. Willett, P. D. Jones, P. W. Thorne, and D. P. Dee, 2010: Low-frequency variations in surface atmospheric humidity, temperature, and precipitation: Inferences from reanalyses and monthly gridded observational data sets, J. Geophys. Res., 115, D01110, doi:10.1029/2009jd012442.

Sneyers, R., 1975: Sur I'analyse statistique des séries d'observations, Secrétariat de I'Organisation Météorologique Mondiale, Genéve.

Su, Z., T. Schmugge, W. P. Kustas, and W. J. Massman, 2001: An Evaluation of Two Models for Estimation of the Roughness Height for Heat Transfer between the Land Surface and the Atmosphere. Journal of Applied Meteorology, 40, 1933-1951.

Su, Z., P. de Rosnay, J. Wen, L. Wang, and Y. Zeng, 2013: Evaluation of ECMWF's soil moisture analyses using observations on the Tibetan Plateau. Journal of Geophysical Research: Atmospheres, 118, 53045318.

Sun, J., 1999: Diurnal Variations of Thermal Roughness Height over a Grassland. Boundary-Layer Meteorology, 92, 407-427. 
Swenson, S., and J. Wahr, 2006: Post-processing removal of correlated errors in GRACE data. Geophysical Research Letters, 33, L08402.

Swenson, S. C., and P. C. D. Milly, 2006: Climate model biases in seasonality of continental water storage revealed by satellite gravimetry, Water Resour. Res., 42, W03201, doi:10.1029/2005WR004628.

Syed, T. H., J. S. Famiglietti, M. Rodell, J. Chen, and C. R. Wilson, 2008: Analysis of terrestrial water storage changes from GRACE and GLDAS. Water Resources Research, 44(2), W02433.

Tapley, B. D., S. Bettadpur, M. Watkins, and C. Reigber, 2004a: The gravity recovery and climate experiment: Mission overview and early results. Geophysical Research Letters, 31, L09607.

Tapley, B. D., S. Bettadpur, J. C. Ries, P. F. Thompson, and M. M. Watkins, 2004b: GRACE Measurements of Mass Variability in the Earth System. Science, 305(5683), 503-505.

Taylor, K. E., 2001: Summarizing multiple aspects of model performance in a single diagram. Journal of Geophysical Research: Atmospheres, 106, 7183-7192.

Teuling, A. J., et al., 2009: A regional perspective on trends in continental evaporation. Geophys. Res. Lett., 36, L02404, doi: 10.1029/2008GL036584.

Thomas, A., 2008: Agricultural irrigation demand under present and future climate scenarios in China. Global and Planetary Change, 60(3-4), 306326

van Beek, L. P. H., Y. Wada, and M. F. P. Bierkens, 2011: Global monthly water stress: 1 . Water balance and water availability. Water Resour. Res., 47, W07517, doi:10.1029/2010WR009791.

van den Hurk, B. J. J. M., P. Viterbo, A. C. M. Beljaars, and A. K. Betts, 2000: Offline validation of the ERA40 surface scheme, in ECMWF Tech.Memo 295, 43 pp., European Centre for Medium-Range Weather Forecasts, Reading, UK. (Available at http://www.ecmwf.int/publications/library/ecpublications/ pdf/tm/001-300/tm295.pdf) 
van der Velde, R., Z. Su, and Y. Ma, 2008: Impact of Soil Moisture Dynamics on ASAR $\sigma^{\circ}$ and Its Spatial Variability Observed over the Tibetan Plateau, Sensors, 8, 5479-5491.

van Loon, A. F., and H. A. J. van Lanen, 2013: Making the distinction between water scarcity and drought using an observation-modeling framework. Water Resour. Res., 49, 1483-1502, doi: 10.1002/wrcr.20147.

Viterbo, P., and A. C. M. Beljaars, 1995: An Improved Land Surface Parameterization Scheme in the ECMWF Model and Its Validation. Journal of Climate, 8(11), 2716-2748.

Vörösmarty, C. J., et al., 2010: Global threats to human water security and river biodiversity. Nature, 467(7315), 555-561.

Voss, K. A., J. S. Famiglietti, M. Lo, C. de Linage, M. Rodell, and S. C. Swenson, 2013: Groundwater depletion in the Middle East from GRACE with implications for transboundary water management in the TigrisEuphrates-Western Iran region. Water Resources Research, 49(2), 904914.

Wada, Y., D. Wisser, and M. F. P. Bierkens, 2014: Global modeling of withdrawal, allocation and consumptive use of surface water and groundwater resources. Earth Syst. Dynam., 5(1), 15-40.

Wada, Y., L. P. H. van Beek, C. M. van Kempen, J. W. T. M. Reckman, S. Vasak, and M. F. P. Bierkens, 2010: Global depletion of groundwater resources. Geophys. Res. Lett., 37, L20402, doi: 10.1029/2010GL044571.

Wada, Y., L. P. H. van Beek, and M. F. P. Bierkens, 2011a: Modelling global water stress of the recent past: on the relative importance of trends in water demand and climate variability. Hydrol. Earth Syst. Sci., 15(12), 3785-3808.

Wada, Y., L. P. H. van Beek, D. Viviroli, H. H. Dürr, R. Weingartner, and M. F. P. Bierkens, 2011b: Global monthly water stress: 2. Water demand and severity of water stress. Water Resour. Res., 47, W07518, doi: 10.1029/2010WR009792. 
Wagener, T., M. Sivapalan, P. A. Troch, B. L. McGlynn, C. J. Harman, H. V. Gupta, P. Kumar, P. S. C. Rao, N. B. Basu, and J. S. Wilson, 2010: The future of hydrology: An evolving science for a changing world, Water Resour. Res., 46(5), W05301, doi: 10.1029/2009WR008906.

Wang, J., Y. Sheng, C. J. Gleason, and Y. Wada, 2013: Downstream Yangtze River levels impacted by Three Gorges Dam. Environmental Research Letters, 8(4), 044012.

Wang, X., C. de Linage, J. Famiglietti, and C. S. Zender, 2011: Gravity Recovery and Climate Experiment (GRACE) detection of water storage changes in the Three Gorges Reservoir of China and comparison with in situ measurements. Water Resources Research, 47(12), W12502.

Wang, X.-P., R. Berndtsson, X.-R. Li, and E.-S. Kang, 2004: Water balance change for a re-vegetated xerophyte shrub area/Changement du bilan hydrique d'une zone replantée d'arbustes xérophiles. Hydrological Sciences Journal, 49(2), 283-295.

Wei, J., P. A. Dirmeyer, M. G. Bosilovich, and R. Wu, 2012: Water vapor sources for Yangtze River Valley rainfall: Climatology, variability, and implications for rainfall forecasting. Journal of Geophysical Research: Atmospheres, 117, D05126.

WHYMAP, 2008: World-wide Hydrogeological Mapping and Assessment Programme. Groundwater Resources of the World (1:25000000). BGR/UNESCO.

Winter, T. C., J. W. Harvey, O. L. Franke, and W. M. Alley, 1998: Ground Water and Surface Water A Single Resource. U.S. Geological Survey Circular 1139, Denver, Colorado

Wisser, D., S. Frolking, E. M. Douglas, B. M. Fekete, C. J. Vörösmarty, and A. H. Schumann, 2008: Global irrigation water demand: Variability and uncertainties arising from agricultural and climate data sets. Geophys. Res. Lett., 35, L24408, doi: 10.1029/2008GL035296.

Wu, L., Q. Zhang, and Z. Jiang, 2006: Three Gorges Dam affects regional precipitation. Geophysical Research Letters, 33, L13806. 
Xu, X., and Coauthors, 2008: A new integrated observational system over the Tibetan Plateau. Bull. Amer. Meteor. Soc., 89, 1492-1496, doi: 10.1175/2008BAMS2557.1.

Xue, B.-L., L. Wang, X. Li, K. Yang, D. Chen, and L. Sun, 2013: Evaluation of evapotranspiration estimates for two river basins on the Tibetan Plateau by a water balance method, Journal of Hydrology, 492(0), 290297.

Yan, H. M., X. Duan, and J. G. Cheng, 2007: Study on a severe drought event over Yunnan in spring 2005, J. Trop. Meteorol., 23, 300-306, 2007. (In Chinese)

Yanai, M., and G.-X. Wu, 2006: Effects of the Tibetan Plateau. The Asian Monsoon, Springer Berlin Heidelberg, 513-549.

Yanai, M., C. Li, and Z. Song, I992: Seasonal heating of the Tibetan Plateau and its effects on the evolution of the Asian summer monsoon, $J$. Meteor. Soc. Japan, 70, 319-351.

Yang, J., D. Gong, W. Wang, M. Hu, and R. Mao, 2012: Extreme drought event of 2009/2010 over southwestern China. Meteorol. Atmos. Phys., 115, 173-184.

Yang, K., Y. Y. Chen, and J. Qin, 2009: Some practical notes on the land surface modeling in the Tibetan Plateau. Hydrol. Earth Syst. Sci., 13, 687-701.

Yang, K., T. Koike, and D. Yang, 2003: Surface flux parameterization in the Tibetan Plateau. Bound.-Layer Meteor., 106, 245-262, doi: 10.1023/A:1021152407334.

Yang, K., T. Koike, and B. Ye, 2006: Improving estimation of hourly, daily, and monthly solar radiation by importing global data sets, Agricultural and Forest Meteorology, 137, 43-55.

Yang, K., T. Koike, B. Ye, and L. Bastidas, 2005: Inverse analysis of the role of soil vertical heterogeneity in controlling surface soil state and energy partition. Journal of Geophysical Research: Atmospheres, 110, D08101. 
Yang, K., M. Rasmy, S. Rauniyar et al., 2007: Initial CEOP-based review of prediction skill of operational general circulation models and land surface models, J. Appl. Meteorol. Climatol., 85A, 99-116.

Yang, K., and Coauthors, 2008: Turbulent flux transfer over bare-soil surfaces: Characteristics and parameterization. J. Appl. Meteor. Climatol., 47, 276-290, doi:10.1175/2007JAMC1547.1.

Yang, R., and M. A. Friedl, 2003: Modeling the effects of three-dimensional vegetation structure on surface radiation and energy balance in boreal forests. Journal of Geophysical Research: Atmospheres, 108 (D16), 8615.

Yang, S. L., J. Zhang, J. Zhu, J. P. Smith, S. B. Dai, A. Gao, and P. Li, 2005: Impact of dams on Yangtze River sediment supply to the sea and delta intertidal wetland response. Journal of Geophysical Research: Earth Surface, 110, F03006.

Yang, S. L., and Coauthors, 2010: Temporal variations in water resources in the Yangtze River (Changjiang) over the Industrial Period based on reconstruction of missing monthly discharges. Water Resources Research, 46, W10516.

Yang, Z.-L., and G.-Y. Niu, 2003: The Versatile Integrator of Surface and Atmosphere processes: Part 1. Model description. Global and Planetary Change, 38, 175-189.

Yang, Z.-L., and Coauthors, 2011: The community Noah land surface model with multiparameterization options (Noah-MP): 2. Evaluation over global river basins. Journal of Geophysical Research: Atmospheres, 116, D12110.

Yin, R., Q. Xiang, J. Xu, and X. Deng, 2010: Modeling the Driving Forces of the Land Use and Land Cover Changes Along the Upper Yangtze River of China. Environmental Management, 45, 454-465.

Zaitchik, B. F., A. K. Macalady, L. R. Bonneau, and R. B. Smith, 2006: Europe's 2003 heat wave: a satellite view of impacts and landatmosphere feedbacks. International Journal of Climatology, 26(6), 743-769. 
Zaitchik, B. F., M. Rodell, and F. Olivera, 2010: Evaluation of the Global Land Data Assimilation System using global river discharge data and a source-to-sink routing scheme. Water Resources Research, 46, W06507.

Zhang, L., W. R. Dawes, and G. R. Walker, 2001: Response of mean annual evapotranspiration to vegetation changes at catchment scale. Water Resources Research, 37, 701-708.

Zhang, J., L. Wu, and W. Dong, 2011: Land-atmosphere coupling and summer climate variability over East Asia. Journal of Geophysical Research: Atmospheres, 116, D05117.

Zhang, Z., B. F. Chao, J. Chen, and C. R. Wilson, 2015: Terrestrial water storage anomalies of Yangtze River Basin droughts observed by GRACE and connections with ENSO. Global and Planetary Change, 126(0), 3545.

Zheng, W., and Coauthors, 2012: Improvement of daytime land surface skin temperature over arid regions in the NCEP GFS model and its impact on satellite data assimilation. Journal of Geophysical Research: Atmospheres, 117, D06117.

Zheng, D., R. Van Der Velde, Z. Su, M. J. Booij, and A. Y. Hoekstra, 2014: Assessment of Roughness Length Schemes Implemented within the Noah Land Surface Model for High Altitude Regions, Journal of Hydrometeorology, 15, 921-937.

Zhu, Y., H. Wang, W. Zhou, and J. Ma, 2011: Recent changes in the summer precipitation pattern in East China and the background circulation. Clim Dyn, 36, 1463-1473.

Zhong, L., Y. Ma, M. S. Salama, and Z. Su, 2010: Assessment of vegetation dynamics and their response to variations in precipitation and temperature in the Tibetan Plateau. Climatic Change, 103, 519-535.

Zilitinkevich, S. S. 1995: Non-local turbulent transport: Pollution dispersion aspects of coherent structure of convective flows, in Air Pollution Theory and Simulation, vol. 1, Air Pollution III, edited by H. Power, N. Moussiopoulos, and C. A. Brebbia, pp. 53-60, Comput. Mech., Billerica, Mass. 
Zou, X. K. and H. Gao, 2007: Analysis of severe drought and heat wave over the Sichuan Basin in the summer of 2006, Adv. Clim. Chang. Res., 3, 149-153. (In Chinese) 


\section{Summary}

Climate variability and human activity have substantial effects on hydrological systems. Understanding and quantifying the hydrological responses to climate variability and human activity are increasingly important in water managements in river basins. The research described in this dissertation takes the Yangtze River basin as the study area and focuses on understanding and quantifying the spatial effects of climate variability and human activities on the terrestrial water storage (TWS) variation of the basin. This contributes to a better understanding of hydrologic processes and provides insight in separating natural and human effects on the hydrological systems in river basins.

This dissertation can be divided into two parts. The first part, Chapters 3 to 5 , is the preparatory part, with the aim to produce and/or obtain reliable TWS estimates for further investigation. In this part, the data assimilation products, the ERA-Interim Reanalysis Data (ERA-Interim) and Global Data Assimilation System (GLDAS) datasets, are assessed for the study area, the Noah and Noah-MP land surface models (LSMs) are used for hydro-meteorological simulations, and some model's parameterizations are adopted to improve the simulation accuracy. The first part contributes to the land surface modeling at a regional scale, especially for the Tibetan Plateau which is one of the most challenging regions for land surface modelling. The evaluation of the revised roughness lengths for regional scale land surface modelling of high-altitude catchment in Chapter 4 and the reconstructed water budget for the Yangtze River basin in Chapter 5 provide useful information for other modelers and hydrological data users.

The second part, Chapters 6 to 7, focuses on the ultimate goal of this dissertation and represents the main scientific contributions. Based on the first part, the ERAInterim produced TWS data are selected to investigate the impacts of climate variability on TWS variations in Chapter 6. In Chapter 7, besides the ERA-Interim data, a considerable amount of data from various sources including model results, remotely sensed data, and field measurements, are analyzed to investigate the 
human effects on spatiotemporal TWS variations. The results show that, mainly due to a decrease in the amount of precipitation, the period 2005-2010 was the driest period in terms of TWS in the basin, especially in the middle and lower Yangtze reaches since 1979. The natural part of TWS variation was 'stationary' during that dry period, but the human-induced TWS was continually increasing in the middle and south eastern parts of the basin, at a mean rate of about $3 \mathrm{~cm} \mathrm{yr}^{-1}$. The dominant contributor to the TWS excess was found to be intensive surface water irrigation, which recharged the water table in the middle and south eastern parts of the basin. Water impoundment of the Three Gorges Reservoir (TGR) was found to account for nearly $20 \%$ of the human-induced TWS increment in the region where the TGR is located. This attributed contribution of human-induced changes to the TWS is validated by quantifying the human impacts on surface water and groundwater storage changes based on combing field data with model simulation, and supported by additional data from various sources.

This $\mathrm{PhD}$ research deals with an important, interesting and challenging topic with very limited field data. Although the case study is the Yangtze River basin, the method employed in this research can be widely used in other river basins, and the framework for detection and attribution of TWS changes proposed in Chapter 7 is suggested as a viable way to separate natural and human effects on the hydrological system. 


\section{Samenvatting}

Klimaat-variabiliteit en menselijke activiteit hebben substantiële effecten op hydrologische systemen. Begrip en kwantificatie van de hydrologische respons op klimaat-variabiliteit en menselijke activiteit zijn van toenemend belang voor het waterbeheer in stroomgebieden. Het onderzoek in deze dissertatie gebruikt het stroomgebied van de Yangtze rivier als studiegebied en richt zich er op de ruimtelijke effecten van klimaat-variabiliteit en menselijke activiteit op variaties in de terrestrische watervoorraden in het stroomgebied (TWS) te begrijpen en kwantificeren. Dit draagt bij aan een beter begrip van hydrologische processen en geeft inzicht in het ontrafelen van natuurlijke en menselijke effecten op de hydrologische systemen in stroomgebieden.

Deze dissertatie bestaat uit twee delen. Het eerste deel, Hoofdstukken 3 tot 5 , is het voorbereidende deel dat tot doel heeft betrouwbare schattingen van TWS te produceren en/of te verkrijgen voor nader onderzoek. In dit deel worden dataassimilatie-producten, ERA-Interim Reanalysis Data (ERA-Interim) en Global Data Assimilation System (GLDAS) datasets, geëvalueerd voor het studiegebied; de Noah and Noah-MP landoppervlakte modellen (LSMs) worden gebruikt voor hydro-meteorologische simulaties en enkele modelparameterisaties worden aangepast om de nauwkeurigheid van simulaties te verbeteren. Het eerste deel draagt bij aan de landoppervlakte modellering op een regionale schaal, in het bijzonder voor het Tibetaans Plateau, een van de meest uitdagende gebieden voor de landoppervlakte modellering. De evaluatie van de aangepaste ruwheidslengtes voor regionale landoppervlakte modellering van de hoogvlakte in Hoofdstuk 4 en de reconstructie van de waterbalans voor het stroomgebied van de Yangtze in Hoofdstuk 5 verschaffen nuttige informatie voor andere modelleurs en gebruikers van hydrologische data.

Het tweede deel, Hoofdstukken 6 en 7, gaat in op het uiteindelijke doel van de dissertatie en presenteert de belangrijkste wetenschappelijke bijdrages. Op basis van het eerste deel zijn TWS data, geproduceerd met ERA-Interim, geselecteerd om de gevolgen van klimaat-variabiliteit op TWS variaties te onderzoeken in Hoofdstuk 6. In hoofdstuk 7 wordt, naast de ERA-Interim data, een aanzienlijke 
hoeveelheid data geanalyseerd uit verschillende bronnen, waaronder model resultaten, satellietwaarnemingen en veldmetingen, om de menselijke beïnvloeding van TWS variaties in ruimte en tijd te onderzoeken. Resultaten laten zien dat de periode 2005-2010 de droogste periode was sinds 1979 in termen van TWS in het stroomgebied, met name in de middenstroom en benedenstroom van de Yangtze, vooral veroorzaakt door een afname in de neerslaghoeveelheid. Het natuurlijke deel van de variaties in TWS was gedurende die droge periode 'stationair', maar de menselijk-beïnvloede TWS was continu stijgend in de centrale en zuidoostelijke delen van het stroomgebied, met een gemiddelde toename van ongeveer $3 \mathrm{~cm} \mathrm{yr}^{-1}$. De intensieve irrigatie uit oppervlaktewater, die het grondwater aanvulde in de centrale en zuidoostelijke delen van het stroomgebied, kan aangewezen worden als dominante bijdrage aan de verhoogde TWS. Wateropslag in de Three Gorges Reservoir (TGR) was verantwoordelijk voor bijna $20 \%$ van de menselijk-beïnvloede toename van TWS in de omgeving van de TGR. Deze identificatie van de menselijk-beïnvloede bijdrage aan de TWS wordt gevalideerd door de gevolgen van menselijke activiteiten voor de voorraden oppervlakte- en grondwater te kwantificeren op basis van een combinatie van veldwaarnemingen, model simulaties en ondersteund door additionele data uit verscheidene bronnen.

Dit PhD onderzoek behandelt een belangrijk, interessant en uitdagend onderwerp met zeer beperkte veldwaarnemingen. Hoewel het Yangtze stroomgebied is gebruikt als studiegebied zijn de gebruikte onderzoeksmethoden breed inzetbaar in andere stroomgebieden, en kan het voorgestelde raamwerk voor de detectie en toerekening van TWS veranderingen uit Hoofdstuk 7 een waardevolle manier zijn om natuurlijke en menselijke effecten op het hydrologische systeem te ontrafelen. 


\section{About the author}

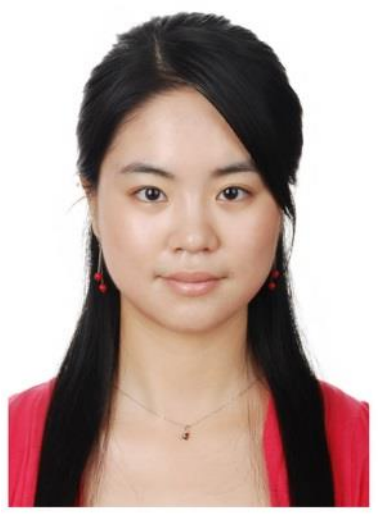

Ying Huang was born in Fenyi, Jiangxi Province, China on 05 November, 1985. She was studying in secondary and high schools in Fenyi, Xinyu during the period 1997-2003.

In September 2003, she joined Taiyuan University of Technology in Taiyuan, China, and graduated in 2007 with a Bachelor of Engineering degree on Urban and Rural Planning \& Resources Management.

Later in 2007, she became a M.Sc. student of physical geography at the State Key Laboratory of Estuarine and Coastal Research (SKLEC), East China Normal University in Shanghai, China. Two years later, she was directly promoted by SKLEC to be a PhD student.

In October 2010, she went to the Netherlands for her PhD research on the project "hydrological responses to climate variability and human activity in the Yangtze River basin". This is a jointed PhD project between Water Resource department of Faculty of Geo-Information Science and Earth Observation (ITC) and Water Engineering and Management department (WEM) of Faculty of Engineering Technology at the University of Twente in the Netherlands.

In February 2016, she will start working as a researcher at SKLEC in Shanghai, China. 
